\title{
The metal content of bulge field stars from FLAMES-GIRAFFE spectra
}

\section{Stellar parameters and iron abundances ${ }^{\star} \star \star \star$}

\author{
M. Zoccali ${ }^{1}$, V. Hill ${ }^{2}$, A. Lecureur ${ }^{2,3}$, B. Barbuy ${ }^{4}$, A. Renzini ${ }^{5}$, D. Minniti ${ }^{1}$, A. Gómez $^{2}$, and S. Ortolani ${ }^{6}$ \\ 1 P. Universidad Católica de Chile, Departamento de Astronomía y Astrofísica, Casilla 306, Santiago 22, Chile \\ e-mail: [mzoccali; dante]@astro.puc.cl \\ 2 GEPI, Observatoire de Paris, CNRS, Université Paris Diderot; Place Jules Janssen, 92190 Meudon, France \\ e-mail: [Vanessa.Hill; Aurelie.Lecureur; Ana.Gomez]@obspm.fr \\ 3 Astronomisches Rechen-Institut, Zentrum für Astronomie der Universität Heidelberg, Mönchhofstr. 12-14, 69120 Heidelberg, \\ Germany \\ 4 Universidade de São Paulo, IAG, Rua do Matão 1226, Cidade Universitária, São Paulo 05508-900, Brazil \\ e-mail: barbuy@astro.iag.usp.br \\ 5 INAF - Osservatorio Astronomico di Padova, Vicolo dell'Osservatorio 2, 35122 Padova, Italy \\ e-mail: alvio.renzini@oapd.inaf.it \\ ${ }^{6}$ Università di Padova, Dipartimento di Astronomia, Vicolo dell'Osservatorio 5, 35122 Padova, Italy \\ e-mail: sergio.ortolani@unipd.it
}

Received 14 January 2008 / Accepted 26 April 2008

\section{ABSTRACT}

\begin{abstract}
Aims. We determine the iron distribution function (IDF) for bulge field stars, in three different fields along the Galactic minor axis and at latitudes $b=-4^{\circ}, b=-6^{\circ}$, and $b=-12^{\circ}$. A fourth field including NGC 6553 is also included in the discussion.

Methods. About 800 bulge field K giants were observed with the GIRAFFE spectrograph of FLAMES@VLT at spectral resolution $R \sim 20000$. Several of them were observed again with UVES at $R \sim 45000$ to insure the accuracy of the measurements. The LTE abundance analysis yielded stellar parameters and iron abundances that allowed us to construct an IDF for the bulge that, for the first time, is based on high-resolution spectroscopy for each individual star.

Results. The IDF derived here is centered on solar metallicity, and extends from $[\mathrm{Fe} / \mathrm{H}] \sim-1.5$ to $[\mathrm{Fe} / \mathrm{H}] \sim+0.5$. The distribution is asymmetric, with a sharper cutoff on the high-metallicity side, and it is narrower than previously measured. A variation in the mean metallicity along the bulge minor axis is clearly between $b=-4^{\circ}$ and $b=-6^{\circ}([\mathrm{Fe} / \mathrm{H}]$ decreasing $\sim$ by 0.6 dex per kpc). The field at $b=-12^{\circ}$ is consistent with the presence of a gradient, but its quantification is complicated by the higher disk/bulge fraction in this field.

Conclusions. Our findings support a scenario in which both infall and outflow were important during the bulge formation, and then suggest the presence of a radial gradient, which poses some challenges to the scenario in which the bulge would result solely from the vertical heating of the bar.
\end{abstract}

Key words. Galaxy: bulge - stars: abundances - stars: atmospheres

\section{Introduction}

The Galactic bulge is the nearest galactic spheroid, and it can be studied in greater detail than any other one. In particular, its stellar content can be characterized in terms of age and composition distribution functions, coupled with kinematical information. Thus, the bulge offers a unique opportunity to construct the star formation and mass assembly history of a galactic spheroid, hence providing a unique benchmark for theories of galaxy formation. The Galactic bulge is dominated by stellar populations older than 10 Gyr (Ortolani et al. 1995; Feltzing \& Gilmore 2000; Zoccali et al. 2003), with no detectable trace of younger stellar populations. As a result, most of its stars were formed at a cosmic epoch that corresponds to $z \gtrsim 2$, making its study quite complementary to that of galaxies at such high redshifts.

\footnotetext{
* Observations collected at the European Southern Observatory, Paranal, Chile (ESO programmes 71.B-0617 and 73.B-0074).

$\star \star$ Full Table 3 is only available in electronic form at

http://www . aanda.org
}

Starting with the pioneering spectroscopic study of Rich (1988), the distribution function of the iron abundance among bulge stars has been further explored and refined by McWilliam \& Rich (1994), Ibata \& Gilmore (1995a,b) Minniti (1996), Sadler et al. (1996), Ramírez et al. (2000), and Fulbright et al. (2006), using spectroscopic observations, and by Zoccali et al. (2003) with a purely photometric method. Among them, the McWilliam \& Rich (1994) and Fulbright et al. (2006) analyses deserve special mention because they were the only ones to obtain high-resolution spectra, although only for a small sample of stars (11 and 27, respectively), used to calibrate some previous, low-resolution analysis of a larger sample. The choice of this method was dictated by high resolution spectroscopic surveys being carried out with long-slit spectrographs, thus observing just one or two stars at a time. With the advent of the FLAMES multiobject spectroscopic facility at the VLT (Pasquini et al. 2003) it then became possible to observe a large number of objects simultaneously, at high spectral resolution, a quantum jump in this kind of studies. 
Table 1. Characteristics of the four bulge fields.

\begin{tabular}{llccrcc}
\hline \hline Nr. & Identification & $l$ & $b$ & $\begin{array}{r}R_{\mathrm{GC}} \\
\mathrm{pc})\end{array}$ & $E(B-V)$ & $N_{\text {stars }}$ \\
\hline 1 & Baade's Window & 1.14 & -4.18 & 604 & 0.55 & 204 \\
2 & $b=-6^{\circ}$ Field & 0.21 & -6.02 & 850 & 0.48 & 213 \\
3 & $b=-12^{\circ}$ Field & 0.00 & -12.0 & 1663 & 0.20 & 104 \\
4 & NGC 6553 Field & 5.25 & -3.02 & 844 & 0.70 & 201 \\
\hline
\end{tabular}

Table 2. Magnitude, color and $S / N$ range of the spectroscopic targets.

\begin{tabular}{llccc}
\hline \hline Nr. & Identification & $\begin{array}{c}(V-I) \\
\text { range }\end{array}$ & $\begin{array}{c}I \\
\text { range }\end{array}$ & $\begin{array}{c}\text { typical } S / N \\
\text { @ 6200 }\end{array}$ \\
\hline 1 & Baade's Window & $1.53-2.62$ & $14.20-14.70$ & $40-60$ \\
2 & $b=-6^{\circ}$ Field & $1.41-2.43$ & $14.00-14.50$ & $60-90$ \\
3 & $b=-12^{\circ}$ Field & $1.10-1.70$ & $13.70-14.93$ & $40-80$ \\
4 & NGC 6553 Field & $2.00-3.00$ & $14.00-14.50$ & $30-60$ \\
\hline
\end{tabular}

FLAMES feeds 8 fibers to the UVES high resolution spectrograph, and over 130 fibres to the GIRAFFE medium-high resolution spectrograph. The results of bulge stars observations of $50 \mathrm{~K}$ giants obtained with UVES with $R \simeq 45000$ have been reported by Zoccali et al. (2006) concerning the oxygen abundance and the $[\mathrm{O} / \mathrm{Fe}]$ vs. $[\mathrm{Fe} / \mathrm{H}]$ correlation, and by Lecureur et al. (2007) concerning the abundance of $\mathrm{O}, \mathrm{Na}, \mathrm{Mg}$, and $\mathrm{Al}$.

This paper is the first of a series devoted to the detailed chemical analysis of a sample of 720 bulge giant stars, in four different fields, observed with FLAMES-GIRAFFE with a resolution $R \sim 20000$. Another 220 bulge red clump stars were observed, in the same condition, as part of the GIRAFFE GTO programme (Lecureur et al. 2008). The latter sample is occasionally combined with the present one, in order to investigate some of the systematics and increase the statistics. Taking advantage of the FLAMES link to the UVES spectrograph, 58 target stars were also observed at higher spectral resolution $(R=45000)$, making it possible to compare abundances derived from medium and high resolution spectra.

\section{Observations}

Spectra for a sample of $\mathrm{K}$ giants in four bulge fields have been collected at the VLT-UT2 with the FLAMES-GIRAFFE spectrograph, at resolution $R \sim 20000$. A total wavelength range of $\sim 760 \AA$ has been covered through the setup combinations HR 13+HR 14+HR 15 (programme 071.B-0617) for fields 1 and 2 in Table 1, and HR 11+HR 13+HR 15 (programme 073.B-0074) for fields 3 and 4. The characteristics of the observed fields, together with the number of target stars contained in each, are listed in Table 1. The total exposure time varies from about $1 \mathrm{~h}$ to almost $5 \mathrm{~h}$, depending on the setup and on the star luminosity (targets have been divided into a bright and a faint group) in order to insure that the final $S / N$ of each coadded spectrum is $\sim 60$. In fact, the actual $S / N$ is not identical among the targets of a given field (see Table 2) due to the differences both in magnitude and in the average accuracy of fibre positioning.

\subsection{Photometric data}

In the color magnitude diagram, these stars are located on the red giant branch (RGB), roughly 1 mag above the red clump (see Table 2), as shown in the lower panels of Fig. 1. The astrometry and the photometric $V, I$ data come from the OGLE catalogue
(Udalski et al. 2002) for our Field-1, from archive WFI images obtained within the ESO Pre-FLAMES survey (e.g., Momany et al. 2001) from which our group obtained the stellar catalogue (Field-2 and -3), and from archive WFI images from proposal 69.D-0582A kindly reduced by Yazan Momany, for Field-4. Cross-identification with the $2 \mathrm{MASS}$ point source catalogue (Carpenter et al. 2001) allowed us to obtain $V, I, J, H, K$ magnitudes for each of the target stars. Some of the fields contain a globular cluster, namely NGC 6528 and NGC 6522 in Baade's Window, NGC 6558 in the $b=-6^{\circ}$ field, and NGC 6553 in the eponymous field. Member stars of these clusters will be discussed only marginally here, because they are the subject of dedicated papers (see Barbuy et al. 2007, for NGC 6558).

In order to avoid strong biases in the resulting iron distribution function (IDF) we included targets spanning the whole color range of the RGB at that magnitude. However, the need to maximize the number of targets while avoiding forbidden fibre crossings, makes it impossible to actually fine tune a uniform sampling of the RGB color span. The upper panels of Fig. 1 show as a thick histogram the ratio of the number of targets (dotted histogram) at each color bin, to the available stars (solid histogram). The scale of the thick histogram is shown on the right side of the figure. Since we expect a correlation between the RGB color and the metallicity of the stars, the ideal, unbiased sample would be one with a flat ratio between observed and available stars at each color ${ }^{1}$. As stated above, it is virtually impossible to keep this constraint all the way through the fibre allocation process. For this reason, further below in our analysis we will correct the raw IDF for this bias. We will do that in two independent ways, namely: $i$ ) we randomly remove from the IDF stars belonging to the most populated color bins, until we reach a flat target/available-star ratio; or ii) in the less populated color bins we duplicate some randomly-extracted stars, until we reach a flat target/available-star ratio. In both cases we will repeat the process 200 times, in order to minimize stochastic fluctuations in the final star list due to the random extractions, and we combine the results just by merging the 200 star lists. The resulting IDF from the two methods described above are indistinguishable, proving that the method is indeed robust (see Fig. 7 below).

The $V-I$ color was used to obtain photometric temperatures, according to the latest empirical calibration (Ramírez \& Meléndez 2005) based on the InfraRed Flux Method. As an additional indicator of the star temperature we measured the strength of the TiO band using an index defined between 6190-6250 $\AA$ (band) and 6120-6155 $\AA$ (continuum region). The strength of this index indeed correlates very well with the photometric temperatures, for $T_{\text {phot }}<4500 \mathrm{~K}$, where the TiO band is strong enough to be measured. The $\mathrm{TiO}$ index was used in two ways. First, it allowed us to establish that the $V-I$ color was the best one to derive photometric temperatures, as the one showing the smallest dispersion in the correlation between $T_{V-I}$ and $T_{\mathrm{TiO}}$. Second, since we expect that the $V-I$ color is more sensitive to differential reddening variations than the $\mathrm{TiO}$ index, for stars cooler than $4500 \mathrm{~K}$, we used the latter to estimate a $(V-I)_{0}$ color, to be converted into a photometric temperature. The calibration we used to convert the $\mathrm{TiO}$ index into a $(V-I)_{0}$ color was estimated as a linear fit to the observed correlation between the strength of the index and the $(V-I)_{0}$ obtained assuming a constant reddening for each field (see Table 1). Therefore, the use of the $T_{\mathrm{TiO}}$ for the coolest stars only minimizes the effect of the differential reddening. Any problem related to the adopted

\footnotetext{
1 Note that a region within 2 arcmin from the cluster center was removed both from the available-star and from the target sample.
} 


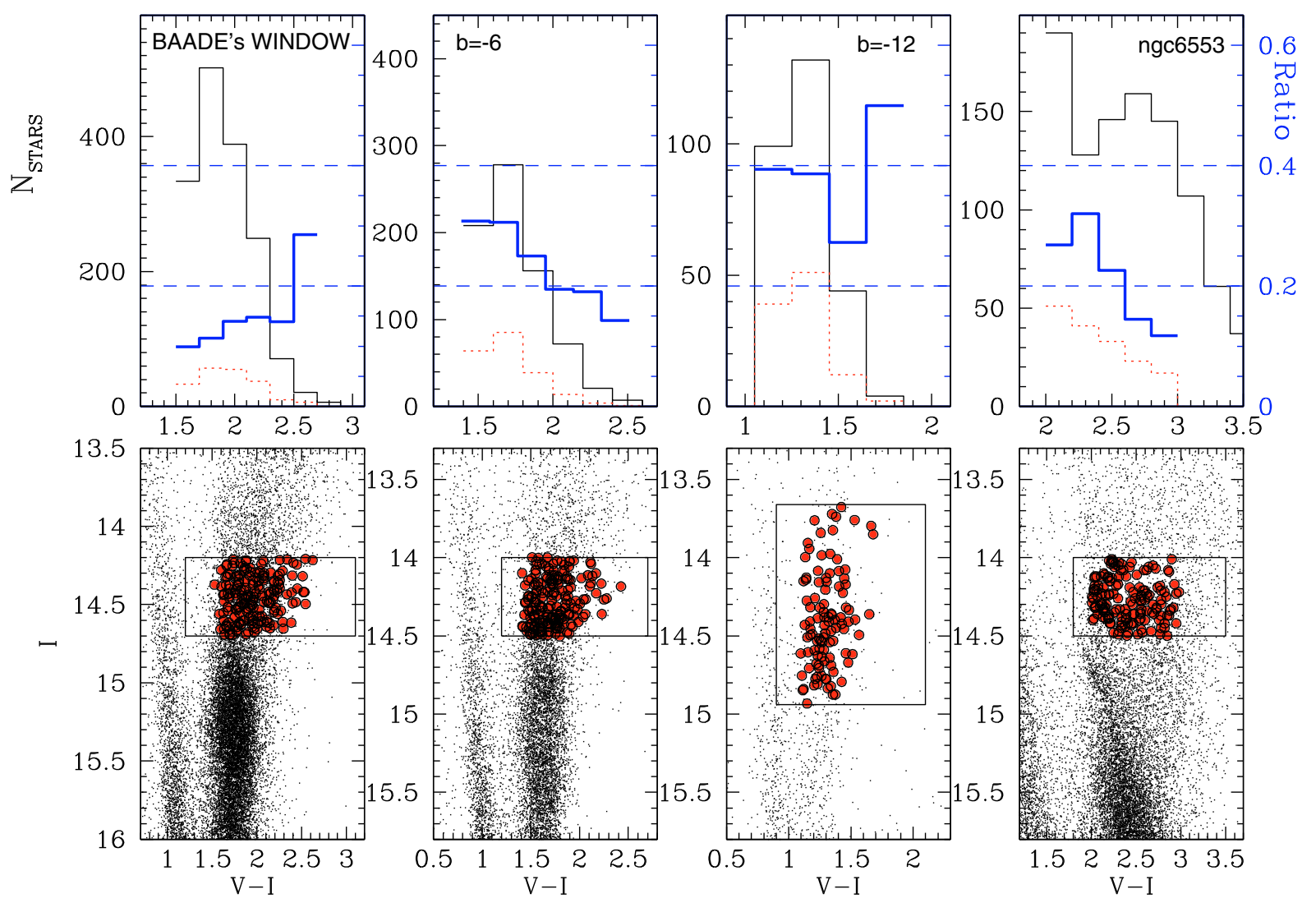

Fig. 1. Lower panels: the color magnitude diagram of the four observed fields, with the spectroscopic target stars marked as large filled circles. From left to right the fields are: Baade's Window, the $b=-6^{\circ}$ field, the $b=-12^{\circ}$ field and the NGC 6553 field. The large box shows the magnitude limits used for target selection, and the color limits of the upper panels. Upper panels: color histogram of the available stars in the selected target box (thin solid), of the actually observed targets (dotted) and of the ratio of observed to available stars (thick solid). The vertical scale of the latter histogram is shown on the right hand side of the upper panels, and two dashed lines have been drawn at 0.2 and 0.4 to help the eye in reading the figure.

color-temperature calibration by Ramírez \& Meléndez (2005) will obviously be present also in our TiO- $(V-I)_{\mathrm{O}}$-temperature calibration. Finally, it is worth emphasizing that the photometric temperature has only been used as an initial first guess. The final adopted temperature is the spectroscopic one, derived imposing excitation equilibrium on a sample of $\sim 60 \mathrm{FeI}$ lines.

Photometric gravity was instead calculated from the classical relation:

$\log g_{*}=\log g_{\odot}+4 \log \frac{T_{*}}{T_{\odot}}+0.4\left(M_{\mathrm{bol}}-M_{\mathrm{bol}, \odot}\right)+\log \frac{M_{*}}{M_{\odot}}$

adopting a mean distance of $8 \mathrm{kpc}$ for the bulge, $T_{\odot}=5770 \mathrm{~K}$, $\log g_{\odot}=4.44, M_{\text {bol } \odot}=4.75$ and $M_{*}=0.85 M_{\odot}$. Note that, at each step of the iterative process to converge on the stellar parameters and metallicity, described below, the photometric gravity was re-calculated using the appropriate (now spectroscopic) temperature and metallicity (to compute the $V$-band bolometric correction, from Alonso et al. 1999) for the star under analysis.

\subsection{Spectroscopic data}

Individual spectra were reduced with the GIRBLDRS pipeline ${ }^{2}$ provided by the FLAMES consortium (Geneva Observatory; Blecha et al. 2003), including bias, flatfield, extraction and wavelength calibration. All the spectra for each star (a number between 1 and 5, depending on the field) were then registered in

\footnotetext{
${ }^{2}$ Available at SouceForge, http://girbldrs. sourceforge . net
}

wavelength to correct for heliocentric radial velocity and coadded to a single spectrum per setup, per star. In each plate, about 20 GIRAFFE fibres were allocated to empty sky regions. These sky spectra were visually inspected to reject the few that might have evident stellar flux, and then coadded to a single sky spectrum. The latter was then subtracted from the spectrum of each target star. The equivalent widths (EWs) for selected iron lines were measured using the automatic code DAOSPEC (Stetson \& Pancino, in preparation ${ }^{3}$ ).

\section{Line list}

The selection of a clean line list, and the compilation of their atomic parameters, has been done with special care. An initial line list was compiled from NIST (Fuhr \& Wiese 2006). Each line was then checked against blends, in the relevant metallicity and temperature range, using synthetic spectra generated with and without the line, using the codes by Alvarez \& Plez (1998) and Barbuy et al. (2003). The oscillator strengths log gf of each of the clean lines were then modified by requiring that it would give $[\mathrm{Fe} / \mathrm{H}]=+0.30$ on the spectrum of $\mu$ Leonis, observed at the Canada-France-Hawaii Telescope with the ESPaDOnS spectrograph, at resolution $R=80000$ and $S / N \sim 500$. The following parameters were determined for $\mu$ Leo: $T_{\text {eff }}=4550 \mathrm{~K}$, $\log g=2.3$, microturbulence velocity $V_{\mathrm{t}}=1.3 \mathrm{~km} \mathrm{~s}^{-1}$. The final line list is thus the same used in Lecureur et al. (2007) with the

\footnotetext{
${ }^{3}$ http://cadcwww.hia.nrc.ca/stetson/daospec/
} 

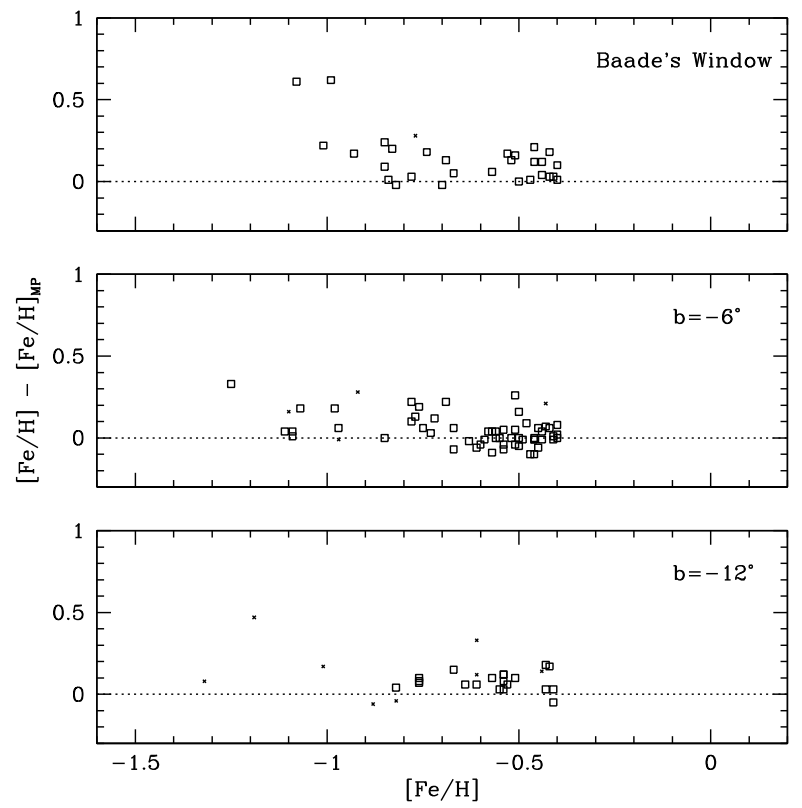

Fig. 2. Comparison between the iron abundance obtained using either the metalpoor or the metalrich line list, for stars with $[\mathrm{Fe} / \mathrm{H}]<-0.4$. Small symbols are stars for which the metal-rich line list was giving very poor results (e.g., it was not possible to converge on excitation equilibrium, or the dispersion of measurements from individual lines was too high) and those stars were thus marked as "low quality".

addition of some more lines in the region covered by the HR 11 GIRAFFE setup (5597-5728 $\AA$ ) not included in that paper. With the same set of atomic lines we obtained $[\mathrm{Fe} / \mathrm{H}]=-0.52$ for Arcturus $\left(T_{\text {eff }}=4300 \mathrm{~K}, \log g=2.5\right.$ and $\left.V_{\mathrm{t}}=1.5 \mathrm{~km} \mathrm{~s}^{-1}\right)$, and $[\mathrm{Fe} / \mathrm{H}]=-0.02$ for the Sun $\left(T_{\mathrm{eff}}=5777 \mathrm{~K}, \log g=4.4\right.$ and $\left.V_{\mathrm{t}}=1.0 \mathrm{~km} \mathrm{~s}^{-1}\right)$. The damping constants, taken from Coelho et al. (2005) were computed where possible, and in particular for most of the FeI lines, using the collisional broadening theory (Barklem et al. 1998, 2000).

This line list proved effective down to $[\mathrm{Fe} / \mathrm{H}] \sim-0.8$, including lines with a wide range of EWs for all the stars. For more metal-poor stars, however, we started lacking strong lines. A different line list was then compiled, including lines that would be too strong in $\mu$ Leo, but of intermediate strength in relatively metal poor stars. This one was produced with the same criterion described below, except that we have kept the NIST $\log g f \mathrm{~s}$. In order to ensure a smooth transition between the so called metalrich and the metalpoor line list, we measured the metallicity of Arcturus, from a UVES spectrum (Bagnulo et al. 2003) with both, and switch from one to the other at $[\mathrm{Fe} / \mathrm{H}]=-0.4$, where we check that the two would give consistent results. The metallicity of Arcturus with the metalpoor line list, and the same parameters listed above, is $[\mathrm{Fe} / \mathrm{H}]=-0.55$.

Figure 2 shows the difference between the $[\mathrm{Fe} / \mathrm{H}]$ values resulting from the use of the metalrich or the metalpoor line list, for stars with $[\mathrm{Fe} / \mathrm{H}]<-0.4$. It can be seen that at the transition region $(-0.6<[\mathrm{Fe} / \mathrm{H}]<-0.4)$ the systematic difference is 0.09 dex, 0.01 dex and 0.08 dex, for Baade's Window, the $b=-6^{\circ}$ field and the $b=-12^{\circ}$ field, respectively. For more metal poor stars the difference obviously increases, because the metalrich line list is clearly not appropriate for them.

Finally, in order to complete the analysis of possible systematics due to the adopted line list, we measured the metallicity of Arcturus by selecting only the wavelength ranges of the two setup combinations we used for our targets, namely
HR $13+$ HR $14+$ HR 15 or HR $11+$ HR $13+$ HR 15. The difference in $[\mathrm{Fe} / \mathrm{H}]$ is $+0.01 \mathrm{dex}$, the first setup combination giving higher metallicity, both with the metalpoor and with the metalrich line list. The value quoted above for the metallicity of Arcturus $([\mathrm{Fe} / \mathrm{H}]=-0.52)$ refers to the HR $13+\mathrm{HR} 14+\mathrm{HR} 15$ setup combination.

\section{Abundance analysis}

LTE abundance analysis was performed using well tested procedures (Spite 1967) and the new MARCS spherical models (Gustafsson et al. 2003; available at http://wwW. marcs.astro.uu.se/). Excitation equilibrium was imposed on FeI lines in order to refine the photometric $T_{\text {eff }}$, while photometric gravity was imposed even if ionization equilibrium was not fulfilled (cf. Zoccali et al. 2006). The microturbulence velocity $\left(V_{\mathrm{t}}\right)$ was found by imposing a constant $[\mathrm{Fe} / \mathrm{H}]$ for lines of different expected strengths (predicted EWs for a given stellar model). The reason for the latter choice is that, when plotting derived metallicities versus observed EWs, the obvious correlation of the errors (a too high $\mathrm{EW}$ would give a too high $[\mathrm{Fe} / \mathrm{H}]$, and vice versa) would lead us to detect a positive slope, hence to increase the $V_{\mathrm{t}}$ (Magain 1984). The effect may be negligible with very high $S / N$, high resolution spectra (i.e., when the errors on the EWs are also negligible) but we verified that it would introduce a significant systematic error in the measurements of the present GIRAFFE spectra. Extensive discussion of this effect can be found in Lecureur et al. (2008).

Finally, once converged on the best stellar parameters, we calculate the $[\mathrm{Fe} / \mathrm{H}]$ of each star as a weighted mean of the line-by-line measurements. The weight associated to each line is given by the inverse square of its abundance error, as derived from the error in the measured EWs.

\section{Estimates of metallicity uncertainties}

In this section we discuss all the available information about the error associated to the $[\mathrm{Fe} / \mathrm{H}]$ of each star. Some of our tests will quantify only the statistical errors, some others will quantify a combination of part of the systematics and the statistical errors. Finally, we will try to combine all this information together in order to estimate how far we can go in the interpretation of apparent features of the obtained IDFs.

\subsection{Line-to-line dispersion}

Figure 3 shows the scatter in the line-by-line $[\mathrm{Fe} / \mathrm{H}]$ measurement, divided by the square root of the number of lines, versus $[\mathrm{Fe} / \mathrm{H}]$. This is a fairly reasonable estimate of the statistical (line-to-line only) fluctuation associated with each $[\mathrm{Fe} / \mathrm{H}]$ value. Clearly, the more metal rich the star, the more crowded is the spectrum, hence the higher the dispersion of $[\mathrm{Fe} / \mathrm{H}]$ from individual lines. Baade's Window's stars show the largest scatter, likely due to the lower $S / N$ of those spectra, caused by the lower accuracy of the astrometry (from the OGLE catalogue) used to position the fibres. In any case, the statistical error from the dispersion of individual lines is less than 0.06 dex for Baade's Window, and less than 0.04 dex for the other fields.

\subsection{Degeneracy in the stellar parameters}

Because of a mistake in the fibre allocation, in the $b=-12^{\circ}$ field a sample of $\sim 100$ stars was observed twice, instead of switching 


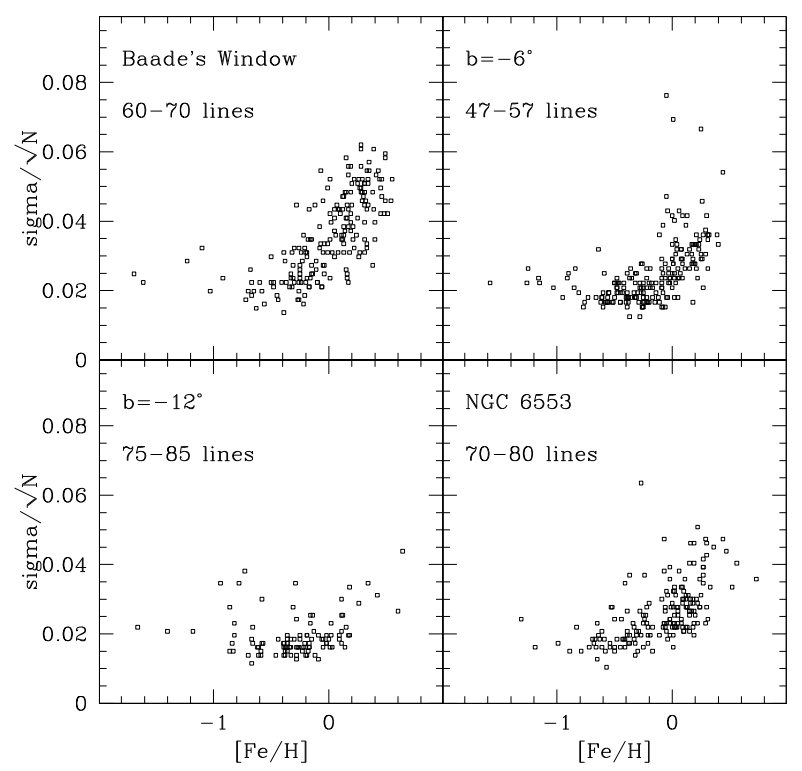

Fig. 3. The error on the mean $[\mathrm{Fe} / \mathrm{H}]$ of each star, due to the line-to-line dispersion. The minimum and maximum number of FeI lines found in each star is indicated in the figure labels.

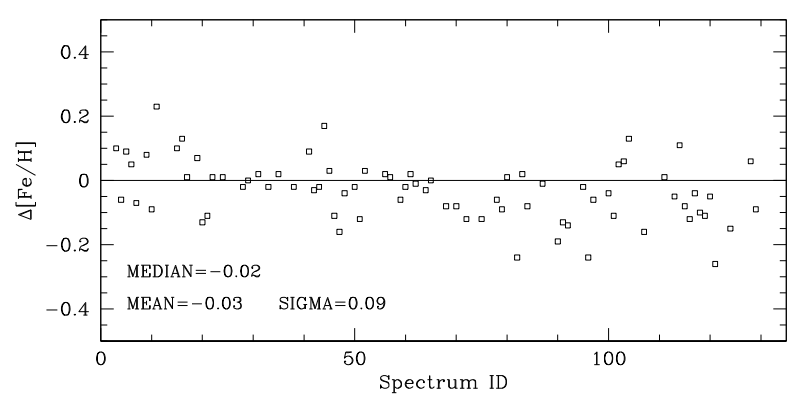

Fig. 4. The difference in $[\mathrm{Fe} / \mathrm{H}]$ between the two independent measurements of the two repeated sets of spectra in the $b=-12^{\circ}$ field.

to the next target sample. This unintentional duplication, on the other hand, turned out to be very useful to estimate the repeatability of our measurements. The two sets of spectra for the same stars have been reduced in a fully independent way, as if they were different stars, and the stellar parameters were also derived independently. Thus, the differences in the metallicity obtained for the same star is not only due to statistical fluctuations, but also to possible differences in the adopted parameters. Figure 4 shows the difference in the $[\mathrm{Fe} / \mathrm{H}]$ of each star, from the two sets of observations. The figure label shows the mean, median, and spread of the distribution. We checked for correlations of the $\Delta[\mathrm{Fe} / \mathrm{H}]$ against any stellar parameter $\left(T_{\mathrm{eff}}, V_{\mathrm{t}}\right.$, $[\mathrm{Fe} / \mathrm{H}], S / N, \ldots)$ but to our great surprise, the only quantity that showed a mild correlation with the $[\mathrm{Fe} / \mathrm{H}]$ difference is the spectrum ID, meaning the fibre position along the slit. Specifically, more than a trend what we see is a systematic offset between the first $\sim 65$ stars $(\langle\Delta[\mathrm{Fe} / \mathrm{H}]\rangle \approx 0)$ and the next ones $(\langle\Delta[\mathrm{Fe} / \mathrm{H}]\rangle \approx$ $-0.07)$. No physical property of the star is associated with this parameter, and it is unlikely that any instrumental effect could explain this behaviour. The difference is instead due to the $f u c$ tuations in the subjective process of converging to the best stellar parameters. In other words, since the stellar parameters, and in particular the temperature and the microturbulence velocity, produce similar results on the line-by-line $[\mathrm{Fe} / \mathrm{H}]$ abundances, it is possible to converge on two different model atmospheres

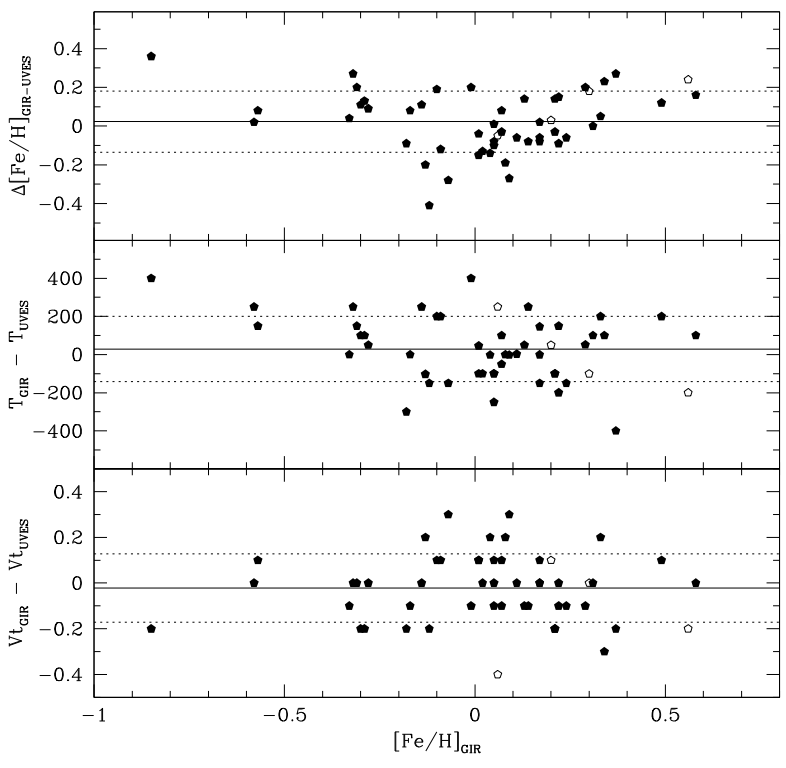

Fig. 5. Comparison between the measured iron abundances, temperature and microturbulence velocity in the stars observed both with UVES and GIRAFFE. Open symbols are stars with larger dispersion in the line-by-line iron measurements (mostly metal rich stars). The mean systematic difference (solid line) and the $\pm 1 \sigma$ spread around it (dotted lines) are shown.

(i.e., with both different $T_{\text {eff }}$ and different $V_{\mathrm{t}}$, compensating each other) while preserving both the excitation equilibrium and a constant abundance with EWs. The two models will give slightly different mean iron abundance. Therefore, the resulting $[\mathrm{Fe} / \mathrm{H}]$ may differ by as much as $\approx 0.07 \mathrm{dex}$, depending on whether one starts by iterating on $T_{\text {eff }}$ until reaching excitation equilibrium, then fixing the required $V_{\mathrm{t}}$, or one proceeds in the opposite direction, first fixing $V_{\mathrm{t}}$, and then iterating on $T_{\text {eff }}$. According to our records, this change of procedure occurred in fact around spectrum Nr. 65. While we could have re-analyzed the stars keeping a uniform procedure, we preferred to leave track of the effect that such difference in the analysis has caused on the resulting $[\mathrm{Fe} / \mathrm{H}]$. Hence, $\Delta[\mathrm{Fe} / \mathrm{H}]<0.07$ dex, is a good estimate of the mean fluctuations due to the subjective part of the analysis.

On the other hand, for stars with metallicity close to solar, a systematic error of $\pm 200 \mathrm{~K}$ in the adopted $T_{\text {eff }}$ (and corresponding change in the gravity calculated from Eq. (1)) implies a $\Delta[\mathrm{Fe} / \mathrm{H}]={ }_{-0.15}^{+0.18} \mathrm{dex}$, for a star with $T=4800 \mathrm{~K}$, and $\Delta[\mathrm{Fe} / \mathrm{H}]=$ ${ }_{-0.03}^{+0.07}$ dex, for a star with $T=4300 \mathrm{~K}$. A systematic error of \pm 0.2 in the microturbulence velocity implies a $\Delta[\mathrm{Fe} / \mathrm{H}]={ }_{+0.13}^{-0.12} \mathrm{dex}$, for both cool and warm stars. A more extensive discussion of systematic errors in this kind of analysis is presented in Lecureur et al. (2008).

\subsection{Stars observed with UVES}

Figure 5 shows, for the stars observed also at high resolution with UVES, the comparison between resulting iron abundances (upper panel) and the most relevant parameters (middle and lower panels). We note that the stars observed with UVES were 58 in total (cf. Table 1 in Lecureur et al. 2007): 13 were Baade's Window clump, and another 13 were giants, 11 more giants were observed in the $b=-6^{\circ}$ field, 7 in the $b=-12^{\circ}$ field and 14 in the NGC 6553 field. However only 50 of them were discussed in Zoccali et al. (2006) and 53 in Lecureur et al. (2007) because 
those studied focused on the analysis of a few specific lines, sometimes heavily blended with telluric lines.

From the 58 UVES stars, here we exclude from Fig. 5 the 7 stars in the $b=-12^{\circ}$ field because they were never re-observed with GIRAFFE, and one more clump star that also failed to be reobserved with GIRAFFE. We are thus left with 50 data points. Among them, open symbols are stars with large dispersion in the line-by-line iron determination, mostly high metallicity stars, and most likely due to line crowding.

The systematic offset is negligible in all the panels. The scatter, again representative of the statistical error, is $\sigma[\mathrm{Fe} / \mathrm{H}]=$ \pm 0.16 , consistent with our estimates above. The largest scatter is in the adopted excitation temperature, revealing that this parameter is constrained to no better than $\pm 200 \mathrm{~K}$.

\subsection{Globular cluster stars}

Yet another independent test on the internal precision of our analysis is offered by the stars which are members of the globular clusters in these fields. The left panels of Fig. 6 show a plot of radial velocity versus metallicity for all the stars in our fields (in a narrow range of radial velocity and metallicity) together with globular cluster stars, shown here as filled triangles. The location of cluster stars in the field of view of FLAMES is shown on the right side of the plot. Cluster members were selected as target stars having $[\mathrm{Fe} / \mathrm{H}]$ within \pm 0.2 dex from the cluster mean, radial velocity within $\pm 10 \mathrm{~km} \mathrm{~s}^{-1}$ from the mean, and located within 2 arcmin from the cluster center. Baade's Window contains 7 stars belonging to the metal-poor cluster NGC 6522, and only one member of NGC 6528, at solar metallicity and radial velocity close to $200 \mathrm{~km} \mathrm{~s}^{-1}$ (not shown here). The field at $b=-6^{\circ}$ contains six members of NGC 6558 (Barbuy et al. 2007). Finally the NGC 6553 field contains the eponymous cluster, but its position in this plot falls near the center of the distribution of the field stars, thus it is harder to discriminate cluster from field, and for this reason the metallicity spread of NGC 6553 putative members is not considered here. Cluster stars should have identical velocity and composition, thus the observed spread in this plot is a measure of our (mostly statistical) error. For NGC 6522 and NGC 6558 the $1 \sigma$ spread for cluster stars is $\sigma[\mathrm{Fe} / \mathrm{H}]=0.12$ and $\sigma[\mathrm{Fe} / \mathrm{H}]=0.15$, respectively.

A complete analysis of the chemical abundances of cluster stars has been presented in Barbuy et al. (2007) for NGC 6558, and it is in preparation for NGC 6522. What we show here is the iron content of cluster stars, as measured considering them just like all the other field stars (e.g., adopted distance and reddening are the same as the mean ones for the bulge) and the details of the analysis, such as sigma clipping in Fe lines, etc., are suitable to be extended to all the target stars. For this reason, the actual metallicity of cluster stars derived here is not as accurate as it is in the dedicated papers, though well within our 1 sigma error bar. Cluster stars are shown here with the only purpose of helping estimating our error on individual $[\mathrm{Fe} / \mathrm{H}]$ measurements.

In summary, the three independent estimates of the internal error via $i$ ) repeated and independent analysis; $i$ ) comparison with the UVES results; and iii) globular cluster stars, indicate $\sigma[\mathrm{Fe} / \mathrm{H}]=0.09,0.16$, and $0.12 \mathrm{dex}$, respectively. All those estimates include the smaller $(<0.06 \mathrm{dex})$ statistical error due to line-to-line dispersion, but each of them includes only a subset of all the possible causes of errors. Putting together the different tests, and considering that some of the systematics (e.g., possible non LTE effects, errors in the model atmospheres themselves, etc.) have not been taken into account here, we can conclude that \pm 0.2 dex is a conservative estimation of our uncertainty on
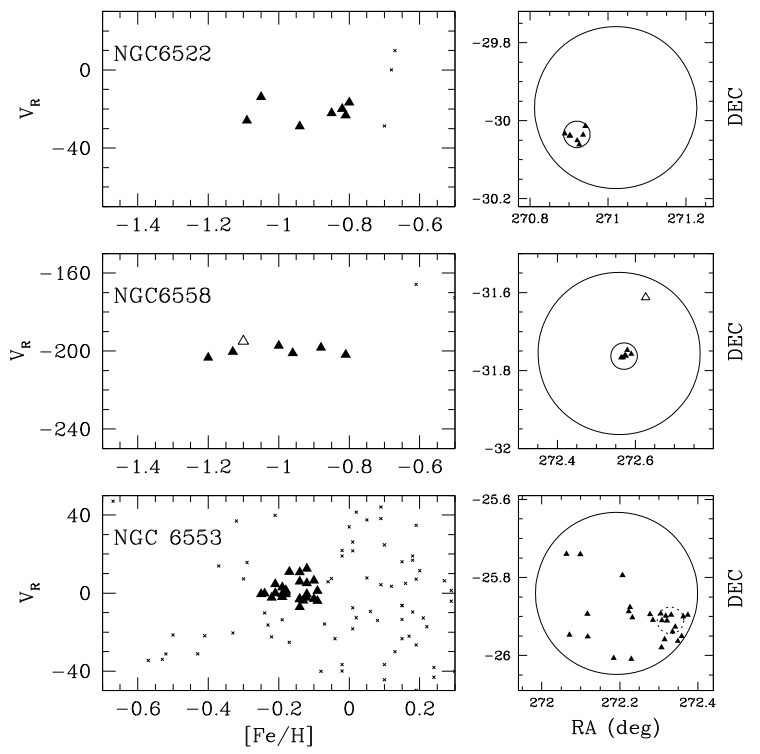

Fig. 6. Left panels: iron abundance versus radial velocity for globular cluster stars included among our targets (filled triangles). Bulge field stars are also shown as small symbols, in order to emphasize that while NGC 6522 and NGC 6558 can be easily separated from field stars, some ambiguity is present in the selection of stars belonging to NGC 6553, due to its near solar metallicity and low radial velocity. Right panels: position of cluster stars with respect to the FLAMES field of view (large circle). The small circle has a radius of 2 arcmin around the cluster center. One star, shown as an open triangle in the middle panel, has metallicity and radial velocity similar to the other NGC 6558 members, but it is very far away from the cluster center, making it unlikely to be a member. The lower right panel shows once again that unambiguous identification of cluster members in NGC 6553 is very hard.

the metallicity of the individual star, including both the effect of statistics and systematics.

\section{The distribution functions of the iron abundance}

The IDFs obtained in the three fields along the bulge minor axis are shown in Fig. 7, and the corresponding data are given in Table 3. The thick histograms show the raw IDFs, while the shaded and the thin open one are the IDF corrected from the color bias discussed in Sect. 2.1, using method i) and ii), respectively. The differences are in fact very small, fully consistent with our error bars, but we judged important to prove to ourselves that this kind of bias was negligible, given the way our targets were selected. We do not show here the IDF for the field around NGC 6553 due to the fact that, as shown in Fig. 1, this field has the strongest differential reddening, and none of the reddest stars were included in our target list. Thus, we believe that, if there is any bias, in NGC 6553 our sample may be biased against the most metal-rich stars. In addition, in order to evaluate the fraction of stars sampled at each color, we had to exclude cluster stars both in the total color magnitude diagram and in the target sample. This task proved extremely hard in the NGC 6553 field, due to the dimension and centrality of the cluster. Finally, as shown in Figs. 6 and 15, both the metallicity and the radial velocity of cluster stars sit just in the middle of the distributions of field stars. For these reasons, we will not include the IDF of this field in our discussion of the general bulge iron content. On the other hand, the NGC 6553 field, thanks to its largest extinction, will prove useful in our analysis of the disk contamination (see discussion in Sect. 8). 
Table 3. Stellar parameters and iron abundance of all the program stars.

\begin{tabular}{|c|c|c|c|c|c|c|c|c|c|c|c|c|}
\hline $\mathrm{QF}^{a}$ & ID & OGLE-ID & RA & Dec & V & $V-I$ & $\log g$ & $V_{\mathrm{t}}$ & $T_{\text {eff }}$ & {$[\mathrm{Fe} / \mathrm{H}]$} & $\sigma^{b}$ & Cluster? \\
\hline \multicolumn{13}{|c|}{ Baade's window } \\
\hline 0 & 2 & 423342 & 18:03:50.00 & $-29: 55: 45.20$ & 16.36 & 1.805 & 1.99 & 1.3 & 4650 & 0.46 & 0.38 & - \\
\hline 0 & 3 & 423323 & 18:03:48.39 & $-29: 56: 27.10$ & 16.10 & 1.846 & 1.59 & 1.5 & 4200 & -0.48 & 0.18 & - \\
\hline 0 & 4 & 412779 & 18:03:43.18 & $-29: 59: 40.10$ & 15.91 & 1.667 & 1.93 & 1.5 & 4850 & -0.37 & 0.18 & - \\
\hline 2 & 5 & 412803 & 18:03:46.14 & $-29: 58: 30.00$ & 16.40 & 2.083 & 1.52 & 1.3 & 4000 & 0.51 & 0.34 & - \\
\hline 0 & 6 & 423359 & 18:03:47.03 & $-29: 54: 49.20$ & 16.17 & 1.768 & 1.92 & 1.4 & 4650 & -1.23 & 0.23 & - \\
\hline$\ldots$ & $\ldots$ & $\ldots$ & $\ldots$ & $\ldots$ & $\ldots$ & $\ldots$ & $\ldots$ & $\ldots$ & $\ldots$ & $\ldots$ & $\ldots$ & $\ldots$ \\
\hline
\end{tabular}

${ }^{a} \mathrm{QF}$ is a subjective quality factor, classifying stars into good (0), bad (2) and intermediate (1), according to how unique/degenerate the convergence into the final model atmosphere proceeded.

${ }^{b}$ Line-to-line dispersion around the mean $[\mathrm{Fe} / \mathrm{H}]$.
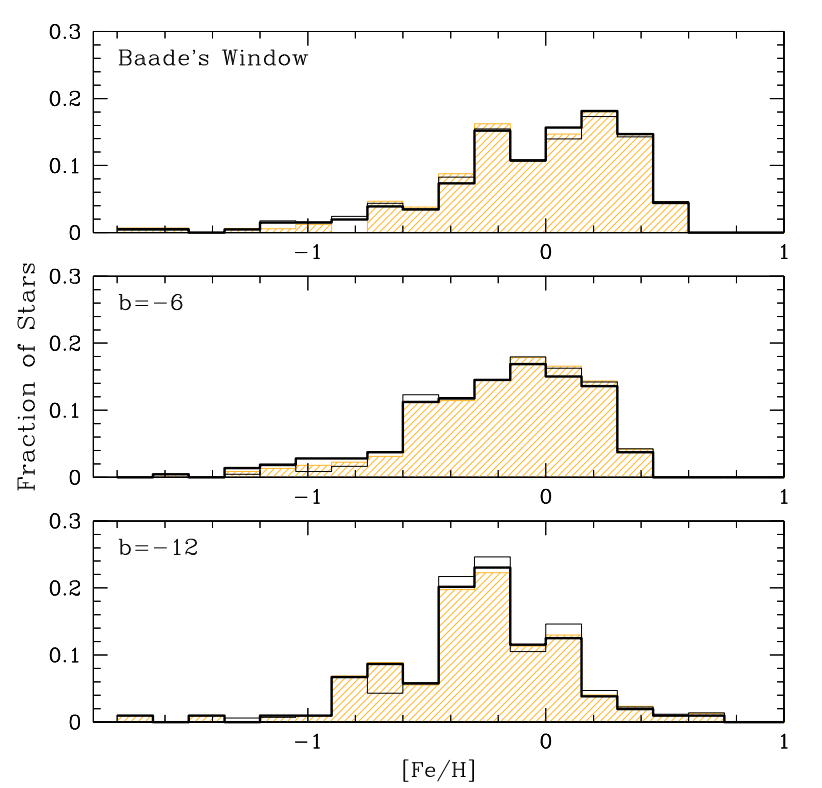

Fig. 7. The raw IDF (thick histogram) compared with the IDF corrected for color bias, according to method i) (shaded histogram) and method ii) (thin histogram) discussed in Sect. 2.1.

As mentioned before, for the Baade's Window field two independent (but homogeneous) sets of data are available: the 204 giants discussed here, and another 200 red clump giants observed within the guaranteed time reserved to the FLAMES French consortium. The latter, extensively discussed in a companion paper (Lecureur et al. 2008), have been reduced in a very similar way as the present data, and Fig. 8 shows the comparison between the IDFs of the two samples. Although some differences seem to be present between the two distributions ${ }^{4}$ Note that the smaller amount of metal poor stars in the clump IDF is expected, since metal poor stars would not be found in the red clump but on the blue side of the horizontal branch (HB). However, there are really few metal-poor stars even in the giant IDF (only 6 out of 204 stars have $[\mathrm{Fe} / \mathrm{H}]<-1.0)$ hence we consider this bias rather negligible. Therefore, in the following discussion the two sets will be combined and the quoted Baade's Window IDF will result from the independent analysis of a total of $\sim 400$ stars.

\footnotetext{
${ }^{4}$ As discussed in Lecureur et al. (2008), the analysis of the clump stars has been done with an automatic procedure, based on the same prescriptions followed here in a manual way. A discrepancy as large as that shown in Fig. 4, can be expected between the two IDFs, for the same reason, and it is still small when compared to the total uncertainty presented above: in fact, the means of the two distributions agree within 0.06 dex.
}

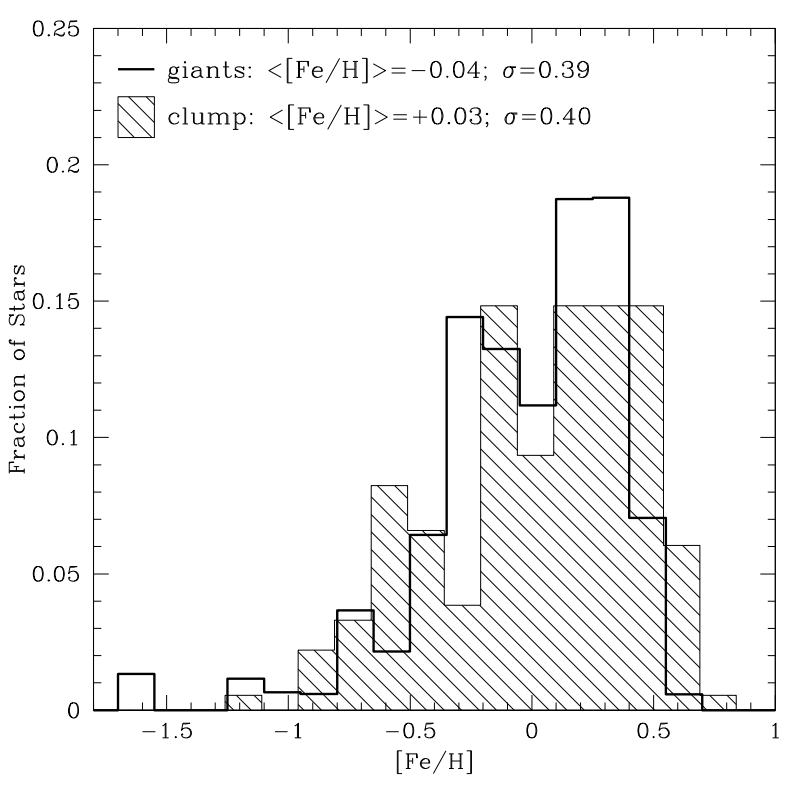

Fig. 8. Comparison between the IDF of Baade's Window as derived from giant and red clump stars, the latter from Lecureur et al. (2008).

Figure 9 shows the comparison with some of the previous determinations of the IDF of bulge fields. The left panel compares the present IDF (shaded) with the photometric one by Zoccali et al. (2003), both relative to the field at $b=-6^{\circ}$. The two IDFs are different, especially at high metallicity, possibly due to the lack of calibrating template red giant branches for solar metallicity and above. At the opposite end of the IDF, the less prominent metal poor tail with respect to Zoccali et al. (2003) can be ascribed to an innate limit of the photometric method, as the RGB color becomes less and less sensitive to $[\mathrm{Fe} / \mathrm{H}]$ at decreasing metallicity, hence even small color errors imply large errors in the derived $[\mathrm{Fe} / \mathrm{H}]$. The right panel shows the comparison with the spectroscopic IDF for Baade's Window from Fulbright et al. (2006), as obtained from the recalibration of the Sadler et al. (1996) IDF. It can be seen that in both cases the present spectroscopic IDF is appreciably narrower than previous results. In a sense, this is consistent with our effort at reducing the errors on individual measurements. However, Fig. 9 also shows as a solid histogram the 27 stars that were actually measured by Fulbright et al. (2006) at high spectroscopic resolution. Those are the stars that were used to recalibrate the Sadler et al. (1996) IDF obtained from low resolution spectra. It can be appreciated that none of the 27 stars has $[\mathrm{Fe} / \mathrm{H}]>0.5$, despite their selection of 3 stars with $[\mathrm{Fe} / \mathrm{H}] \geq 0.5$ in Sadler et al. (1996). The discrepancy at the metal rich end is in a region where the Fulbright et al. calibration 


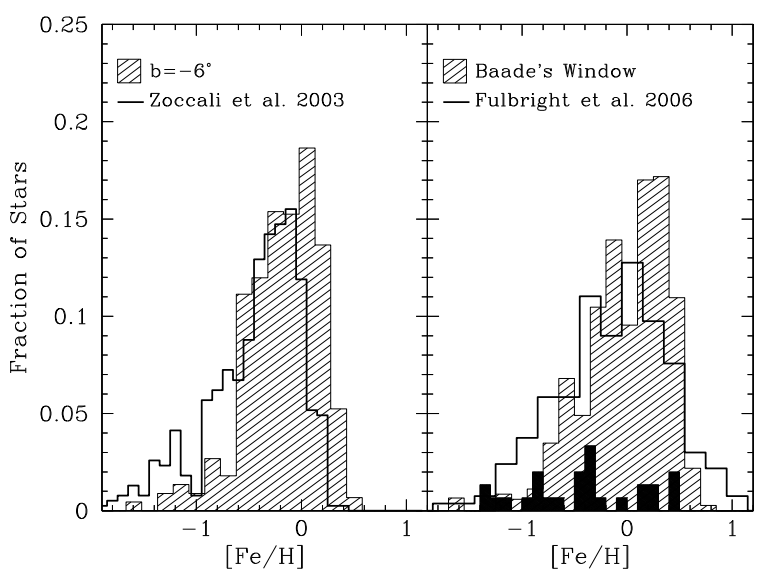

Fig. 9. The derived IDF is compared with previous measurements, in the corresponding fields. Left: the photometric IDF by Zoccali et al. (2003) obtained in the $b=-6^{\circ}$ field observed here. Right: the spectroscopic IDF by Fulbright et al. (2006) in Baade's Window is compared with the present results. Also shown as a black solid histogram (arbitrarily normalized) is the IDF of the 27 stars actually observed by Fulbright et al. (2006) at high spectral resolution.

was in fact used in extrapolation. In addition, the strong $\mathrm{Mg}_{2}$ features found in the most metal-rich and cooler stars are contaminated by TiO lines (see, e.g., Fig. 13 by Coelho et al. 2005) and the high end of the Sadler et al. (1996) IDF itself probably has an overestimated high metallicity tail.

Less obvious is the interpretation of the discrepancy at low $[\mathrm{Fe} / \mathrm{H}]$ with respect to the IDF by Fulbright et al. (2006). The high resolution sample of Fulbright et al. (2006) contains four stars with $[\mathrm{Fe} / \mathrm{H}]<-1$, so that we know that the total sample (88 giants from Rich 1988) from which those stars were picked (with an on-purpose flat IDF) had to contain at least that number of stars. This would mean that we would expect some $\sim 9$ stars in our RGB sample, whereas we observe only 6 . Although different, the two numbers are still compatible within the very low statistics considered here.

On the other hand, with some simple calculations we can check that the number of metal poor stars in the IDF is consistent with the number we expect from independent sources. First, it is well known that the bulge contains RR Lyrae stars, classical tracers of the metal poor population. From the MACHO (Alcock et al. 1998) and OGLE II (Collinge et al. 2006) surveys, we know that there are $\sim 30$ RR Lyrae per FLAMES field, at $b=-6^{\circ}$. The total number of red clump stars in this field can be estimated from the CMD in Fig. 1: there are 4090 stars within a box with $1.3<(V-I)<2.1$ and $14.5<I<15.5$. This box includes both the red clump and the RGB at that level. From the synthetic CMD presented in Zoccali et al. (2003, their Fig. 20) we know that only $67 \%$ of them, i.e., 2740 stars, are actually red clump stars. Therefore, in a FLAMES field there are 30 RR Lyrae stars for every 2740 red clump stars, i.e., $1 \%$ of the total number of stars are expected to have $[\mathrm{Fe} / \mathrm{H}]<-1$. There could still be more metal poor stars that end up too blue in the horizontal branch to pulsate as RR Lyrae. Their number can be estimated from Busso et al. (2005), who obtained spectra of candidate extreme blue HB stars in the bulge. Out of their 28 targets, 15 (57\%) turned out to be true blue HB stars. There are 51 extreme blue HB candidates in the CMD of the complete FLAMES field, hence $51 \times$ $0.57=29$ of them were confirmed spectroscopically. This number is almost identical to the number of RR Lyrae, hence another
$1 \%$ of the total number of bulge stars are expected to be metal poor enough to end up in the extreme blue HB.

All in all, based on the known fraction of stars in the extreme blue $\mathrm{HB}$ and in the RR Lyrae gap, we expect that at least $2 \%$ of the total number of stars in the bulge should be metal poor, say with $[\mathrm{Fe} / \mathrm{H}]<-1$. This percentage has to be taken as a lower limit, because while we can easily count RR Lyrae and extreme HB stars, there is a narrow range in color, corresponding to A-type blue HB stars that is heavily contaminated by the disk main sequence.

Our IDF at $b=-6^{\circ}$ is based on $\sim 200$ stars and 6 of them have $[\mathrm{Fe} / \mathrm{H}]<-1$, fully consistent with the 4 (at least) expected from the above calculation. Therefore, even if the number of metal poor stars in the IDF presented here might seem very small, for example compared with previous measurements or with a simple, closed box model (see below), it is consistent with the number of expected metal poor stars in the bulge estimated from independent evidence.

In closing this section it is worth mentioning that Johnson et al. (2007, 2008) and Cohen et al. (2008) have recently measured the chemical abundances of three bulge dwarfs during a microlensing event. They find metallicities close to $[\mathrm{Fe} / \mathrm{H}] \sim$ +0.5 for all the stars, a value too high to be consistent with random extraction of three stars from our IDF. This discrepancy is very puzzling, although it is fair to mention that several microlensed bulge dwarfs had been observed by Cavallo et al. (2003) finding metallicities consistent with ours. Speculations have been made that dwarf stars, being unevolved, might give the "true" IDF, as opposed to giants, whose evolution might actually depend on their metallicity. However, at present there is no indication that supports such major differences in the evolutionary path of a star at $[\mathrm{Fe} / \mathrm{H}]=-1.0$ with respect to one at $[\mathrm{Fe} / \mathrm{H}]=$ +0.5 . As discussed in Zoccali et al. (2003, their Fig. 13) the metallicity dependence of the evolutionary flux along the RGB (i.e., of the number of stars reaching the RGB per unit time) and of the stellar RGB lifetime has opposite trends, so that stars of all metallicities are equally represented along the RGB. Cohen et al. suggest that higher mass loss in metal rich stars would cause them to leave the RGB before reaching the level of our samples (at $I \sim 14.5$ ), then evolving to the helium white dwarf stage. Were that true, one would expect a drop in the RGB luminosity function which is not observed (Zoccali et al. 2003, their Fig. 21).

We note that the extremely high amplification of these microlensing events $(>300)$ indicates that a different amplification might have taken place between the limb and the center. The lens model and the model atmosphere should take these effects into account.

\subsection{Disk and halo contamination in the bulge fields}

In this section we present our estimates for the contamination in the survey fields coming from the thin and thick disk, and from the halo. The working tool for these estimates is an updated version of the Besançon Galaxy model (Robin et al. 2003) kindly computed by M. Schultheis for us. Simulated CMDs have been constructed for the three fields along the bulge minor axis. Small adjustments were made in the assumed reddening law in order to insure that the simulated red clump would coincide in color and magnitude with the observed one. The resulting model CMDs, together with the observed ones, are shown in the upper panels of Figs. 10-12. Clearly, the model CMDs reproduce reasonably well many characteristics of the observed CMDs, but significant differences are also evident. For example, the giant branches are 

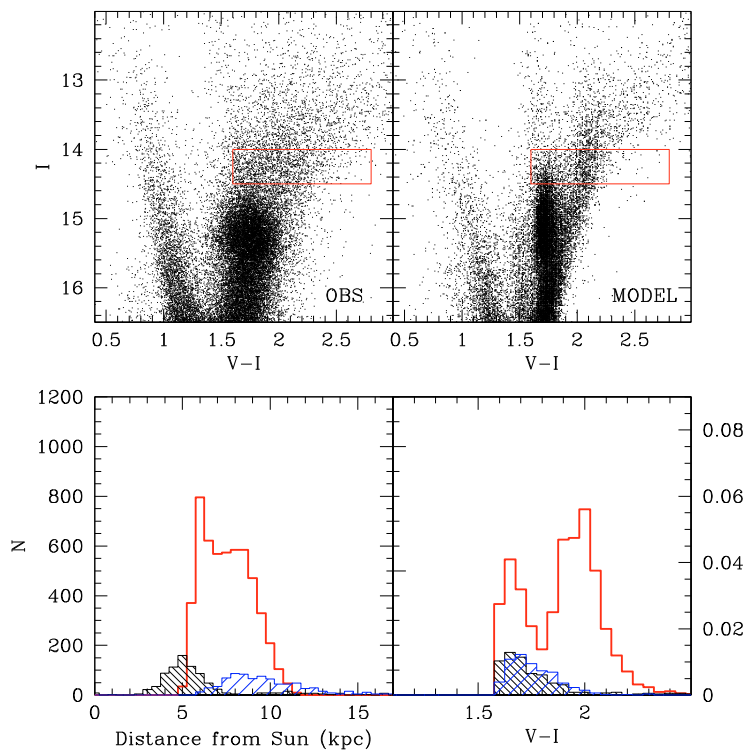

Fig. 10. The upper panels show the observed and model CMD for the Baade's Window field, together with the box where the targets were selected. The bottom panels show the distance and color distribution of bulge stars (open histogram) and of thick (light dashed) and thin disk (heavy dashed). The disk histograms are scaled to the contamination fraction - with respect to the total number of stars - shown in the $y$-axis on the right end side of the plots.
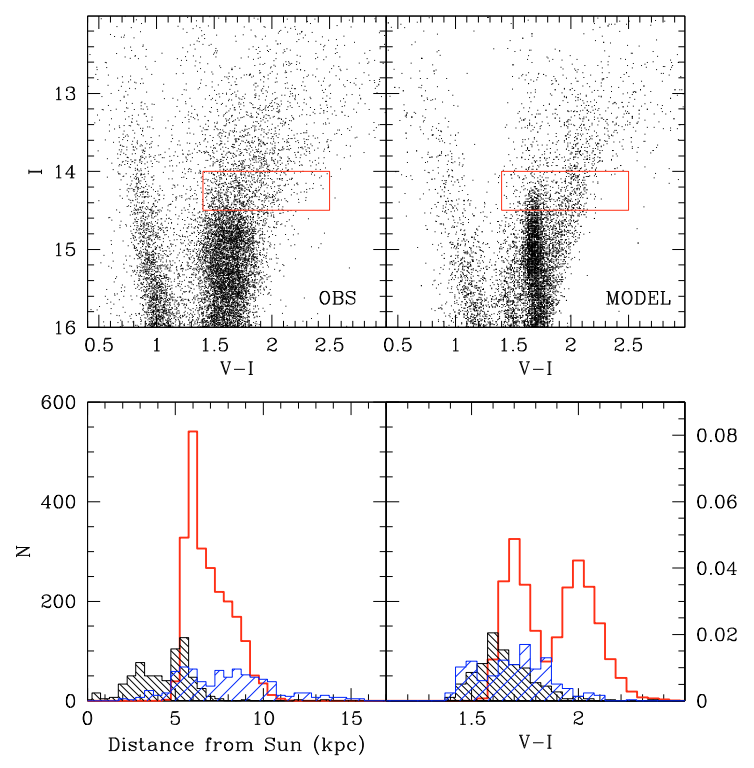

Fig. 11. Same as Fig. 10 for the field at $b=-6^{\circ}$.

much broader in the data than in the model, possibly because the model does not incorporate small scale differential reddening. Thus, the relative contributions of the various galactic components to the star samples in the various fields need to be taken with caution. However, it is still the best tool available to analyse the expected contamination of our sample from (however poorly) known galactic components on the line of sight.

Stars inside the observed target box were selected in the model CMD, and their distribution in distance, color, and stellar parameters were analysed. The lower panels of Figs. 10-12 show those distributions in distance and color. The raw histogram of bulge stars is shown here, while the disk star histograms are scaled to the fraction of the total number of stars,
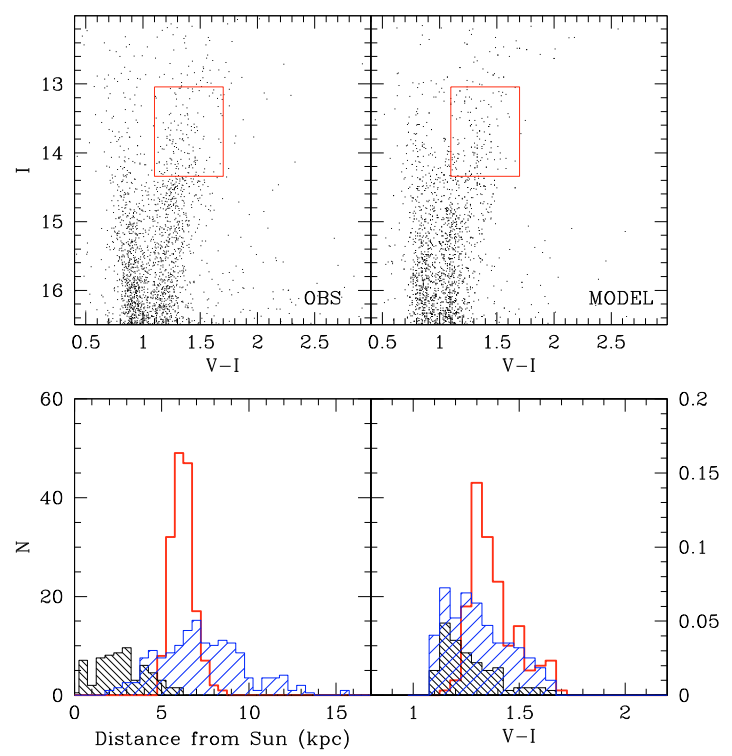

Fig. 12. Same as Fig. 10 for the field at $b=-12^{\circ}$.

reported in the scale on the right side of the lower right box. We emphasize, then, that disk and bulge here are not shown in the same scale, in order to make the disk histograms more visible. The $V-I$ histogram of bulge stars in the model shows a clear bimodality due to the inclusion of some clump stars - those on the near side of the bulge - and a small number of asymptotic giant branch stars. On the other hand the data do not show a bimodality in the color distribution. The discrepancy may be ascribed to the specific assumptions in the Besancon model, such as the red clump luminosity, color, and the bulge density law.

Particularly interesting is the distance distribution, because it helps understanding the evolutionary phase, thus the gravity, of the contaminating stars. One can see, for instance, that in Baade's Window the Besançon model predicts contaminating thick disk stars to be located at the same distance of the bulge. Therefore, for these stars the photometric gravity we assume in the analysis is correct, hence so is the derived iron abundance. On the other hand, the iron abundance alone cannot help us discriminating possible thick disk stars against the bulge ones. It is also important to remark that, if the model is correct, and the thick disk stars contaminating our sample are those as far away as the bulge (or even on the other side), our present knowledge of the thick disk characteristics (age, metallicity, scale height and density) at that position is very poor. Actually, the predicted thick disk stars within the bulge are the result of the assumption in the Besançon model that the thick disk follows an exponential radial distribution, then peaking at the Galactic center.

Contaminating foreground thin disk stars are estimated to be giant stars (not dwarfs as one might naively expect) located mostly between 2 and $5 \mathrm{kpc}$ from the Sun.

The contamination from the halo population turns out to be between $0 \%$ and $2 \%$ in all the fields (see Table 4), hence it can be safely neglected.

\section{The IDF as a function of color}

The combination of the metallicity, kinematic and color information for each target star permits a better understanding of the behaviour of the different components of the inner Galaxy.

The left panels of Fig. 13 show the IDF of the NGC 6553 field in different color bins, from blue (bottom) to red (top) as 
Table 4. Disk and halo contamination percentage in each field, relative to the total number of stars in the target box.

\begin{tabular}{lrrr}
\hline \hline Field & Thin disk & Thick disk & Halo \\
\hline Baade's Window Clump & $3.2 \pm 0.1$ & $5.8 \pm 0.1$ & $0.1 \pm 0.02$ \\
Baade's Window RGB & $6.5 \pm 0.3$ & $4.8 \pm 0.3$ & $<0.1 \pm 0.06$ \\
$b=-6^{\circ}$ Field & $9.8 \pm 0.5$ & $11.5 \pm 0.6$ & $0.4 \pm 0.10$ \\
$b=-12^{\circ}$ Field & $18.9 \pm 1.8$ & $59.0 \pm 2.1$ & $1.5 \pm 0.50$ \\
\hline
\end{tabular}
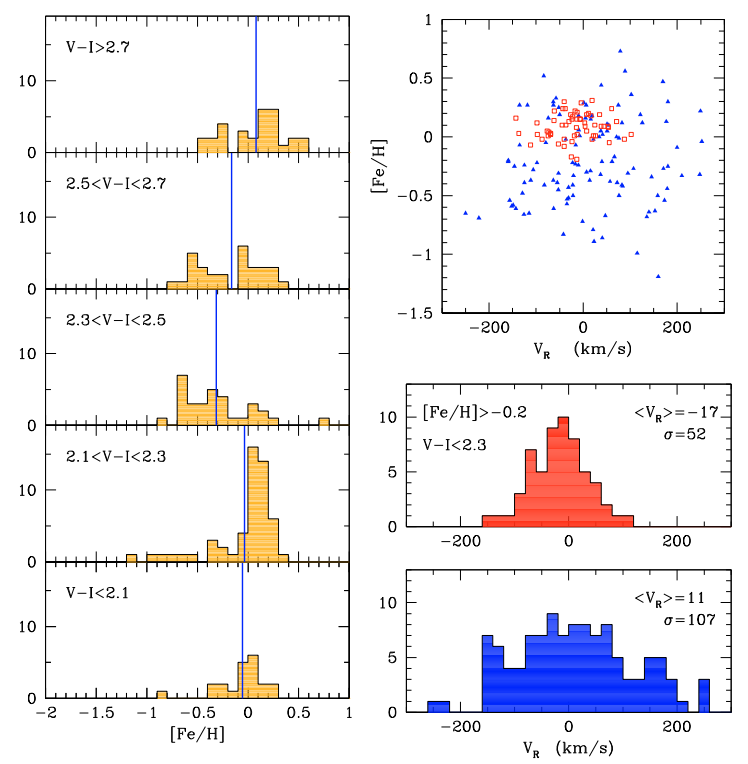

Fig. 13. Left: IDF for the NGC 6553 field as a function of color, from the bluest stars at the bottom to the reddest stars at the top. A vertical line marks the mean of the distribution. Upper right: metallicity versus radial velocity for individual stars. Cluster stars have been excluded from all these plots. Empty squares are stars with $V-I<2.3$, and $[\mathrm{Fe} / \mathrm{H}]>-0.2$, while all the other stars are filled triangles. Middle right: radial velocity distribution for stars with $V-I<2.3$ and $[\mathrm{Fe} / \mathrm{H}]>-0.2$ (empty squares above). Mean and sigma of the distribution are shown in the figure label. Bottom right, same as above for all the other field stars (small filled triangles in the upper right plot).

indicated in the labels. It is well known that, were the stars all at the same distance, i.e. belonging to the bulge, then more metal rich giants should be redder. Therefore, the IDF should be progressively shifted to the metal rich side for increasingly redder color bins (upwards in the plots), with some possible spread introduced by differential reddening. This is approximately true, except for the two bluest color bins, that unexpectedly contain only very metal rich stars. If one looks at the radial velocity distribution of those stars (open squares in the upper right plot, and middle histogram) it is clear that they are a colder distribution, with velocity dispersion of $52 \mathrm{~km} \mathrm{~s}^{-1}$. On the contrary, all the other stars, shown as filled triangles in the upper right plot, have a velocity dispersion of $107 \mathrm{~km} \mathrm{~s}^{-1}$. Note that suspected clusters stars are not included in any of these plots. Everything suggests that the bluest stars in the CMD are in fact contaminating (thin?) disk stars, located on the blue side of the target box just because they are on average closer to us. In fact, there would be no reason to expect that the most metal rich bulge stars should lie preferentially on the blue side of the CMD. Indeed, also the Besançon model predicts disk stars to be always on the blue side of our CMD target box (Figs. 10-12).

In this particular field this effect is more evident than in the other ones because of the larger interstellar extinction all along the line of sight, allowing a color separation between bulge and disk. The important conclusion that can be drawn from this exercise is that the contaminating (thin?) disk has a very metal rich IDF, quite different from that measured in the solar neighborhood. It seems that the contaminating disk is closer than the bulge (bluer in the CMD) but still quite far away from us. The existing disk radial metallicity gradient, then, may explain its higher metallicity with respect to the solar neighborhood.

\section{A radial metallicity gradient in the bulge}

The final IDFs for the three fields along the bulge minor axis are shown in Fig. 14. Overplotted to the metallicity distribution of bulge stars (histograms) are two gaussians qualitatively showing the estimated contamination by thick and thin disk. The gaussians have indeed the mean and sigma values characteristics of the thick (Reddy et al. 2006) and thin disk (Nordström et al. 2004) IDF, in the solar neighboorhood. As discussed above, very likely the contaminating disk stars are closer to the bulge than to us, but the disk radial gradient for giant stars, i.e., intermediateage and old disk, has never been measured, hence where exactly these gaussians would lie is not very well known.

Baade's Window. The IDF for this field has been derived from the combination of both clump and giant stars ( 400). Despite the uncertainty on the mean metallicity of the contaminating disk stars, it is clear that their number is negligible in this field.

Field at $b=-6^{\circ}$. The IDF for this field has been derived from 213 giant stars. Again, the relative disk contamination is low in this field, and would not have a significant impact on the shape of the derived bulge IDF. The comparison with Baade's Window reveals a difference in the mean metallicity, suggestive of a radial metallicity gradient, with the IDF mean value going from $\langle[\mathrm{Fe} / \mathrm{H}]\rangle=+0.03$ at $b=-4^{\circ}$ to $\langle[\mathrm{Fe} / \mathrm{H}]\rangle=-0.12$ at $b=-6^{\circ}$. More specifically, it would seem that rather than a solid shift towards more metal poor mean values, it is the metal rich stars that gradually disappear, while the metal poor ones are always roughly in the same position. On the same line, it is interesting to note that there is some indication of a bimodality in the IDF of this field.

Field at $b=-12^{\circ}$. The interpretation of the IDF for this field, resulting from the observations of 104 stars, is a lot more complicated due to the highest fraction of contaminating disk stars. In this case it is more important to establish what should be the mean metallicity of the contaminating stars. Regarding thick disk stars, the Besançon model predicts them to make up about $60 \%$ of the observed stars. However, if thick disk stars are as metal poor as they are seen in the solar neighboorhood, they cannot be as many, just because we do not see as many metal poor stars at all. If the inner thick disk is as metal poor as it is in the solar neighborhood, it cannot account for more than $30 \%$ of the total number of stars. This $(30 \%)$ would be the metal poor gaussian shown in the lower panel. Alternatively, thick disk stars are a bit more metal rich than in the solar neighborhood, probably because they are closer to the center (Fig. 12). Then in this case they could be as many as $60 \%$, perhaps. Regarding thin disk stars, they are expected to make the $20 \%$ of the total number of stars. In this case we are more inclined to think that they should lie at the metal rich end of the distribution, because: $i$ ) it will be shown in Fig. 13 that the contaminating thin disk seems to be indeed very metal rich; and ii) because Fig. 15 shows that there 


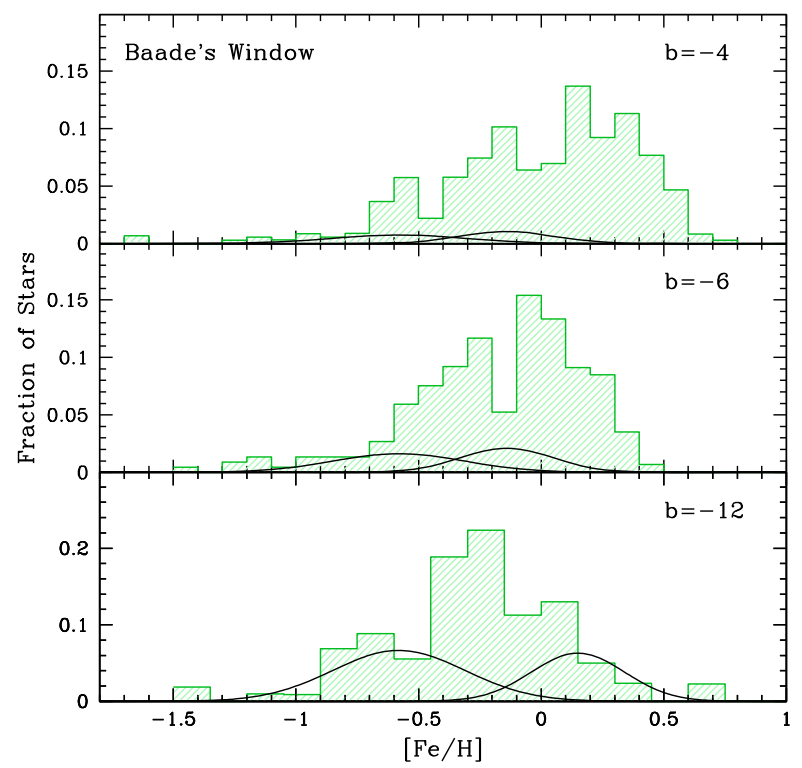

Fig. 14. The obtained IDFs for the three fields along the bulge minor axis, from the innermost one (Baade's Window, top) to the outermost one (bottom). The gaussians show the IDF of contaminating thick and thin disk stars, normalized to the expected contamination fraction, according to the Besançon Galaxy model. The thick disk contamination percent in the lower panel has been reduced at 30\% (as opposed to the $60 \%$ predicted by the model) in order to match the number of observed stars with $[\mathrm{Fe} / \mathrm{H}]<-0.5$. See text for details.

is a very cold component at the metal rich end of this field. All in all, while it is impossible to conclude what the true bulge IDF is in this field, we can conclude that the presence of the radial gradient seems confirmed in this field. Indeed, if thick disk stars are as metal poor we as we have drawn them in the figure, then the mean bulge IDF is $\langle[\mathrm{Fe} / \mathrm{H}]\rangle=-0.26$, lower than in the innermost fields. Even if thick disk stars are more metal rich, regardless of how many they are, then the mean metallicity of the remaining bulge stars can only be even lower.

The discussion above draws our attention to the fact that our knowledge of the disk properties, far away from the Sun, is still extremely poor. The Besançon model predicts a large amount of thick disk stars in the central region of our Galaxy. However, there is certainly a hole in the $\mathrm{HI}$ and $\mathrm{CO}$ distribution inside $\sim 3 \mathrm{kpc}$ (e.g., Dame et al. 2001), and we know that in most barred galaxies disk stars are cleaned up in the central region. The Besançon model does include a central hole in the thin disk distribution, but its thick disk has a pure exponential radial distribution. Does the real thick disk follow the thin disk and gas distribution, or does it keep growing toward the center?

On one hand, this emphasizes the importance of gathering more information about the inner disk, in order to understand not only the properties of the disk itself but also of other galactic components affected by disk contamination. On the other hand, the lower panel of Fig. 14, if hard to interpret in terms of bulge IDF, poses already important constraints on the properties of the inner disk. Namely, if as much as $60 \%$ of the observed stars at $b=-12^{\circ}$ belong to the thick disk, then their metallicity must be definitely higher than it is in the solar neighborhood, and possibly also much narrower.

Finally, we note that while we found indications of a radial gradient between $b=-4^{\circ}$ and $b=-12^{\circ}$, the results by Rich et al. (2007) indicate a flattening between $(l, b)=(1,-4)$ and $(l, b)=(0,-1)$. A flattening of the radial gradient in the inner
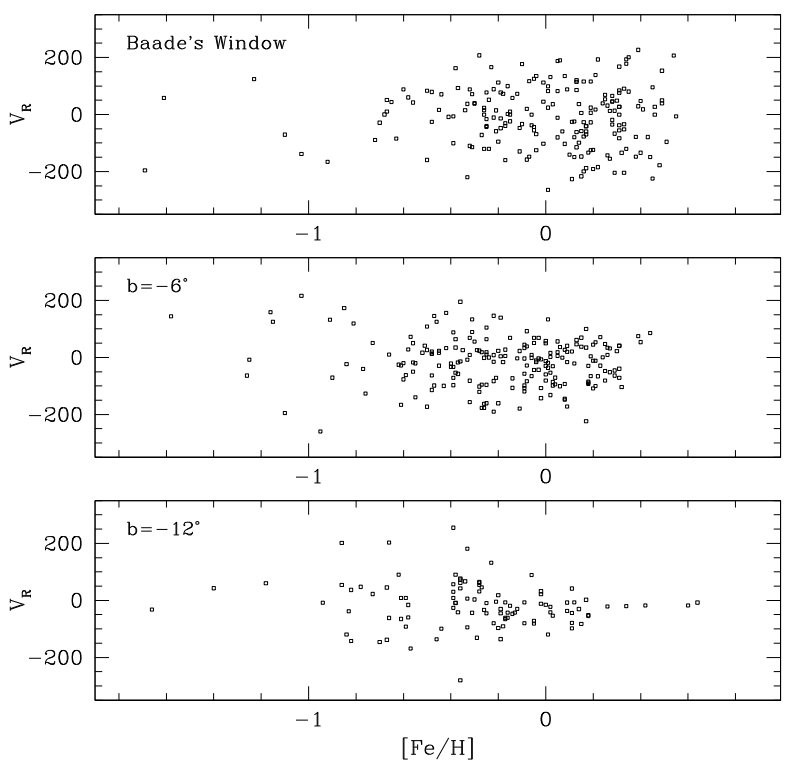

Fig. 15. Metallicity versus radial velocity for individual stars in the three bulge fields along the minor axis. Globular cluster stars shown in Fig. 6 have been removed from this plot.

bulge below $b=-4^{\circ}$ was also obtained by Ramírez et al. (2000) from low resolution spectroscopy of giant stars. Also Tyson \& Rich (1993) using Washington photometry found a radial gradient outside $b=-6^{\circ}$ and a flattening (or a slight turnover) in the inner part.

\section{Metallicity versus kinematics}

Figure 15 shows the radial velocities versus metallicity for bulge field stars in the three fields. A couple of important pieces of information can be extracted from such a plot.

First, as expected, the velocity dispersion goes down along the bulge minor axis, being $\sigma_{\mathrm{RV}}=105 \mathrm{~km} \mathrm{~s}^{-1}$ in Baade's Window, $\sigma_{\mathrm{RV}}=84 \mathrm{~km} \mathrm{~s}^{-1}$ in the $b=-6^{\circ}$ field, and $\sigma_{\mathrm{RV}}=$ $80 \mathrm{~km} \mathrm{~s}^{-1}$ in the field at $b=-12^{\circ}$. The latter would be further reduced to $\sigma_{\mathrm{RV}}=60 \mathrm{~km} \mathrm{~s}^{-1}$ if the 5 stars with absolute velocity $\left|V_{\mathrm{RV}}\right|>150 \mathrm{~km} \mathrm{~s}^{-1}$ are rejected (e.g., if they were halo stars).

Second, the velocity dispersion of the metal rich tail is extremely different in the three fields along the minor axis, being hotter than the metal-poor one in the innermost field, about the same in the intermediate one, and extremely cold in the outermost field. The latter field being heavily contaminated by disk stars, we are inclined to think that the metal rich tail is in fact made by thin disk stars (see discussion at the end of Sect. 7). On the contrary, since the two innermost fields both have negligible disk contamination, the interpretation of such a different kinematical behaviour of the metal rich component with respect to the metal poor one is not at all straightforward. A detailed analysis of the bulge kinematics from the present data will be presented in Babusiaux et al. (2008, in preparation).

\section{Discussion and conclusions}

Figure 16 shows the $[\mathrm{Fe} / \mathrm{H}]$ distribution in the Baade's Window field with superimposed the distribution function of a simple, one-zone, closed-box model of chemical evolution with an assumed iron yield $y_{\mathrm{Fe}}=+0.03$. The simple model has been normalized to 1 , plotting the fraction of the total number of stars at each metallicity, as for the observed distribution. Rich (1990) 


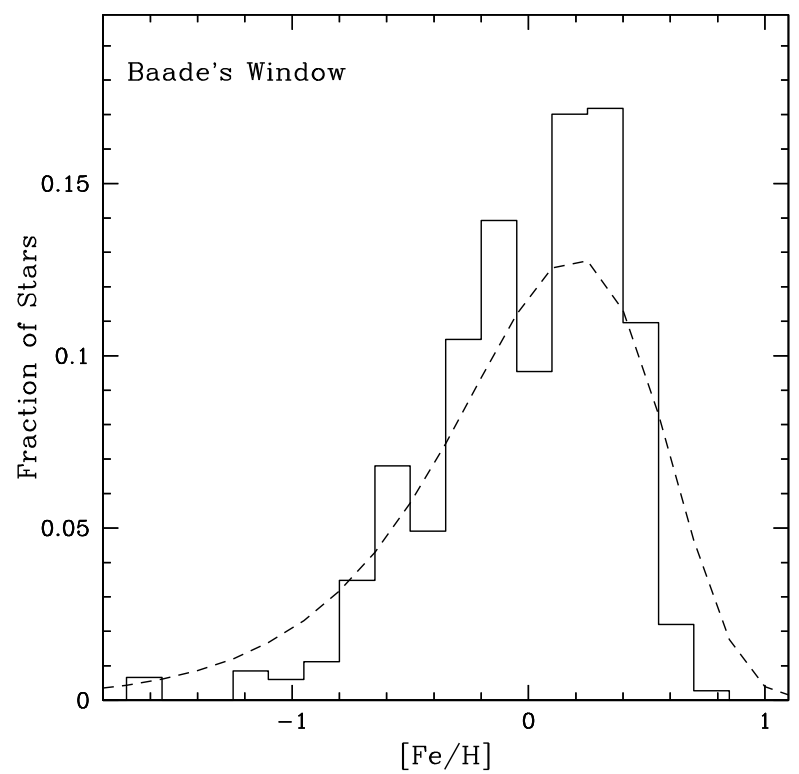

Fig. 16. The observed bulge IDF in Baade's Window compared with a simple, one-zone model with an assumed iron yield of $y_{\mathrm{Fe}}=+0.03$.

first noted that such simple chemical evolution model is a fairly good match to the bulge data, in his case the Rich (1988) data. As shown in Fig. 16, this is still the case for the data presented in this paper. However, at a closer look the match does not look perfect: the observed distribution appears to be a little narrower than the model one, and would be even narrower after deconvolving it for the observational errors. Moreover, the observed distribution shows a sharper cutoff at high metallicity, compared with the closed box model. A small deficit at the low-metallicity end with respect to the model was noticed by Zoccali et al. (2003) for their photometric IDF. A small deficit was also found by Fulbright et al. (2006) for the original Rich (1988) sample of bulge K giants, having recalibrated the old data using Keck/HIRES high resolution spectra for a subsample of the stars. But overall, they find quite good agreement with a closed-box model, once either the mean or the median iron abundance is used for the yield.

Undoubtedly, a closed-box model provides a rather satisfactory qualitative match to the data. Should we conclude that the bulge really evolved as a closed box? Certainly not. In such a model the bulge starts its evolution with its whole mass in gaseous form, and proceeds with star formation till gas exhaustion. Thus, the closed-box model describes the chemical evolution of the classic monolithic collapse model. In modern scenarios for the bulge formation, via either merging of smaller entities or via disk instabilities, the bulge is assembled gradually, rather than being already in one piece from the beginning. Thus, the bulge "box" was open with respect to gas (and stars) accretion. Moreover, most likely it was also open in the opposite direction, i.e., ejecting gas and metals via supernova/AGN driven winds. We expand here on this latter aspect.

The iron yield from theoretical stellar nucleosynthesis is subject to large uncertainties, which are difficult to reduce without help from observations. The iron yield from individual massive stars exploding as type II supernovae is critically dependent upon the precise location of the mass cut between the compact remnant and the supernova ejecta, which in fact cannot be reliably predicted. In the case of type Ia supernovae (SNIa), it is their total number (and their distribution of delay times) as a result of turning a given amount of gas into stars that can hardly be predicted only from first principles (e.g., Greggio 2005). For these reasons, an empirical estimate of the iron yield may be especially helpful. Such an opportunity can be exploited in the case of clusters of galaxies, which indeed are more likely to have retained all the stars, gas and metals that have participated in the evolution. Thus, combining the iron content of the intracluster medium from X-ray observations, with that in stars from optical observations of cluster galaxies, one finds that clusters contain $\sim 0.015 M_{\odot}$ of iron for each $B$-band solar luminosity of the cluster galaxies (Renzini 1997, 2004). We shall now explore the consequences of assuming that this empirical iron yield applies also to the Milky Way bulge.

Most of the iron in clusters of galaxies was produced by the dominant stellar population, i.e. by stars in early-type galaxies that formed at $z \gtrsim 2$ (for a review, see Renzini 2006), i.e., by galactic spheroids. With an age of over $\sim 10$ Gyr (Zoccali et al. 2003), also the stars in the bulge "formed at $z \gtrsim 2$ ", and the bulge is a spheroid. Thus, our assumption of a similar iron yield in the bulge as in clusters is quite reasonable. Now, with a present $B$-band luminosity of $\sim 6 \times 10^{9} L_{\mathrm{B}, \odot}$ (Kent et al. 1991), the bulge stellar population should have produced $\sim 6 \times 10^{9} \times 0.015=9 \times$ $10^{7} M_{\odot}$ of iron. But with a mass of $1.6 \times 10^{10} M_{\odot}($ e.g., Han $\&$ Gould 1995; Bissantz \& Gerhard 2002; Sumi et al. 2006) and a mean iron abundance (in mass) $\left\langle Z_{\mathrm{Fe}}\right\rangle=0.0018$ (as the average of the individual $Z_{\mathrm{Fe}}=Z_{\mathrm{Fe}, \odot} \times 10^{[\mathrm{Fe} / \mathrm{H}]}$ in Baade's Window) the bulge contains today only $\sim 2.9 \times 10^{7} M_{\odot}$ of iron, i.e., about a factor of 3 less than it should have produced. Therefore, under this assumption the bulge would have ejected $\sim 70 \%$ of the iron it had produced (Renzini 2004).

Chemical evolution models for the bulge that relax the closed-box approximation not only with regard to bulge assembly (as in e.g., Matteucci et al. 1999), but also allowing for bulge winds are now appearing in the literature (Ferreras et al. 2003; Ballero et al. 2007; Tsujimoto 2007). The bulge IDF predicted by Ballero et al. (2007) qualitatively agrees with the one measured here (cf., their Fig. 3). However, these models involve several free parameters, which are needed to describe the rate at which new gas (and stars) are added to the growing bulge, the star formation law, the IMF, the stellar nucleosynthesis, the distribution of delay times for SNIa's, and the onset and strengths of the winds. Some of these parameters produce similar changes on the predicted IDF making the comparison between observed and model IDF not sufficient to constrain the whole formation scenario. In addition, the models predict a global IDF for the whole bulge. Due to the presence of a radial metallicity gradient, a direct comparison with observations is not straightforward. In view of these difficulties, it is worth summarizing here what are the major, purely observational constraints on the formation and evolution of the Galactic bulge.

Zoccali et al. (2003) have shown that a simulated CMD with an age of $13 \mathrm{Gyr}$ (that includes the bulge metallicity distribution) gives a fairly good match to the bulge CMD. In particular, this good match includes the luminosity difference between the horizontal branch and the main sequence turnoff, a classical age indicator. However, due to metallicity, reddening, and distance dispersion, the bulge turnoff cannot be located to better than $0.2-0.3 \mathrm{mag}$, corresponding to an age uncertainty of 2-3 Gyr. Conservatively, we take the age of the bulk of bulge stars to be in excess of $10 \mathrm{Gyr}$, and even so this implies that star formation and chemical enrichment had to be confined within a time interval definitely shorter than the age of the universe at a lookback time of $10 \mathrm{Gyr}$, or $\$ 3.7 \mathrm{Gyr}$ according to the current concordance cosmology. If the bulk of bulge stars formed in the cosmic time interval corresponding to redshift between 3 and 2, then star formation cannot have taken much more than $\sim 1$ Gyr. 
Thus, the main uncertainty affecting the duration of the star formation in the bulge comes from the uncertainty in its age: the older the age, the shorter the star-formation era.

The second constraint on the formation timescale of the bulge comes from the observed $\alpha$-element enhancement (McWilliam \& Rich 1994, 2003; Barbuy et al. 2006; Zoccali et al. 2004, 2006; Lecureur et al. 2007; Fulbright et al. 2007), once this is interpreted as a result of the interplay of the fast delivery of iron-poor nucleosynthesis products of massive stars by SNII's, with the slow delivery of iron-rich products by SNIa's. Again, a star formation timescale of approximately $1 \mathrm{Gyr}$ is generally derived from chemical evolution models, which typically assume a distribution of SNIa delay times from Greggio \& Renzini (1983). Thus, the derived timescale is modulo the adopted distribution of SNIa delay times. Other equally plausible distributions (e.g. Greggio 2005) would have given shorter or longer timescales. Thus, until the actual mix of SNIa progenitors is fully identified, we shall remain with this uncertainty on how to translate an $\alpha$-element overabundance into a star formation timescale. All in all, combining the age and the $\alpha$-element enhancement constraints, it is fair to conclude that the formation of the bulge cannot have taken much more that $~ 1 \mathrm{Gyr}$, and possibly somewhat less than that.

In addition, the indications of a radial metallicity gradient found here would argue against the formation via secular evolution of the bar, because obviously the vertical heating that transforms a bar into a pseudo-bulge would not act preferentially on metal poor stars. However, combining our result with previous ones on the inner bulge, at the moment there is evidence of a flat metallicity distribution inside $\sim 600 \mathrm{pc}$, and a radial gradient outside. Should those findings be confirmed, they might indicate the presence of a double-component bulge, an inner pseudo-bulge, and an outer classical one, as already found by Peletier et al. (2007) within the SAURON survey of galaxy bulges.

Finally, concerning the bulge chemical evolution, from the IDF we can certainly conclude that the bulge must have accreted primordial gas, due to the lack of metal poor stars with respect to the simple model prediction (the so-called $\mathrm{G}$ dwarf problem, solved with the inclusion of some infall in the model) and must have ejected a substantial fraction of the iron it produced (outflow). In addition, from the overabundance of $\alpha$-elements quoted above we can conclude that it cannot have accreted stars already significantly enriched by SNIa products, such as disk stars, or stars born in small galactic entities similar to the surviving satellite galaxies in the Local Group (e.g., Venn et al. 2004).

Acknowledgements. We thank Yazan Momany for providing the astrometric and photometric catalogue for the NGC 6553 field. We thank Mathias Schultheis for providing us the results of an updated version of the Besançon Galaxy model. This work has been partly funded by the FONDAP Center for Astrophysics 15010003 (M.Z. and D.M.) and by Projecto FONDECYT Regular \#1085278. D.M. and B.B. acknowledge the European Commission's ALFA-II programme, through its funding of the Latin-american European Network for Astrophysics and Cosmology (LENAC). B.B. acknowledges grants from CNPq and Fapesp. S.O. acknowledges the Italian Ministero dell'Università e della Ricerca Scientifica e Tecnologica (MURST) under the program 'Fasi iniziali di evoluzione dell'alone e del bulge Galattico' (Italy).

\section{References}

Alcock, C., Allsman, R. A., Alves, D. R., et al. 1998, ApJ, 492, 190 Alonso, A., Arribas, S., \& Martínez-Roger, C. 1999, A\&AS, 140, 261 Alvarez, R., \& Plez, B. 1998, A\&A, 330, 1109

Bagnulo, S., Jehin, E., Ledoux, C., et al. 2003, Messenger, 114, 10 Ballero, S. K., Matteucci, F., Origlia, L., \& Rich, R. M. 2007, A\&A, 467, 123 Barbuy, B., Perrin, M.-N., Katz, D., et al. 2003, A\&A, 404, 661

Barbuy, B., Zoccali, M., Hill, V., et al. 2006, ESO Astrophys. Symp., ed. S. Randich, \& L. Pasquini, 87
Barbuy, B., Zoccali, M., Ortolani, S., et al. 2007, AJ, 134, 1613

Barklem, P. S., Anstee, S. D., \& O’Mara, B. J. 1998, PASA, 15, 336

Barklem, P. S., Piskunov, N. E., \& O’Mara, B. J. 2000, A\&AS, 142, 467

Blecha, A., North, P., Royer, F., \& Simond, G. 2003, BLDR Software Reference Manual 1.09, VLT-SPE-OGL-13730-0040, http://www.eso. org/instruments/flames/manuals.html

Bissantz, N., \& Gerhard, O. 2002, MNRAS, 330, 591

Busso, G., Moehler, S., Zoccali, M., Heber, U., \& Yi, S. K. 2005, ApJ, 633, L29 Carpenter, J. M. 2001, AJ, 121, 2851

Cavallo, R. M., Cook, K. H., Minniti, D., \& Vanderhei, T. 2003, in Discoveries and Research Prospects from 6- to 10-Meter-Class Telescopes II, ed. P. Guhathakurta, Proc. SPIE, 4834, 66 [arXiv: astro-ph/0209196]

Coelho, P., Barbuy, B., Meléndez, J., Schiavon, R. P., \& Castilho, B. V. 2005, A\&A, 443, 735

Cohen, J. G., Huang, W., Udalski, A., Gould, A., \& Johnson, J. 2008, AJ, submitted [arXiv: 0801.3264]

Collinge, M. J., Sumi, T., \& Fabrycky, D. 2006, ApJ, 651, 197

Dame, T. M., Hartman, D., \& Thaddeus, P. 2001, ApJ, 547, 792

Feltzing, F., \& Gilmore, G. 2000, A\&A, 355, 949

Ferreras, I., Wyse, R. F. G., \& Silk, J. 2003, MNRAS, 345, 1381

Fuhr, J. R., \& Wiese, W. L. 2006, JPCRD, 35, 1669

Fulbright, J. P., McWilliam, A., \& Rich, R. M. 2006, ApJ, 636, 821

Fulbright, J. P., McWilliam, A., \& Rich, R. M. 2007, ApJ, 661, 1152

Gratton, R. G., \& Sneden, C. 1990, A\&A, 234, 366

Greggio, L. 2005, A\&A, 441, 1055

Greggio, L., \& Renzini, A. 1983, A\&A, 118, 217

Gustafsson, B., Edvardsson, B., Eriksson, K., et al. 2002, in Stellar Atmosphere Modeling, ed. I. Hubeny, D. Mihalas, \& K. Werner, ASP Conf. Ser., 288, 331 Han, C., \& Gould, A. 1995, ApJ, 449, 521

Ibata, R. A., \& Gilmore, G. 1995a, MNRAS, 275, 591

Ibata, R. A., \& Gilmore, G. 1995b, MNRAS, 275, 605

Johnson, A. J., Gal-Yam, A., Leonard, D. C., et al. 2007, ApJ, 655, L33

Johnson, A. J., Gaudi, B. S., Sumi, T., Bond, I. A., \& Gould, A. 2008, AJ, submitted [arXiv:0801.2159]

Kent, S. M., Dame, T. M., \& Fazio, G. 1991, ApJ, 378, 131

Kormendy, J., \& Kennicutt, R. C. Jr. 2004, ARA\&A, 42, 603

Kuijken, K., \& Rich, R. M. 2002, AJ, 123, 2054

Lecureur, A., Hill, V., Zoccali, M., et al. 2007, A\&A, 465, 799

Lecureur, A., Hill, V., Zoccali, M., et al. 2008, A\&A, in preparation

Magain, P. 1984, A\&A, 134, 189

Martin, W. C., et al. 2002, NIST Atomic Database, version 2.0 (Gaithersburg,

MD: National Institute of Standards and Technology), http://physics . nist.gov/asd

Matteucci, F., Romano, D., \& Molaro, P. 1999, A\&A, 341, 458

Minniti, D. 1996, ApJ, 459, 175

Momany, Y., Vandame, B., Zaggia, S., et al. 2001, A\&A, 379, 436

McWilliam, A., \& Rich, R. M. 1994, ApJ, 91, 749

McWilliam, A., \& Rich, R. M. 2003, in Origin and Evolution of the Elements (http://www.ociw.edu/ociw/symposia/series/symposium4/ proceedings.html)

Nordström, B., Mayor, M., Andersen, J., et al. 2004, A\&A, 418, 989

Ortolani, S., Renzini, A., Gilmozzi, R., et al. 1995, Nature, 377, 701

Pasquini, L., Alonso, J., Avila, G., et al. 2003, SPIE, 484, 1682

Peletier, R. F., Falco'n-Barroso, J., Bacon, R., et al. 2007, MNRAS, 379, 445

Ramírez, I., \& Meléndez, J. 2005, ApJ, 626, 465

Ramírez, S. V., Stephens, A. W., Frogel, J. A., \& DePoy, D. L. 2000, AJ, 120, 833

Reddy, B. E., Lambert, D. L., \& Allende Prieto, C. 2006, MNRAS, 367, 1329

Renzini, A. 1997, ApJ, 488, 35

Renzini, A. 2004, in Clusters of Galaxies, ed. J. S. Mulchaey, A. Dressler, \& A. Oemler (Cambridge, CUO), 16

Renzini, A. 2006, ARA\&A, 44, 141

Rich, R. M. 1988, AJ, 95, 828

Rich, R. M. 1990, ApJ, 362, 604

Rich, R. M., \& Origlia, L. 2005, ApJ, 634, 1293

Rich, R. M., Origlia, L., \& Valenti, E. 2007, ApJ, 665, L119

Robin, A. C., Reylé, C., Derrière, S., \& Picaud, S. 2003, A\&A, 409, 523

Sadler, E. M., Rich, R. M., \& Terndrup, D. M. 1996, AJ, 112, 171

Spite, M. 1967, Annales d'Astroph., 30, 211

Sumi, T., Woźniak, P. R., Udalski, A., et al. 2006, ApJ, 636, 240

Tsujimoto, T. 2007, ApJ, 665, L115

Tyson, N. D., \& Rich, R. M. 1993, in Galactic Bulges, ed. DeJonghe, \& Habing (Dordrecht: Kluwer), IAU Symp., 153, 333

Udalski, A., Szymanski, M., Kubiak, M., et al. 2002, Acta Astron., 52, 217

Venn, K. A., Irwin, M., Shetrone, M. D., et al. 2004, AJ, 128, 1177

Zoccali, M., Renzini, A., Ortolani, S., et al. 2003, A\&A, 399, 931

Zoccali, M., Barbuy, B., Hill, V., et al. 2004, A\&A, 423, 507

Zoccali, M., Lecureur, A., Barbuy, B., et al. 2006, A\&A, 457, L1 
Table 3. Stellar parameters and iron abundance of all the program stars.

\begin{tabular}{|c|c|c|c|c|c|c|c|c|c|c|c|c|}
\hline $\mathrm{QF}^{a}$ & ID & OGLE-ID & RA & Dec & V & $V-I$ & $\log g$ & $V_{\mathrm{t}}$ & $T_{\text {eff }}$ & {$[\mathrm{Fe} / \mathrm{H}]$} & $\sigma^{b}$ & Cluster? \\
\hline \multicolumn{13}{|c|}{ Baade's window } \\
\hline 0 & 2 & 423342 & 18:03:50.00 & $-29: 55: 45.20$ & 16.36 & 1.805 & 1.99 & 1.3 & 4650 & 0.46 & 0.38 & - \\
\hline 0 & 3 & 423323 & 18:03:48.39 & $-29: 56: 27.10$ & 16.10 & 1.846 & 1.59 & 1.5 & 4200 & -0.48 & 0.18 & - \\
\hline 0 & 4 & 412779 & 18:03:43.18 & $-29: 59: 40.10$ & 15.91 & 1.667 & 1.93 & 1.5 & 4850 & -0.37 & 0.18 & - \\
\hline 2 & 5 & 412803 & 18:03:46.14 & $-29: 58: 30.00$ & 16.40 & 2.083 & 1.52 & 1.3 & 4000 & 0.51 & 0.34 & - \\
\hline 0 & 6 & 423359 & 18:03:47.03 & $-29: 54: 49.20$ & 16.17 & 1.768 & 1.92 & 1.4 & 4650 & -1.23 & 0.23 & - \\
\hline 2 & 7 & 433669 & 18:03:37.14 & $-29: 54: 22.30$ & 16.14 & 1.854 & 1.67 & 1.5 & 4300 & 0.54 & 0.37 & - \\
\hline 0 & 8 & 412752 & 18:03:46.04 & $-30: 00: 50.90$ & 15.99 & 1.606 & 1.98 & 1.5 & 4900 & -0.80 & 0.15 & NGC 6522 \\
\hline 0 & 9 & 412794 & 18:03:44.84 & $-29: 58: 51.40$ & 16.31 & 1.823 & 1.94 & 1.3 & 4600 & 0.13 & 0.25 & - \\
\hline 0 & 11 & 402327 & 18:03:49.92 & $-30: 03: 35.30$ & 16.15 & 1.691 & 2.00 & 1.2 & 4800 & 0.15 & 0.21 & - \\
\hline 1 & 14 & 412924 & 18:03:47.33 & $-29: 59: 48.30$ & 16.29 & 1.660 & 2.05 & 1.5 & 4800 & 0.48 & 0.34 & - \\
\hline 0 & 15 & 575317 & 18:04:01.48 & $-29: 56: 23.60$ & 15.98 & 1.677 & 1.78 & 1.4 & 4550 & 0.22 & 0.26 & - \\
\hline 1 & 16 & 92600 & 18:04:07.46 & $-29: 54: 59.70$ & 16.29 & 2.050 & 1.70 & 1.0 & 4250 & 0.05 & 0.30 & - \\
\hline 0 & 17 & 412759 & 18:03:47.35 & $-30: 00: 36.40$ & 15.97 & 1.649 & 1.98 & 1.4 & 4900 & -0.39 & 0.11 & - \\
\hline 2 & 21 & 575356 & 18:03:54.82 & $-29: 55: 10.40$ & 16.38 & 2.065 & 1.56 & 1.4 & 4050 & 0.39 & 0.35 & - \\
\hline 0 & 22 & 423331 & 18:03:51.67 & $-29: 56: 17.60$ & 16.31 & 1.854 & 1.88 & 1.5 & 4500 & 0.18 & 0.25 & - \\
\hline 1 & 24 & 564797 & 18:04:03.20 & $-29: 58: 59.60$ & 16.37 & 2.025 & 1.69 & 1.5 & 4200 & 0.24 & 0.29 & - \\
\hline 0 & 25 & 564792 & 18:04:06.45 & $-29: 59: 13.70$ & 16.11 & 1.631 & 2.09 & 1.4 & 5000 & -0.68 & 0.21 & - \\
\hline 0 & 26 & 412931 & 18:03:51.32 & $-29: 59: 43.10$ & 16.36 & 1.867 & 1.87 & 1.3 & 4450 & -0.15 & 0.20 & - \\
\hline 0 & 27 & 564988 & 18:03:53.94 & $-29: 59: 29.90$ & 16.33 & 1.751 & 2.04 & 1.4 & 4750 & -0.24 & 0.18 & - \\
\hline 0 & 30 & 412792 & 18:03:47.65 & $-29: 58: 55.10$ & 16.27 & 1.859 & 1.83 & 1.4 & 4450 & -0.26 & 0.18 & - \\
\hline 0 & 31 & 564762 & 18:03:53.30 & $-30: 00: 26.50$ & 15.98 & 1.728 & 1.87 & 1.6 & 4700 & -0.63 & 0.12 & - \\
\hline 0 & 33 & 564757 & 18:04:05.96 & $-30: 00: 43.90$ & 16.18 & 1.627 & 2.01 & 1.3 & 4800 & 0.38 & 0.22 & - \\
\hline 0 & 35 & 564807 & 18:04:00.26 & $-29: 58: 24.80$ & 16.09 & 1.690 & 2.00 & 1.5 & 4850 & -0.67 & 0.15 & - \\
\hline 0 & 37 & 575293 & 18:04:04.43 & $-29: 57: 16.30$ & 16.34 & 1.842 & 1.79 & 1.3 & 4450 & 0.41 & 0.43 & - \\
\hline 0 & 38 & 92537 & 18:04:18.99 & $-29: 57: 52.60$ & 16.13 & 1.864 & 1.81 & 1.3 & 4500 & -0.56 & 0.13 & - \\
\hline 0 & 39 & 575303 & 18:04:04.81 & $-29: 56: 55.20$ & 16.14 & 1.580 & 2.02 & 1.5 & 4850 & -0.27 & 0.14 & - \\
\hline 0 & 40 & 240260 & 18:04:39.62 & $-29: 55: 19.80$ & 15.88 & 1.601 & 2.07 & 1.4 & 5150 & -0.26 & 0.14 & - \\
\hline 0 & 41 & 82762 & 18:04:20.52 & $-29: 58: 13.10$ & 16.21 & 1.769 & 1.81 & 1.4 & 4450 & 0.31 & 0.28 & - \\
\hline 0 & 42 & 92565 & 18:04:17.20 & $-29: 56: 49.10$ & 16.38 & 2.081 & 1.84 & 1.5 & 4400 & -0.05 & 0.22 & - \\
\hline 0 & 43 & 240210 & 18:04:28.97 & $-29: 57: 36.70$ & 16.16 & 1.782 & 2.00 & 1.2 & 4800 & -0.04 & 0.22 & - \\
\hline 0 & 44 & 554722 & 18:04:03.71 & $-30: 01: 33.00$ & 16.12 & 1.811 & 1.67 & 1.6 & 4600 & -0.44 & 0.14 & - \\
\hline 0 & 45 & 82725 & 18:04:10.12 & $-29: 59: 45.80$ & 16.19 & 1.673 & 1.98 & 1.3 & 4750 & -0.70 & 0.16 & - \\
\hline 0 & 46 & 231262 & 18:04:33.97 & $-29: 59: 54.30$ & 16.29 & 1.636 & 2.04 & 1.4 & 4930 & -0.10 & 0.18 & - \\
\hline 0 & 47 & 231099 & 18:04:27.24 & $-30: 01: 10.30$ & 15.90 & 1.527 & 2.06 & 1.6 & 5100 & -0.22 & 0.13 & - \\
\hline 0 & 48 & 82747 & 18:04:09.88 & $-29: 58: 51.80$ & 16.03 & 1.656 & 2.06 & 1.3 & 5000 & -0.26 & 0.16 & - \\
\hline 0 & 49 & 63856 & 18:04:11.16 & $-30: 05: 18.70$ & 16.33 & 1.814 & 2.01 & 1.3 & 4700 & 0.33 & 0.26 & - \\
\hline 0 & 50 & 231144 & $18: 04: 27.23$ & $-29: 58: 56.50$ & 16.15 & 1.793 & 1.94 & 1.5 & 4700 & -0.20 & 0.18 & - \\
\hline 0 & 53 & 231364 & 18:04:34.47 & $-29: 58: 24.70$ & 16.14 & 1.693 & 1.99 & 1.5 & 4800 & 0.27 & 0.25 & - \\
\hline 1 & 54 & 82742 & 18:04:13.80 & $-29: 59: 13.40$ & 15.98 & 1.746 & 1.68 & 1.5 & 4400 & 0.17 & 0.31 & - \\
\hline 1 & 55 & 73506 & 18:04:08.81 & $-30: 02: 03.30$ & 16.32 & 2.036 & 1.67 & 1.5 & 4200 & -0.24 & 0.25 & - \\
\hline 0 & 56 & 222451 & 18:04:23.77 & $-30: 02: 23.50$ & 16.08 & 1.759 & 1.94 & 1.3 & 4750 & -0.33 & 0.20 & - \\
\hline 0 & 57 & 73504 & 18:04:08.02 & $-30: 02: 16.40$ & 16.33 & 1.957 & 1.92 & 1.4 & 4550 & -0.16 & 0.19 & - \\
\hline 0 & 58 & 82761 & 18:04:16.23 & $-29: 58: 16.30$ & 16.18 & 1.755 & 2.01 & 1.5 & 4800 & -0.21 & 0.15 & - \\
\hline 1 & 59 & 73490 & 18:04:11.50 & $-30: 02: 56.10$ & 16.31 & 1.984 & 1.74 & 1.2 & 4300 & 0.49 & 0.37 & - \\
\hline 0 & 60 & 222618 & 18:04:33.22 & $-30: 02: 11.70$ & 16.23 & 1.825 & 2.03 & 1.4 & 4800 & -0.33 & 0.19 & - \\
\hline 2 & 61 & 357480 & 18:04:43.92 & $-30: 03: 15.20$ & 16.31 & 1.796 & 2.06 & 1.4 & 4800 & 0.32 & 0.37 & - \\
\hline 0 & 62 & 554664 & 18:04:05.38 & $-30: 04: 09.30$ & 16.22 & 1.927 & 1.91 & 1.5 & 4600 & -0.48 & 0.17 & - \\
\hline 0 & 64 & 73514 & 18:04:09.99 & $-30: 01: 42.40$ & 16.13 & 1.705 & 2.04 & 1.5 & 4900 & -0.41 & 0.18 & - \\
\hline 2 & 65 & 205243 & 18:04:35.02 & $-30: 10: 55.30$ & 16.34 & 1.903 & 2.13 & 1.4 & 4900 & 0.31 & 0.41 & - \\
\hline 0 & 66 & 82705 & 18:04:14.25 & $-30: 01: 11.20$ & 16.10 & 1.883 & 1.80 & 1.4 & 4500 & -0.19 & 0.19 & - \\
\hline 1 & 68 & 205257 & 18:04:31.86 & $-30: 10: 09.90$ & 16.23 & 1.813 & 1.94 & 1.5 & 4600 & -1.10 & 0.26 & - \\
\hline 0 & 69 & 82831 & 18:04:18.85 & $-30: 00: 35.80$ & 16.19 & 1.673 & 1.99 & 1.4 & 4750 & 0.33 & 0.27 & - \\
\hline 0 & 71 & 205436 & 18:04:28.84 & $-30: 08: 58.10$ & 16.30 & 1.662 & 2.27 & 1.4 & 5200 & 0.16 & 0.20 & - \\
\hline 0 & 72 & 82798 & 18:04:10.78 & $-30: 01: 10.00$ & 16.25 & 1.593 & 2.17 & 1.1 & 5050 & -0.06 & 0.17 & - \\
\hline 0 & 73 & 73515 & 18:04:15.53 & $-30: 01: 42.60$ & 16.05 & 1.814 & 1.81 & 1.4 & 4550 & -0.45 & 0.15 & - \\
\hline 1 & 74 & 214035 & 18:04:23.37 & $-30: 06: 30.60$ & 16.17 & 1.803 & 1.92 & 1.4 & 4650 & 0.26 & 0.42 & - \\
\hline 0 & 76 & 63794 & 18:04:14.65 & $-30: 08: 38.50$ & 16.22 & 1.868 & 2.00 & 1.3 & 4750 & -0.31 & 0.22 & - \\
\hline 0 & 77 & 63792 & 18:04:19.86 & $-30: 08: 40.80$ & 16.23 & 1.917 & 1.82 & 1.3 & 4450 & -0.15 & 0.22 & - \\
\hline 0 & 78 & 54167 & 18:04:14.82 & $-30: 11: 45.40$ & 16.30 & 1.685 & 2.06 & 1.4 & 4800 & -0.38 & 0.23 & - \\
\hline 2 & 79 & 54104 & 18:04:20.20 & $-30: 09: 59.00$ & 16.39 & 1.976 & 1.95 & 1.5 & 4550 & -0.28 & 0.36 & - \\
\hline
\end{tabular}


Table 3. continued.

\begin{tabular}{|c|c|c|c|c|c|c|c|c|c|c|c|c|}
\hline $\mathrm{QF}^{a}$ & ID & OGLE-ID & RA & Dec & $V$ & $V-I$ & $\log g$ & $V_{\mathrm{t}}$ & $T_{\text {eff }}$ & {$[\mathrm{Fe} / \mathrm{H}]$} & $\sigma^{b}$ & Cluster? \\
\hline 0 & 80 & 54132 & $18: 04: 21.69$ & $-30: 08: 55.90$ & 16.11 & 1.677 & 2.06 & 1.4 & 4950 & -0.11 & 0.23 & - \\
\hline 1 & 81 & 54273 & 18:04:14.72 & $-30: 10: 15.80$ & 16.39 & 1.700 & 2.12 & 1.3 & 4850 & 0.45 & 0.34 & - \\
\hline 0 & 82 & 44560 & 18:04:23.04 & $-30: 12: 45.30$ & 16.35 & 1.904 & 1.93 & 1.4 & 4550 & -0.23 & 0.23 & - \\
\hline 0 & 83 & 205356 & 18:04:24.17 & $-30: 10: 35.30$ & 16.36 & 1.700 & 2.16 & 1.5 & 4950 & -0.19 & 0.24 & - \\
\hline 0 & 85 & 63800 & 18:04:09.87 & $-30: 08: 21.70$ & 15.99 & 1.667 & 1.96 & 1.5 & 4850 & 0.31 & 0.25 & - \\
\hline 0 & 86 & 63849 & 18:04:07.60 & $-30: 05: 42.10$ & 16.16 & 1.784 & 1.97 & 1.4 & 4750 & -0.92 & 0.19 & - \\
\hline 1 & 87 & 537070 & 18:04:01.40 & $-30: 10: 20.70$ & 16.07 & 1.693 & 2.14 & 1.1 & 5150 & -1.03 & 0.16 & - \\
\hline 0 & 88 & 63823 & 18:04:14.08 & $-30: 07: 31.10$ & 16.19 & 1.925 & 1.87 & 1.4 & 4550 & -0.04 & 0.20 & - \\
\hline 0 & 90 & 545401 & 18:03:54.55 & $-30: 06: 35.50$ & 16.23 & 1.629 & 2.22 & 1.4 & 5150 & 0.01 & 0.19 & - \\
\hline 0 & 91 & 545440 & 18:03:59.36 & $-30: 06: 02.20$ & 16.38 & 1.931 & 1.91 & 1.5 & 4500 & -0.60 & 0.18 & - \\
\hline 0 & 92 & 54311 & 18:04:12.68 & $-30: 09: 40.70$ & 16.39 & 1.697 & 2.15 & 1.5 & 4900 & 0.26 & 0.24 & - \\
\hline 0 & 93 & 537101 & 18:04:05.17 & $-30: 09: 53.80$ & 16.34 & 1.876 & 2.07 & 1.3 & 4800 & -0.67 & 0.18 & - \\
\hline 0 & 95 & 554655 & 18:04:02.54 & $-30: 04: 31.00$ & 16.10 & 1.782 & 2.03 & 1.5 & 4900 & -0.34 & 0.17 & - \\
\hline 0 & 96 & 392918 & 18:03:36.89 & $-30: 07: 04.30$ & 16.37 & 2.017 & 1.97 & 1.4 & 4600 & 0.05 & 0.24 & - \\
\hline 0 & 97 & 63839 & 18:04:11.86 & $-30: 06: 20.80$ & 16.29 & 2.072 & 1.74 & 1.4 & 4300 & -0.22 & 0.29 & - \\
\hline 0 & 98 & 554700 & 18:03:58.14 & $-30: 02: 33.50$ & 16.07 & 1.637 & 2.02 & 1.4 & 4900 & -0.17 & 0.17 & - \\
\hline 0 & 99 & 554787 & 18:04:00.20 & $-30: 04: 06.70$ & 16.39 & 1.754 & 2.04 & 1.2 & 4700 & -0.58 & 0.16 & - \\
\hline 0 & 100 & 63855 & 18:04:16.04 & $-30: 05: 25.00$ & 16.33 & 2.029 & 1.67 & 1.4 & 4200 & 0.40 & 0.28 & - \\
\hline 1 & 101 & 63850 & 18:04:18.50 & $-30: 05: 40.30$ & 15.91 & 1.659 & 1.78 & 1.6 & 4600 & -1.61 & 0.18 & - \\
\hline 0 & 102 & 402294 & 18:03:51.01 & $-30: 04: 48.60$ & 16.29 & 1.761 & 2.05 & 1.2 & 4800 & -0.50 & 0.18 & - \\
\hline 0 & 103 & 63820 & 18:04:07.86 & $-30: 07: 35.00$ & 16.22 & 1.679 & 2.19 & 1.2 & 5100 & -0.14 & 0.20 & - \\
\hline 0 & 104 & 393015 & 18:03:52.55 & $-30: 07: 31.20$ & 16.31 & 1.775 & 2.09 & 1.3 & 4850 & -0.06 & 0.17 & - \\
\hline 0 & 105 & 554663 & 18:03:57.10 & $-30: 04: 13.10$ & 15.95 & 1.738 & 1.86 & 1.3 & 4700 & -0.72 & 0.14 & - \\
\hline 0 & 106 & 63834 & 18:04:14.43 & $-30: 06: 46.70$ & 16.14 & 1.802 & 2.08 & 1.4 & 4950 & 0.16 & 0.19 & - \\
\hline 2 & 107 & 402361 & 18:03:36.59 & $-30: 02: 16.10$ & 15.98 & 1.664 & 2.00 & 1.4 & 4950 & -1.05 & 0.21 & NGC 6522 \\
\hline 1 & 109 & 402307 & 18:03:4 & $-30: 04: 06.90$ & 16.28 & 1.835 & 1.93 & 1.5 & 4600 & 0.40 & 0.32 & - \\
\hline 0 & 110 & 402414 & 18:03: & $-30: 04: 41.80$ & 16.35 & 1.858 & 1.99 & 1.4 & 4650 & -0.21 & 0.26 & - \\
\hline 1 & 111 & 545288 & 18:04:02.82 & $-30: 05: 06.00$ & 16.31 & 1.791 & 1.94 & 1.3 & 4600 & 0.13 & 0.27 & - \\
\hline 0 & 112 & 554889 & 18:04:03.56 & $-30: 02: 34.00$ & 16.33 & 1.638 & 2.18 & 1.3 & 5000 & -0.10 & 0.18 & - \\
\hline 0 & 113 & 402315 & 18:03:40.66 & $-30: 03: 50.20$ & 16.14 & 1.862 & 1.97 & 1.4 & 4750 & -0.17 & 0.17 & - \\
\hline 0 & 114 & 554811 & 18:03:55.78 & $-30: 03: 48.80$ & 16.30 & 1.659 & 2.11 & 1.3 & 4900 & 0.17 & 0.18 & - \\
\hline 0 & 115 & 234671 & 18:03:34.49 & $-30: 07: 01.40$ & 16.27 & 1.976 & 1.86 & 1.4 & 4500 & 0.06 & 0.29 & - \\
\hline 0 & 117 & 402332 & 18:03:41.82 & $-30: 03: 24.30$ & 16.16 & 1.886 & 1.82 & 1.4 & 4500 & -0.31 & 0.19 & - \\
\hline 1 & 118 & 402322 & 18:03:42.24 & $-30: 03: 39.90$ & 16.01 & 1.701 & 1.94 & 1.5 & 4800 & -0.94 & 0.17 & NGC 6522 \\
\hline 0 & 119 & 564743 & 18:04:00.41 & $-30: 01: 12.00$ & 16.30 & 1.955 & 1.70 & 1.4 & 4250 & 0.21 & 0.31 & - \\
\hline 0 & 120 & 402311 & 18:03:37.88 & $-30: 03: 58.70$ & 16.34 & 1.888 & 1.89 & 1.5 & 4500 & 0.08 & 0.25 & - \\
\hline 1 & 122 & 4582 & $18: 0$ & $-30: 0$ & 16.00 & 1.720 & 2.01 & 1.3 & 4950 & -0.81 & 0.14 & NGC 6522 \\
\hline 0 & 123 & 4504 & 18:03:27.60 & $-30: 04: 29.40$ & 16.10 & 1.877 & 1.83 & 1.4 & 4550 & -0.25 & 0.21 & - \\
\hline 0 & 128 & 2607 & $18: 03: 4$ & $-30: 0$ & 16.26 & 1.707 & 2.04 & 1.3 & 4800 & -0 & 0.17 & NGC 6522 \\
\hline 1 & 130 & 402531 & 18:03: & $-30: 03: 03.00$ & 16.30 & 1.660 & 2.21 & 1.2 & 5100 & -0.8 & 0.17 & NGC 6522 \\
\hline 0 & 132 & 402325 & 18:03: & $-30: 03: 38.20$ & 16.27 & 1.876 & 1.87 & 1.4 & 4500 & -0.32 & 0.17 & - \\
\hline 0 & 135 & 256308 & $18: 03: 3$ & $-30: 00: 05.30$ & 16.06 & 1.623 & 1.95 & 1.4 & 4800 & -1.69 & 0.20 & - \\
\hline 0 & 3 & 585982 & 18:03:53.07 & $-29: 53: 30.50$ & 16.43 & 1.903 & 1.99 & 1.4 & 4600 & -0.08 & 0.26 & - \\
\hline 1 & 4 & 575308 & 18:03:56.30 & $-29: 56: 40.60$ & 16.47 & 2.255 & 1.84 & 1.4 & 4350 & 0.27 & 0.40 & - \\
\hline 0 & 5 & 575289 & 18:03:56.86 & $-29: 57: 26.20$ & 16.48 & 1.963 & 1.92 & 1.5 & 4450 & -0.50 & 0.24 & - \\
\hline 2 & 7 & 423298 & 18:03:51.29 & $-29: 57: 27.90$ & 16.54 & 2.192 & 1.91 & 1.2 & 4400 & -0.08 & 0.31 & - \\
\hline 1 & 8 & 433830 & 18:03:41.76 & $-29: 53: 17.60$ & 16.82 & 2.187 & 1.87 & 1.5 & 4200 & 0.17 & 0.43 & - \\
\hline 1 & 9 & 564963 & 18:03:59.09 & $-29: 59: 46.20$ & 16.62 & 1.971 & 1.83 & 1.0 & 4250 & 0.34 & 0.42 & - \\
\hline 0 & 10 & 554980 & 18:04:02.87 & $-30: 01: 29.20$ & 16.42 & 1.726 & 1.99 & 1.5 & 4600 & 0.31 & 0.39 & - \\
\hline 2 & 13 & 423304 & 18:03:43.75 & $-29: 57: 15.90$ & 16.95 & 2.296 & 2.03 & 1.4 & 4350 & 0.22 & 0.42 & - \\
\hline 2 & 14 & 102833 & 18:04:14.55 & $-29: 51: 36.90$ & 16.75 & 2.108 & 2.05 & 1.5 & 4500 & 0.29 & 0.37 & - \\
\hline 1 & 15 & 102853 & 18:04:11.78 & $-29: 51: 09.50$ & 16.43 & 2.028 & 1.86 & 1.2 & 4400 & 0.15 & 0.39 & - \\
\hline 0 & 16 & 564768 & 18:04:05.90 & $-29: 59: 53.10$ & 16.59 & 2.186 & 1.74 & 1.3 & 4150 & -0.30 & 0.26 & - \\
\hline 0 & 17 & 586077 & 18:04:06.36 & $-29: 53: 53.20$ & 16.66 & 2.002 & 2.02 & 1.3 & 4500 & 0.21 & 0.28 & - \\
\hline 1 & 18 & 586005 & 18:04:04.92 & $-29: 52: 42.30$ & 16.55 & 2.133 & 1.91 & 1.3 & 4400 & 0.29 & 0.42 & - \\
\hline 0 & 19 & 564789 & 18:04:03.96 & $-29: 59: 21.90$ & 16.60 & 2.255 & 1.70 & 1.2 & 4100 & -0.15 & 0.28 & - \\
\hline 2 & 20 & 596502 & 18:03:59.27 & $-29: 49: 51.50$ & 16.94 & 2.520 & 1.87 & 1.1 & 4150 & 0.28 & 0.50 & - \\
\hline 1 & 21 & 575360 & 18:04:00.11 & $-29: 55: 07.30$ & 16.56 & 2.077 & 1.96 & 1.2 & 4500 & -0.05 & 0.37 & - \\
\hline 1 & 22 & 564991 & 18:04:02.46 & $-29: 59: 28.90$ & 16.73 & 2.117 & 1.98 & 1.4 & 4400 & 0.19 & 0.34 & - \\
\hline 2 & 26 & 82760 & 18:04:13.27 & $-29: 58: 17.80$ & 16.64 & 2.192 & 1.87 & 1.5 & 4300 & 0.25 & 0.41 & - \\
\hline 1 & 28 & 82727 & 18:04:08.96 & $-29: 59: 41.20$ & 16.59 & 2.234 & 1.78 & 1.3 & 4200 & 0.17 & 0.35 & - \\
\hline 0 & 29 & 92557 & 18:04:12.49 & $-29: 57: 16.00$ & 16.43 & 1.888 & 2.05 & 1.4 & 4700 & -0.07 & 0.27 & - \\
\hline 2 & 33 & 231128 & 18:04:31.51 & $-29: 59: 51.80$ & 16.55 & 2.338 & 1.76 & 1.4 & 4200 & 0.17 & 0.45 & - \\
\hline
\end{tabular}


Table 3. continued.

\begin{tabular}{|c|c|c|c|c|c|c|c|c|c|c|c|c|}
\hline $\mathrm{QF}^{a}$ & ID & OGLE-ID & RA & Dec & $V$ & $V-I$ & $\log g$ & $V_{\mathrm{t}}$ & $T_{\text {eff }}$ & {$[\mathrm{Fe} / \mathrm{H}]$} & $\sigma^{b}$ & Cluster? \\
\hline 0 & 34 & 82717 & 18:04:17.68 & $-30: 00: 29.70$ & 16.57 & 2.286 & 1.77 & 1.1 & 4150 & 0.28 & 0.39 & - \\
\hline 1 & 35 & 240216 & 18:04:26.41 & $-29: 57: 16.50$ & 16.42 & 1.968 & 1.86 & 1.2 & 4400 & 0.19 & 0.39 & - \\
\hline 0 & 36 & 240459 & 18:04:33.58 & $-29: 54: 49.40$ & 16.57 & 1.880 & 2.02 & 1.2 & 4550 & -0.39 & 0.25 & - \\
\hline 1 & 37 & 240394 & 18:04:34.29 & $-29: 55: 54.40$ & 16.56 & 1.960 & 2.04 & 1.6 & 4600 & 0.14 & 0.30 & - \\
\hline 1 & 38 & 231369 & 18:04:33.83 & $-29: 58: 19.60$ & 16.71 & 2.112 & 1.82 & 1.5 & 4200 & 0.29 & 0.39 & - \\
\hline 0 & 40 & 231367 & 18:04:26.86 & $-29: 58: 20.80$ & 16.45 & 1.849 & 2.00 & 1.4 & 4600 & -0.01 & 0.25 & - \\
\hline 1 & 41 & 231310 & 18:04:33.50 & $-29: 59: 12.50$ & 16.50 & 1.924 & 1.95 & 1.1 & 4500 & 0.30 & 0.38 & - \\
\hline 1 & 42 & 374186 & 18:04:41.73 & $-29: 55: 17.60$ & 16.76 & 2.142 & 1.96 & 1.2 & 4350 & 0.20 & 0.36 & - \\
\hline 1 & 43 & 231325 & $18: 04: 24.08$ & $-29: 59: 00.60$ & 16.69 & 2.078 & 1.81 & 1.5 & 4200 & 0.34 & 0.36 & - \\
\hline 0 & 46 & 222627 & $18: 04: 25.51$ & $-30: 01: 60.00$ & 16.81 & 2.270 & 1.78 & 1.1 & 4100 & 0.13 & 0.38 & - \\
\hline 0 & 49 & 231185 & 18:04:33.44 & $-30: 01: 12.90$ & 16.57 & 1.997 & 1.98 & 1.3 & 4500 & 0.19 & 0.32 & - \\
\hline 2 & 51 & 231233 & 18:04:34.84 & $-30: 00: 26.40$ & 16.45 & 1.819 & 1.97 & 1.3 & 4550 & -0.16 & 0.28 & - \\
\hline 0 & 53 & 365797 & 18:04:54.55 & $-29: 58: 16.40$ & 16.40 & 2.017 & 1.95 & 1.3 & 4550 & -0.19 & 0.25 & - \\
\hline 0 & 54 & 222412 & 18:04:29.91 & $-30: 04: 32.00$ & 16.42 & 2.074 & 1.86 & 1.3 & 4400 & -0.11 & 0.21 & - \\
\hline 1 & 55 & 222408 & 18:04:29.73 & $-30: 04: 49.60$ & 16.65 & 2.182 & 1.72 & 1.5 & 4100 & 0.31 & 0.36 & - \\
\hline 1 & 56 & 357466 & 18:04:55.47 & $-30: 03: 28.00$ & 16.92 & 2.435 & 1.98 & 1.3 & 4300 & 0.43 & 0.44 & - \\
\hline 0 & 57 & 350527 & $18: 04: 47.62$ & $-30: 05: 14.10$ & 16.55 & 2.234 & 2.04 & 1.4 & 4600 & -0.65 & 0.16 & - \\
\hline 0 & 59 & 222523 & 18:04:32.75 & $-30: 03: 53.10$ & 16.74 & 2.191 & 1.88 & 1.4 & 4250 & 0.01 & 0.34 & - \\
\hline 1 & 60 & 222543 & 18:04:38.20 & $-30: 03: 24.50$ & 16.85 & 2.452 & 1.99 & 1.1 & 4350 & 0.34 & 0.41 & - \\
\hline 0 & 61 & 357436 & 18:04:40.16 & $-30: 03: 53.10$ & 16.50 & 1.913 & 1.95 & 1.3 & 4500 & 0.44 & 0.42 & - \\
\hline 0 & 62 & 357459 & 18:04:47.78 & $-30: 03: 32.10$ & 16.64 & 2.008 & 2.08 & 1.5 & 4600 & -0.01 & 0.29 & NGC 6528 \\
\hline 0 & 64 & 73607 & 18:04:13.37 & $-30: 03: 40.10$ & 16.44 & 1.772 & 2.00 & 1.2 & 4600 & 0.28 & 0.40 & - \\
\hline 0 & 65 & 73483 & 18:04:10.39 & $-30: 03: 21.30$ & 16.63 & 2.142 & 1.87 & 1.4 & 4300 & 0.16 & 0.33 & - \\
\hline 1 & 66 & 73609 & 18:04:14.37 & $-30: 03: 35.30$ & 16.73 & 2.210 & 1.91 & 1.5 & 4300 & 0.28 & 0.49 & - \\
\hline 0 & 67 & 214192 & 18:04:23.95 & $-30: 05: 57.80$ & 16.58 & 1.904 & 1.96 & 1.3 & 4450 & 0.11 & 0.29 & - \\
\hline 1 & 69 & 73472 & 18:04:22.33 & $-30: 04: 15.50$ & 16.73 & 2.193 & 1.98 & 1.5 & 4400 & 0.49 & 0.48 & - \\
\hline 0 & 70 & 64005 & 18:04:21.76 & $-30: 06: 12.00$ & 16.58 & 1.959 & 1.99 & 1.4 & 4500 & -0.25 & 0.19 & - \\
\hline 0 & 71 & 205265 & 18:04:33.17 & $-30: 09: 49.70$ & 16.45 & 2.155 & 1.94 & 1.3 & 4500 & 0.02 & 0.32 & - \\
\hline 1 & 72 & 214042 & 18:04:29.19 & $-30: 06: 11.80$ & 16.65 & 2.256 & 1.76 & 1.3 & 4150 & 0.38 & 0.39 & - \\
\hline 0 & 73 & 350483 & 18:04:48.98 & $-30: 08: 07.20$ & 16.83 & 2.511 & 1.87 & 1.3 & 4200 & 0.14 & 0.39 & - \\
\hline 0 & 74 & 64018 & 18:04:22.49 & $-30: 06: 04.50$ & 16.42 & 1.777 & 1.92 & 1.5 & 4500 & 0.15 & 0.33 & - \\
\hline 0 & 75 & 63859 & 18:04:13.08 & $-30: 05: 10.80$ & 16.43 & 1.931 & 1.86 & 1.5 & 4400 & 0.25 & 0.33 & - \\
\hline 0 & 76 & 545445 & 18:04:06.17 & $-30: 05: 55.20$ & 16.56 & 1.955 & 2.01 & 1.5 & 4550 & 0.13 & 0.29 & - \\
\hline 0 & 77 & 63840 & 18:04:08.08 & $-30: 06: 19.40$ & 16.59 & 1.950 & 1.99 & 1.1 & 4500 & 0.31 & 0.41 & - \\
\hline 1 & 78 & 54108 & 18:04:11.63 & $-30: 09: 49.10$ & 16.55 & 2.193 & 1.91 & 1.5 & 4400 & 0.46 & 0.42 & - \\
\hline 1 & 79 & 54125 & 18:04:12.91 & $-30: 09: 05.60$ & 16.77 & 2.335 & 2.00 & 1.3 & 4400 & 0.07 & 0.36 & - \\
\hline 1 & 80 & 73467 & 18:04:08.39 & $-30: 04: 37.80$ & 17.04 & 2.544 & 2.00 & 1.4 & 4250 & 0.12 & 0.35 & - \\
\hline 1 & 81 & 54133 & 18:04:15.65 & $-30: 08: 53.50$ & 16.64 & 2.156 & 1.67 & 1.0 & 4050 & 0.35 & 0.44 & - \\
\hline 0 & 82 & 54078 & $18: 04: 22.03$ & $-30: 11: 20.90$ & 16.59 & 2.113 & 1.89 & 1.5 & 4350 & 0.09 & 0.35 & - \\
\hline 2 & 83 & 63829 & 18:04:15.01 & $-30: 07: 08.60$ & 16.79 & 2.231 & 2.01 & 1.5 & 4400 & -0.01 & 0.40 & - \\
\hline 0 & 85 & 537095 & 18:04:03.45 & $-30: 09: 57.60$ & 16.45 & 1.824 & 1.93 & 1.2 & 4500 & 0.31 & 0.32 & - \\
\hline 0 & 86 & 545222 & 18:03:57.53 & $-30: 08: 21.20$ & 16.41 & 2.079 & 1.78 & 1.4 & 4300 & 0.16 & 0.36 & - \\
\hline 0 & 87 & 545438 & 18:04:02.43 & $-30: 06: 05.60$ & 16.80 & 2.147 & 1.97 & 1.5 & 4350 & 0.12 & 0.28 & - \\
\hline 0 & 88 & 545233 & 18:03:59.73 & $-30: 07: 46.90$ & 16.56 & 2.256 & 1.88 & 1.3 & 4350 & 0.31 & 0.41 & - \\
\hline 2 & 91 & 545313 & 18:03:56.58 & $-30: 08: 06.00$ & 16.90 & 2.289 & 2.05 & 1.4 & 4400 & 0.19 & 0.30 & - \\
\hline 0 & 92 & 537092 & 18:04:06.28 & $-30: 09: 59.40$ & 16.53 & 1.956 & 2.03 & 1.0 & 4600 & -0.25 & 0.20 & - \\
\hline 2 & 93 & 545277 & 18:04:05.34 & $-30: 05: 52.50$ & 16.95 & 2.530 & 1.84 & 1.2 & 4100 & 0.35 & 0.46 & - \\
\hline 1 & 95 & 402415 & 18:03:49.43 & $-30: 04: 39.30$ & 16.65 & 2.042 & 2.08 & 1.2 & 4600 & 0.01 & 0.28 & - \\
\hline 0 & 96 & 554670 & 18:03:56.05 & $-30: 03: 54.10$ & 16.62 & 2.248 & 1.75 & 1.3 & 4150 & -0.26 & 0.26 & - \\
\hline 1 & 97 & 554748 & 18:03:55.84 & $-30: 04: 35.30$ & 16.42 & 1.798 & 1.99 & 1.3 & 4600 & 0.39 & 0.49 & - \\
\hline 0 & 98 & 392952 & 18:03:49.50 & $-30: 05: 28.30$ & 16.61 & 2.196 & 1.79 & 1.5 & 4200 & 0.13 & 0.31 & - \\
\hline 1 & 99 & 392896 & 18:03:41.61 & $-30: 07: 54.60$ & 16.64 & 2.407 & 1.80 & 1.3 & 4200 & -0.12 & 0.36 & - \\
\hline 0 & 100 & 393083 & 18:03:49.11 & $-30: 06: 13.80$ & 16.65 & 1.974 & 1.98 & 1.5 & 4450 & 0.03 & 0.25 & - \\
\hline 0 & 101 & 393053 & 18:03:48.01 & $-30: 06: 51.10$ & 16.63 & 2.009 & 1.83 & 1.2 & 4250 & 0.49 & 0.47 & - \\
\hline 0 & 102 & 392931 & 18:03:51.84 & $-30: 06: 27.90$ & 16.42 & 1.990 & 1.89 & 1.5 & 4450 & -0.04 & 0.27 & - \\
\hline 0 & 103 & 545269 & 18:03:53.81 & $-30: 06: 15.80$ & 16.63 & 2.225 & 1.83 & 1.1 & 4250 & 0.45 & 0.40 & - \\
\hline 0 & 104 & 554683 & 18:04:04.81 & $-30: 03: 00.00$ & 16.61 & 2.032 & 2.00 & 1.2 & 4500 & -0.20 & 0.25 & - \\
\hline 0 & 105 & 554668 & 18:04:00.27 & $-30: 04: 00.60$ & 16.58 & 2.157 & 1.85 & 1.3 & 4300 & 0.08 & 0.35 & - \\
\hline 2 & 107 & 78106 & 18:03:19.96 & $-30: 04: 27.00$ & 16.89 & 2.398 & 1.98 & 1.2 & 4300 & -0.17 & 0.35 & - \\
\hline 0 & 108 & 402498 & 18:03:40.67 & $-30: 03: 27.80$ & 16.46 & 1.857 & 1.90 & 1.2 & 4450 & 0.55 & 0.42 & - \\
\hline 1 & 109 & 234704 & 18:03:25.02 & $-30: 05: 01.50$ & 16.42 & 1.936 & 1.93 & 1.4 & 4500 & -0.18 & 0.28 & - \\
\hline 0 & 110 & 67494 & 18:03:16.82 & $-30: 06: 10.80$ & 16.43 & 1.959 & 2.02 & 1.1 & 4650 & -0.05 & 0.25 & - \\
\hline 0 & 111 & 234701 & 18:03:33.22 & $-30: 05: 08.40$ & 16.56 & 2.027 & 1.98 & 1.2 & 4500 & 0.12 & 0.38 & - \\
\hline
\end{tabular}


Table 3. continued.

\begin{tabular}{|c|c|c|c|c|c|c|c|c|c|c|c|c|}
\hline $\mathrm{QF}^{a}$ & ID & OGLE-ID & RA & Dec & $V$ & $V-I$ & $\log g$ & $V_{\mathrm{t}}$ & $T_{\text {eff }}$ & {$[\mathrm{Fe} / \mathrm{H}]$} & $\sigma^{b}$ & Cluster? \\
\hline 1 & 112 & 234888 & $18: 03: 32.84$ & $-30: 05: 44.60$ & 17.00 & 2.384 & 1.94 & 1.5 & 4200 & 0.28 & 0.45 & - \\
\hline 1 & 113 & 554713 & 18:03:56.58 & $-30: 01: 55.50$ & 16.81 & 2.387 & 1.90 & 1.4 & 4250 & 0.20 & 0.41 & - \\
\hline 0 & 114 & 554956 & 18:04:01.61 & $-30: 01: 49.20$ & 16.43 & 1.954 & 1.99 & 1.1 & 4600 & -0.01 & 0.27 & - \\
\hline 1 & 115 & 392951 & 18:03:42.68 & $-30: 05: 29.80$ & 16.63 & 2.010 & 2.10 & 1.3 & 4650 & 0.10 & 0.34 & - \\
\hline 0 & 116 & 412750 & 18:03:39.50 & $-30: 00: 53.80$ & 16.45 & 1.946 & 1.83 & 1.1 & 4350 & 0.11 & 0.28 & - \\
\hline 0 & 117 & 411479 & 18:03:36.82 & $-30: 01: 46.90$ & 16.43 & 1.919 & 2.32 & 1.2 & 5200 & -0.30 & 0.18 & - \\
\hline 0 & 118 & 402656 & 18:03:38.71 & $-30: 01: 30.20$ & 16.42 & 1.805 & 2.08 & 1.2 & 4750 & -0.32 & 0.25 & - \\
\hline 1 & 119 & 554694 & 18:04:04.57 & $-30: 02: 39.60$ & 16.69 & 2.446 & 1.89 & 1.2 & 4300 & 0.15 & 0.47 & - \\
\hline 0 & 120 & 402375 & 18:03:45.77 & $-30: 02: 02.30$ & 16.65 & 2.228 & 1.80 & 1.4 & 4200 & 0.05 & 0.35 & - \\
\hline 0 & 121 & 244829 & 18:03:36.41 & $-30: 02: 19.80$ & 16.40 & 1.724 & 2.09 & 1.4 & 4800 & -1.09 & 0.18 & NGC 6522 \\
\hline 2 & 122 & 402353 & $18: 03: 42.24$ & $-30: 02: 38$ & 16.84 & 2.624 & 2.27 & 1.5 & 4800 & 0.01 & 0.42 & - \\
\hline 0 & 123 & 244738 & $18: 03: 27.62$ & $-30: 03: 11.40$ & 16.45 & 1.788 & 2.12 & 1.3 & 4800 & -0.25 & 0.22 & - \\
\hline 0 & 124 & 402347 & 18:03:44.54 & $-30: 02: 52.90$ & 16.71 & 2.395 & 1.82 & 1.2 & 4200 & 0.19 & 0.45 & - \\
\hline 0 & 126 & 564772 & 18:03:53.92 & $-29: 59: 44.20$ & 16.75 & 2.542 & 1.92 & 1.5 & 4300 & -0.07 & 0.44 & - \\
\hline 1 & 128 & 423286 & 18:03:40.01 & $-29: 57: 54.80$ & 16.62 & 2.130 & 1.90 & 1.2 & 4350 & 0.05 & 0.33 & - \\
\hline 0 & 129 & 267974 & 18:03:24.89 & $-29: 55: 55.10$ & 16.63 & 2.057 & 1.94 & 1.2 & 4400 & 0.31 & 0.39 & - \\
\hline 0 & 133 & 412753 & 18:03:52.50 & $-30: 00: 50.90$ & 16.57 & 2.136 & 1.84 & 1.1 & 4300 & 0.33 & 0.35 & - \\
\hline 1 & 134 & 256345 & $18: 03: 23.54$ & $-29: 58: 29.50$ & 16.50 & 2.057 & 1.78 & 1.5 & 4250 & 0.33 & 0.44 & - \\
\hline \multicolumn{13}{|c|}{ Field at $b=-6^{\circ}$} \\
\hline 0 & 2 & $41958 \mathrm{c} 3$ & 18:10:11.78 & $-31: 39: 03.30$ & 15.64 & 1.506 & 2.04 & 1.5 & 5100 & 0.05 & 0.19 & - \\
\hline 0 & 3 & $157820 \mathrm{c} 3$ & 18:10:04.89 & $-31: 36: 45.30$ & 15.63 & 1.588 & 1.87 & 1.6 & 4800 & -0.73 & 0.13 & - \\
\hline 0 & 4 & $32799 \mathrm{c} 3$ & 18:10:09.69 & $-31: 40: 21.90$ & 15.99 & 1.546 & 2.04 & 1.5 & 4850 & -1.25 & 0.19 & - \\
\hline 0 & 5 & $76187 \mathrm{c} 3$ & 18:09:56.25 & $-31: 34: 12.70$ & 15.79 & 1.771 & 1.79 & 1.6 & 4550 & -0.42 & 0.16 & - \\
\hline 0 & 6 & $38354 \mathrm{c} 3$ & 18:10:04.92 & $-31: 39: 34.00$ & 15.67 & 1.671 & 1.83 & 1.7 & 4700 & -0.61 & 0.13 & - \\
\hline 0 & 7 & $203158 \mathrm{c} 3$ & 18:10:14.94 & $-31: 40: 51.40$ & 15.58 & 1.578 & 1.86 & 1.6 & 4800 & -0.04 & 0.13 & - \\
\hline 0 & 8 & $39802 \mathrm{c} 3$ & 18:10:00.70 & $-31: 39: 21.50$ & 15.85 & 1.413 & 2.17 & 1.6 & 5200 & -0.50 & 0.16 & - \\
\hline 0 & 9 & $43054 c 3$ & $18: 10: 01.23$ & $-31: 38: 5$ & 15.98 & 1.542 & 2.01 & 1.5 & 4800 & -1.03 & 0.15 & - \\
\hline 0 & 10 & $46885 \mathrm{c} 3$ & 18:09:55.24 & $-31: 38:$ & 15.89 & 1.843 & 1.70 & 1.5 & 4350 & 0.00 & 0.18 & - \\
\hline 0 & 11 & $1604 c 2$ & 18:10:19.01 & $-31: 44: 50.60$ & 15.90 & 1.627 & 1.92 & 1.4 & 4700 & -1.13 & 0.16 & NGC 6558 \\
\hline 0 & 12 & $36989 \mathrm{c} 3$ & 18:09:54.14 & $-31: 39: 46.00$ & 15.78 & 1.658 & 1.88 & 1.5 & 4700 & 0.05 & 0.17 & - \\
\hline 0 & 13 & $36067 \mathrm{c} 3$ & 18:09:53.51 & $-31: 39: 53.70$ & 15.77 & 1.731 & 1.78 & 1.4 & 4550 & 0.08 & 0.21 & - \\
\hline 0 & 15 & $77454 c 2$ & $18: 10: 24.40$ & $-31: 33: 39.80$ & 15.60 & 1.557 & 1.95 & 1.6 & 4950 & -0.38 & 0.13 & - \\
\hline 0 & 16 & $43562 \mathrm{c} 2$ & 18:10:20.85 & $-31: 38: 37.90$ & 15.84 & 1.702 & 1.84 & 1.7 & 4600 & -0.81 & 0.14 & - \\
\hline 0 & 17 & $32832 \mathrm{c} 2$ & 18:10:18.07 & $-31: 40: 13.80$ & 15.92 & 1.777 & 1.71 & 1.5 & 4350 & -0.03 & 0.19 & - \\
\hline 0 & 18 & $62009 \mathrm{c} 2$ & 18:10:16.95 & $-31: 35: 55.30$ & 15.96 & 1.734 & 1.73 & 1.5 & 4350 & -0.39 & 0.11 & - \\
\hline 0 & 19 & $38565 \mathrm{c} 2$ & 18:10:15.97 & $-31: 39: 22.80$ & 15.90 & 1.723 & 1.87 & 1.7 & 4600 & -0.26 & 0.11 & - \\
\hline 0 & 20 & $204270 \mathrm{c} 3$ & 18:10:14.79 & $-31: 39: 30.30$ & 15.93 & 1.571 & 2.05 & 1.3 & 4900 & 0.02 & 0.13 & - \\
\hline 0 & 21 & $429 \mathrm{c} 3$ & 18:10:10.00 & $-31: 3$ & 15.86 & 1.747 & 1.79 & 1.5 & 4500 & -0.76 & 0.12 & - \\
\hline 0 & 22 & $671 \mathrm{c} 3$ & $18: 10: 10.33$ & $-31: 36: 57.50$ & 15.76 & 1.576 & 1.98 & 1.3 & 4900 & -0.20 & 0.12 & - \\
\hline 0 & 23 & $213 \mathrm{c} 2$ & 18:10:31.18 & $-31: 4$ & 15.92 & 1.604 & 1.87 & 1.5 & 4600 & 0.09 & 0.17 & - \\
\hline 0 & 24 & c2 2 & 18:10:33.48 & $-31: 39: 51.20$ & 15.76 & 1.506 & 1.92 & 1.6 & 4800 & -1.16 & 0.17 & - \\
\hline 0 & 26 & $31338 \mathrm{c} 2$ & $18: 10: 29.03$ & $-31: 4$ & 15.80 & 1.669 & 1.89 & 1.6 & 4700 & -0.55 & 0.14 & - \\
\hline 0 & 28 & $53477 \mathrm{c} 2$ & 18:10:32.42 & $-31: 37: 09.60$ & 15.77 & 1.686 & 1.85 & 1.6 & 4650 & -0.55 & 0.14 & - \\
\hline 0 & 29 & $56410 \mathrm{c} 2$ & 18:10:30.35 & $-31: 36: 44.00$ & 15.77 & 1.680 & 1.81 & 1.5 & 4600 & -1.10 & 0.13 & - \\
\hline 0 & 30 & $4799 \mathrm{c} 2$ & 18:10:20.10 & $-31: 44: 21.90$ & 15.98 & 1.521 & 2.10 & 1.2 & 4950 & -0.12 & 0.13 & - \\
\hline 2 & 31 & $43239 \mathrm{c} 2$ & $18: 10: 25.53$ & $-31: 38: 40.80$ & 15.93 & 1.459 & 2.19 & 1.6 & 5200 & -1.26 & 0.16 & - \\
\hline 0 & 33 & $14297 \mathrm{c} 2$ & 18:10:50.22 & $-31: 42: 57.00$ & 15.84 & 1.516 & 2.01 & 1.8 & 4900 & -0.66 & 0.13 & - \\
\hline 0 & 34 & $17437 \mathrm{c} 2$ & 18:10:41.67 & $-31: 42: 29.20$ & 15.87 & 1.616 & 1.97 & 1.6 & 4800 & -0.50 & 0.13 & - \\
\hline 1 & 35 & $41995 \mathrm{c} 2$ & $18: 10: 45.45$ & $-31: 38: 51.60$ & 15.91 & 1.533 & 1.98 & 2.0 & 4800 & -1.58 & 0.16 & - \\
\hline 0 & 36 & $30173 c 2$ & 18:10:35.61 & $-31: 40: 37.30$ & 15.93 & 1.483 & 2.05 & 1.7 & 4900 & -0.90 & 0.18 & - \\
\hline 0 & 37 & $45160 \mathrm{c} 2$ & 18:10:52.14 & $-31: 38: 23.20$ & 15.87 & 1.722 & 1.91 & 1.5 & 4700 & -0.56 & 0.12 & - \\
\hline 0 & 38 & $13661 \mathrm{c} 2$ & 18:10:35.13 & $-31: 43: 02.70$ & 15.81 & 1.546 & 1.98 & 1.5 & 4850 & -0.09 & 0.11 & - \\
\hline 0 & 39 & $212324 \mathrm{c} 6$ & 18:10:26.03 & $-31: 45: 13.20$ & 15.65 & 1.548 & 1.88 & 1.2 & 4800 & -0.32 & 0.18 & - \\
\hline 0 & 40 & $10381 \mathrm{c} 2$ & 18:10:27.82 & $-31: 43: 32.10$ & 15.84 & 1.552 & 1.90 & 1.3 & 4700 & -0.14 & 0.16 & - \\
\hline 0 & 41 & $14893 \mathrm{c} 2$ & 18:10:42.48 & $-31: 42: 51.80$ & 15.99 & 1.870 & 1.51 & 1.5 & 4050 & -0.47 & 0.16 & - \\
\hline 0 & 42 & $204828 \mathrm{c} 2$ & $18: 10: 53.24$ & $-31: 38: 26.70$ & 15.91 & 1.539 & 2.10 & 1.5 & 5000 & -0.22 & 0.15 & - \\
\hline 0 & 43 & $203913 \mathrm{c} 2$ & 18:10:54.62 & $-31: 39: 38.00$ & 15.63 & 1.550 & 1.93 & 1.5 & 4900 & -0.24 & 0.12 & - \\
\hline 0 & 44 & $33058 \mathrm{c} 2$ & 18:10:41.51 & $-31: 40: 11.80$ & 15.90 & 1.732 & 1.84 & 1.7 & 4550 & -0.35 & 0.17 & - \\
\hline 0 & 45 & $212175 \mathrm{c} 6$ & 18:10:53.08 & $-31: 45: 21.60$ & 15.90 & 1.779 & 1.90 & 1.5 & 4650 & -0.47 & 0.14 & - \\
\hline 0 & 46 & $213150 \mathrm{c} 6$ & $18: 10: 47.52$ & $-31: 45: 05.30$ & 15.92 & 1.895 & 1.67 & 1.5 & 4300 & -0.02 & 0.17 & - \\
\hline 0 & 48 & $1678 \mathrm{c} 2$ & $18: 10: 40.43$ & $-31: 44: 49.80$ & 15.76 & 1.512 & 1.98 & 1.8 & 4900 & -0.95 & 0.13 & - \\
\hline 0 & 49 & $874 c 2$ & $18: 10: 45.83$ & $-31: 44: 56.60$ & 15.91 & 1.772 & 1.84 & 1.5 & 4550 & -0.32 & 0.13 & - \\
\hline 0 & 50 & $7694 c 2$ & 18:10:50.37 & $-31: 43: 55.60$ & 15.80 & 1.434 & 2.11 & 1.9 & 5100 & 0.15 & 0.19 & - \\
\hline
\end{tabular}


Table 3. continued.

\begin{tabular}{|c|c|c|c|c|c|c|c|c|c|c|c|c|}
\hline $\mathrm{QF}^{a}$ & ID & OGLE-ID & RA & Dec & $V$ & $V-I$ & $\log g$ & $V_{\mathrm{t}}$ & $T_{\text {eff }}$ & {$[\mathrm{Fe} / \mathrm{H}]$} & $\sigma^{b}$ & Cluster? \\
\hline 0 & 51 & $8312 \mathrm{c} 2$ & $18: 10: 39.22$ & $-31: 43: 50.30$ & 15.85 & 1.511 & 2.07 & 2.0 & 5000 & -0.32 & 0.13 & - \\
\hline 0 & 52 & $19402 \mathrm{c} 1$ & 18:11:13.14 & $-31: 42: 05.50$ & 15.87 & 1.739 & 1.83 & 1.5 & 4550 & -0.61 & 0.13 & - \\
\hline 0 & 53 & $23483 \mathrm{c} 1$ & 18:11:08.20 & $-31: 41: 28.70$ & 15.51 & 1.411 & 2.01 & 1.4 & 5150 & -0.52 & 0.12 & - \\
\hline 0 & 54 & $98692 \mathrm{c} 6$ & 18:10:15.50 & $-31: 46: 54.00$ & 15.99 & 1.498 & 2.22 & 1.4 & 5000 & 0.07 & 0.18 & - \\
\hline 0 & 55 & $94324 c 6$ & $18: 10: 37.62$ & $-31: 47: 33.00$ & 15.82 & 1.736 & 1.90 & 1.6 & 4700 & -0.39 & 0.13 & - \\
\hline 0 & 56 & $99147 \mathrm{c} 5$ & 18:11:07.80 & $-31: 46: 29.00$ & 15.96 & 1.742 & 1.86 & 1.6 & 4550 & -0.60 & 0.14 & - \\
\hline 0 & 58 & $96158 c 6$ & $18: 10: 45.14$ & $-31: 47: 16.80$ & 15.92 & 1.610 & 2.10 & 1.4 & 5000 & -0.37 & 0.17 & - \\
\hline 0 & 60 & $100047 \mathrm{c} 6$ & $18: 10: 52.30$ & $-31: 46: 42.10$ & 15.95 & 1.891 & 1.72 & 1.5 & 4350 & -0.33 & 0.14 & - \\
\hline 0 & 61 & $102180 \mathrm{c} 6$ & $18: 10: 36.37$ & $-31: 46: 23.50$ & 15.95 & 1.782 & 1.86 & 1.4 & 4550 & -0.39 & 0.14 & - \\
\hline 0 & 62 & $211484 c 6$ & $18: 10: 53.63$ & $-31: 46: 31.10$ & 15.75 & 1.683 & 1.92 & 1.4 & 4800 & -0.60 & 0.11 & - \\
\hline 0 & 64 & $106969 \mathrm{c} 6$ & $18: 10: 18.03$ & $-31: 45: 43.50$ & 15.70 & 1.473 & 1.98 & 1.5 & 4950 & -1.00 & 0.19 & NGC 6558 \\
\hline 0 & 65 & $91438 \mathrm{c} 6$ & 18:10:17.39 & $-31: 47: 58.40$ & 15.73 & 1.447 & 2.10 & 1.4 & 5150 & -0.62 & 0.12 & - \\
\hline 0 & 66 & $74262 c 6$ & 18:10:50.66 & $-31: 50: 30.70$ & 15.93 & 1.542 & 2.08 & 1.2 & 4950 & -0.32 & 0.16 & - \\
\hline 0 & 67 & $79869 \mathrm{c} 6$ & 18:10:31.64 & $-31: 49: 40.90$ & 15.88 & 1.495 & 2.23 & 1.4 & 5300 & -0.48 & 0.15 & - \\
\hline 0 & 69 & $98974 \mathrm{c} 6$ & 18:10:36.36 & $-31: 46: 51.60$ & 15.72 & 1.469 & 2.12 & 1.3 & 5200 & -0.39 & 0.18 & - \\
\hline 0 & 70 & $99069 c 6$ & $18: 10: 22.08$ & $-31: 46: 50.70$ & 15.86 & 1.418 & 2.27 & 1.6 & 5400 & -0.26 & 0.20 & - \\
\hline 0 & 72 & $100384 c 6$ & $18: 10: 25.39$ & $-31: 46: 39.10$ & 15.89 & 1.673 & 1.83 & 1.6 & 4550 & -0.57 & 0.12 & - \\
\hline 0 & 73 & $108928 \mathrm{c} 6$ & 18:10:21.50 & $-31: 45: 26.70$ & 15.79 & 1.552 & 1.96 & 1.5 & 4850 & -0.88 & 0.15 & NGC 6558 \\
\hline 0 & 74 & $101274 c 6$ & $18: 10: 27.26$ & $-31: 46: 31.50$ & 15.83 & 1.486 & 2.14 & 1.5 & 5150 & -0.06 & 0.11 & - \\
\hline 0 & 75 & $71769 \mathrm{c} 6$ & $18: 10: 32.70$ & $-31: 50: 52.80$ & 15.62 & 1.442 & 2.13 & 1.5 & 5300 & -0.22 & 0.19 & - \\
\hline 0 & 77 & $62520 c 6$ & 18:10:21.66 & $-31: 52: 14.40$ & 15.98 & 1.491 & 2.09 & 1.5 & 4950 & -0.84 & 0.19 & - \\
\hline 0 & 78 & $60577 \mathrm{c} 6$ & 18:10:24.24 & $-31: 52: 31.50$ & 15.73 & 1.573 & 1.97 & 1.5 & 4900 & -0.18 & 0.16 & - \\
\hline 0 & 79 & $43679 \mathrm{c} 6$ & $18: 10: 28.35$ & $-31: 55: 00.70$ & 15.99 & 1.510 & 2.10 & 1.4 & 4950 & -0.59 & 0.18 & - \\
\hline 0 & 80 & $55804 c 6$ & 18:10:27.09 & $-31: 53: 14.00$ & 15.99 & 1.854 & 1.54 & 1.5 & 4100 & 0.19 & 0.24 & - \\
\hline 0 & 81 & $54561 \mathrm{c} 6$ & $18: 10: 2$ & $-31: 53: 24.70$ & 15.73 & 1.442 & 1.97 & 1.4 & 4900 & -0.25 & 0.13 & - \\
\hline 0 & 82 & $80281 c 6$ & 18:10: & $-31: 49: 37.30$ & 15.98 & 1.490 & 2.15 & 1.5 & 5050 & -0.08 & 0.11 & - \\
\hline 0 & 83 & 6878 & $18: 10$ & $-31:$ & 15.83 & 1.718 & 1.93 & 1.5 & 4750 & -0.50 & 0.16 & - \\
\hline 0 & 84 & $66376 \mathrm{c} 6$ & $18: 10: 3$ & $-31: 51: 40.50$ & 15.82 & 1.536 & 1.87 & 1.5 & 4650 & 0.19 & 0.20 & - \\
\hline 0 & 85 & $205837 \mathrm{c} 7$ & 18:10:15.10 & $-31: 54: 11.20$ & 15.89 & 1.701 & 1.95 & 1.6 & 4750 & -0.36 & 0.13 & - \\
\hline 0 & 87 & $75097 \mathrm{c} 7$ & 18:10:12.85 & $-31: 50: 30.60$ & 15.87 & 1.722 & 1.82 & 1.5 & 4550 & -0.09 & 0.17 & - \\
\hline 0 & 88 & $63747 c 7$ & $18: 10: 10.96$ & $-31: 52: 10.40$ & 15.77 & 1.560 & 2.02 & 1.5 & 4950 & -0.09 & 0.16 & - \\
\hline 0 & 90 & $46642 c 7$ & 18:10:01.82 & $-31: 54: 39.00$ & 15.94 & 1.599 & 1.91 & 1.5 & 4650 & 0.21 & 0.24 & - \\
\hline 0 & 91 & $57883 \mathrm{c} 7$ & 18:10:05.62 & $-31: 53: 01.40$ & 15.84 & 1.815 & 1.81 & 1.5 & 4550 & -0.25 & 0.16 & - \\
\hline 0 & 92 & $51688 \mathrm{c} 6$ & 18:10:17.72 & $-31: 53: 50.10$ & 15.96 & 1.721 & 1.95 & 1.5 & 4700 & -0.42 & 0.15 & - \\
\hline 0 & 93 & $209695 \mathrm{c} 7$ & 18:10:14.80 & $-31: 49: 31.70$ & 15.97 & 1.556 & 2.07 & 1.4 & 4900 & -0.11 & 0.12 & - \\
\hline 0 & 95 & $90337 \mathrm{c} 7$ & 18:10:11.51 & $-31: 48: 19.20$ & 15.91 & 1.824 & 2.02 & 1.5 & 4850 & -0.27 & 0.20 & - \\
\hline 0 & 99 & $7232 c 7$ & 18:10:04.39 & $-31: 48: 45.80$ & 15.81 & 1.476 & 2.03 & 1.5 & 4950 & -0.21 & 0.12 & - \\
\hline 0 & 100 & $480 \mathrm{c} 7$ & 18:09:46.34 & $-31: 53: 30.30$ & 15.74 & 1.473 & 2.08 & 1.5 & 5100 & -0.40 & 0.12 & - \\
\hline 0 & 102 & $860 \mathrm{c} 7$ & & $: 00.40$ & 15.88 & 1.715 & 1.80 & 1.5 & 4500 & 0.09 & 0.20 & - \\
\hline 0 & 103 & c7 & 18:10 & $-31:$ & 15.62 & 1.474 & 2.08 & 1.5 & 5200 & -0.40 & 0.13 & - \\
\hline 0 & 104 & $50439 \mathrm{c} 7$ & 18:09 & $-31: 5$ & 15.93 & 1.508 & 2.16 & 1.4 & 5100 & -0.08 & 0.18 & - \\
\hline 0 & 105 & $80144 c 7$ & 18:10:07.44 & $-31: 49: 47.10$ & 15.58 & 1.556 & 1.83 & 1.5 & 4750 & -0.05 & 0.13 & - \\
\hline 0 & 107 & $97618 \mathrm{c} 7$ & 18:09:39.57 & $-31: 47: 15.80$ & 15.51 & 1.512 & 1.88 & 1.5 & 4900 & -0.91 & 0.17 & - \\
\hline 0 & 108 & $102010 c 7$ & 18:09:54.38 & $-31: 46: 37.60$ & 15.77 & 1.606 & 1.90 & 1.4 & 4750 & -0.56 & 0.12 & - \\
\hline 0 & 109 & $7242 c 8$ & 18:09:26.69 & $-31: 48: 58.50$ & 15.92 & 1.899 & 1.67 & 1.5 & 4300 & 0.00 & 0.2 & - \\
\hline 0 & 111 & $768 \mathrm{c} 7$ & 18:09:43.40 & $-31: 48: 32.30$ & 15.86 & 1.711 & 1.72 & 1.5 & 4400 & -0.25 & 0.14 & - \\
\hline 0 & 112 & $86105 c 7$ & 18:09:44.61 & $-31: 48: 55.00$ & 15.94 & 1.621 & 2.06 & 1.4 & 4900 & -0.14 & 0.14 & - \\
\hline 0 & 113 & $77209 \mathrm{c} 7$ & 18:09:36.36 & $-31: 50: 12.00$ & 15.90 & 1.536 & 1.98 & 1.3 & 4800 & -0.43 & 0.14 & - \\
\hline 0 & 114 & $98458 \mathrm{c} 7$ & 18:10:01.97 & $-31: 47: 08.40$ & 15.93 & 1.846 & 1.68 & 1.5 & 4300 & -0.02 & 0.16 & - \\
\hline 1 & 116 & $5685 \mathrm{c} 3$ & 18:09:52.41 & $-31: 44: 13.30$ & 15.78 & 1.554 & 1.98 & 1.5 & 4900 & -1.15 & 0.16 & - \\
\hline 2 & 117 & $104943 \mathrm{c} 6$ & 18:10:16.42 & $-31: 46: 00.50$ & 15.60 & 1.442 & 2.10 & 1.5 & 5300 & -1.20 & 0.16 & NGC 6558 \\
\hline 0 & 118 & $5118 \mathrm{c} 4$ & 18:09:25.84 & $-31: 44: 15.60$ & 15.89 & 1.588 & 1.95 & 1.6 & 4750 & 0.06 & 0.17 & - \\
\hline 0 & 119 & $110465 \mathrm{c} 7$ & & & & & 1.90 & 1.4 & 4600 & 0.00 & 0.21 & - \\
\hline 0 & 120 & $212654 \mathrm{c} 8$ & 18:09:33.44 & $-31: 45: 25.00$ & 15.89 & & 2.01 & 1.4 & 48 & 0.39 & 0.26 & - \\
\hline 0 & 121 & 108191c7 & 18:09:45.55 & $-31: 45: 44.60$ & 15.90 & 1.454 & 2.11 & 1.5 & 5050 & -0.77 & 0.11 & - \\
\hline 0 & 122 & $23017 \mathrm{c} 3$ & 18:10:04.46 & $-31: 41: 45.30$ & 15.96 & 1.941 & 1.65 & 1.5 & 4250 & 0.09 & 0.21 & - \\
\hline 0 & 123 & $101167 \mathrm{c} 8$ & 18:09:20.54 & $-31: 47: 00.20$ & 15.92 & 1.496 & 2.16 & 1.5 & 5100 & 0.05 & 0.13 & - \\
\hline 0 & 124 & $103592 \mathrm{c} 7$ & 18:09:50.04 & $-31: 46: 23.70$ & 15.85 & 1.624 & 1.99 & 1.5 & 4850 & -0.31 & 0.18 & - \\
\hline 0 & 126 & $202633 \mathrm{c} 3$ & 18:10:13.06 & $-31: 41: 28.50$ & 15.71 & & 1.73 & 1.5 & 4500 & 0.31 & 0.26 & - \\
\hline 0 & 127 & $32080 \mathrm{c} 3$ & 18:09:51.13 & $-31: 40: 28.00$ & 15.63 & 1.549 & 1.99 & 1.6 & 5000 & -0.17 & 0.13 & - \\
\hline 0 & 128 & $43791 c 3$ & 18:09:37.90 & $-31: 38: 46.50$ & 15.71 & 1.534 & 2.20 & 1.3 & 4950 & 0.00 & 0.15 & - \\
\hline 0 & 129 & $204664 c 4$ & 18:09:34.75 & $-31: 38: 50.90$ & 15.72 & 1.478 & 2.22 & 1.5 & 5400 & 0.18 & 0.12 & - \\
\hline 1 & 132 & $11653 \mathrm{c} 3$ & 18:09:56.84 & $-31: 43: 22.50$ & 15.65 & 1.613 & 1.91 & 1.5 & 4850 & 0.13 & 0.22 & - \\
\hline
\end{tabular}


Table 3. continued.

\begin{tabular}{|c|c|c|c|c|c|c|c|c|c|c|c|c|}
\hline $\mathrm{QF}^{a}$ & ID & OGLE-ID & RA & Dec & $V$ & $V-I$ & $\log g$ & $V_{\mathrm{t}}$ & $T_{\text {eff }}$ & {$[\mathrm{Fe} / \mathrm{H}]$} & $\sigma^{b}$ & Cluster? \\
\hline 0 & 134 & $21259 \mathrm{c} 2$ & $18: 10: 17.72$ & $-31: 41: 55.20$ & 15.71 & 1.545 & 2.02 & 1.5 & 5000 & -0.08 & 0.14 & - \\
\hline 0 & 3 & $200810 \mathrm{c} 3$ & $18: 10: 13.35$ & $-31: 43: 37.70$ & 16.28 & 2.117 & 1.66 & 1.5 & 4100 & 0.11 & 0.19 & - \\
\hline 0 & 5 & $34058 \mathrm{c} 3$ & 18:10:04.37 & $-31: 40: 10.90$ & 16.16 & 1.686 & 1.84 & 1.6 & 4400 & 0.21 & 0.19 & - \\
\hline 0 & 6 & $47752 \mathrm{c} 3$ & 18:09:59.53 & $-31: 38: 14.10$ & 16.31 & 1.940 & 1.47 & 1.5 & 3900 & 0.20 & 0.20 & - \\
\hline 0 & 8 & $40528 \mathrm{c} 3$ & 18:09:58.10 & $-31: 39: 15.20$ & 16.17 & 1.850 & 1.88 & 1.7 & 4450 & -0.48 & 0.14 & - \\
\hline 0 & 10 & $29280 \mathrm{c} 3$ & 18:09:50.48 & $-31: 40: 51.60$ & 16.15 & 1.855 & 1.80 & 1.5 & 4350 & 0.18 & 0.20 & - \\
\hline 2 & 11 & $12982 \mathrm{c} 3$ & 18:10:07.98 & $-31: 43: 11.20$ & 16.61 & 2.426 & 1.95 & 1.5 & 4300 & 0.01 & 0.50 & - \\
\hline 0 & 13 & $108051 \mathrm{c} 7$ & 18:09:55.95 & $-31: 45: 46.30$ & 16.29 & 2.107 & 1.79 & 1.6 & 4250 & -0.09 & 0.21 & - \\
\hline 0 & 15 & $20863 \mathrm{c} 2$ & 18:10:22.73 & $-31: 41: 58.80$ & 16.30 & 2.006 & 1.75 & 1.5 & 4200 & 0.08 & 0.23 & - \\
\hline 0 & 16 & $31220 \mathrm{c} 2$ & 18:10:19.06 & $-31: 40: 28.10$ & 16.09 & 1.615 & 1.81 & 1.7 & 4400 & -0.02 & 0.17 & - \\
\hline 1 & 17 & $50086 \mathrm{c} 2$ & $18: 10: 15.56$ & $-31: 37: 40.00$ & 16.15 & 1.804 & 1.80 & 1.5 & 4350 & 0.32 & 0.26 & - \\
\hline 0 & 18 & $208608 \mathrm{c} 3$ & 18:10:14.06 & $-31: 33: 52.00$ & 16.17 & 1.751 & 2.06 & 1.5 & 4750 & -0.56 & 0.13 & - \\
\hline 0 & 20 & $58159 \mathrm{c} 3$ & 18:10:08.82 & $-31: 36: 44.50$ & 16.38 & 1.895 & 1.93 & 1.5 & 4400 & -0.09 & 0.15 & - \\
\hline 2 & 21 & $70770 \mathrm{c} 3$ & 18:10:06.95 & $-31: 34: 58.40$ & 16.27 & 1.949 & 1.61 & 1.5 & 4050 & -0.04 & 0.31 & - \\
\hline 0 & 23 & $205096 \mathrm{c} 2$ & 18:10:39.82 & $-31: 38: 06.90$ & 16.18 & 1.792 & 2.10 & 1.4 & 4800 & -0.15 & 0.12 & - \\
\hline 0 & 24 & $148090 \mathrm{c} 2$ & 18:10:32.27 & $-31: 39: 15.10$ & 16.20 & 1.767 & 2.05 & 1.5 & 4700 & -0.28 & 0.16 & - \\
\hline 0 & 25 & $42348 \mathrm{c} 2$ & 18:10:32.46 & $-31: 38: 48.90$ & 16.16 & 1.785 & 1.84 & 1.5 & 4400 & -0.09 & 0.15 & - \\
\hline 0 & 27 & $149531 \mathrm{c} 2$ & 18:10:27.61 & $-31: 38: 49.70$ & 16.15 & 1.810 & 1.91 & 1.5 & 4500 & -0.38 & 0.12 & - \\
\hline 0 & 28 & $31090 \mathrm{c} 2$ & 18:10:37.38 & $-31: 40: 29.10$ & 16.04 & 1.671 & 1.98 & 1.5 & 4700 & -0.26 & 0.11 & - \\
\hline 1 & 29 & $14261 \mathrm{c} 2$ & 18:10:16.93 & $-31: 42: 57.50$ & 16.23 & 1.838 & 1.72 & 1.4 & 4200 & 0.40 & 0.24 & - \\
\hline 0 & 30 & 69986c2 & 18:10:28.56 & $-31: 34: 46.20$ & 16.19 & 1.781 & 2.01 & 1.5 & 4650 & -0.27 & 0.14 & - \\
\hline 1 & 31 & $73344 c 2$ & 18:10:27.05 & $-31: 34: 15.80$ & 16.52 & 2.252 & 2.05 & 1.5 & 4500 & -0.00 & 0.30 & - \\
\hline 1 & 34 & $139560 \mathrm{c} 2$ & 18:10:45.15 & $-31: 41: 57.90$ & 16.42 & 2.040 & 1.87 & 1.5 & 4300 & 0.26 & 0.33 & - \\
\hline 2 & 35 & $145595 \mathrm{c} 2$ & $18: 10: 39.53$ & $-31: 39: 58.90$ & 16.27 & 2.169 & 1.81 & 1.5 & 4300 & 0.44 & 0.39 & - \\
\hline 0 & 36 & $22905 \mathrm{c} 2$ & 18:10:28.24 & $-31: 41: 40.70$ & 16.10 & 1.673 & 1.89 & 1.4 & 4500 & -0.45 & 0.12 & - \\
\hline 0 & 37 & $47298 \mathrm{c} 2$ & 18:10:49.67 & $-31: 38: 04.10$ & 16.10 & 1.853 & 1.79 & 1.7 & 4350 & -0.51 & 0.12 & - \\
\hline 1 & 38 & $33601 \mathrm{c} 1$ & 18:10: & $-31: 39: 56.60$ & 16.42 & 2.059 & 1.94 & 1.6 & 4400 & 0.29 & 0.27 & - \\
\hline 0 & 39 & $43023 c 2$ & 18:10:38.81 & $-31: 38: 42.60$ & 16.10 & 1.718 & 1.95 & 1.4 & 4600 & -0.46 & 0.16 & - \\
\hline 2 & 42 & $107527 \mathrm{c} 6$ & 18:10:17.65 & $-31: 45: 38.90$ & 16.05 & 1.612 & 1.20 & 1.5 & 3750 & -0.96 & 0.18 & NGC 6558 \\
\hline 0 & 43 & $31176 \mathrm{c} 2$ & $18: 10: 44.56$ & $-31: 40: 28.40$ & 16.01 & 1.875 & 1.76 & 1.5 & 4400 & -0.22 & 0.13 & - \\
\hline 0 & 44 & $17038 \mathrm{c} 2$ & $18: 10: 36.15$ & $-31: 42: 32.70$ & 16.14 & 1.748 & 1.76 & 1.4 & 4300 & 0.29 & 0.22 & - \\
\hline 1 & 45 & $103742 \mathrm{c} 5$ & 18:10:58.15 & $-31: 45: 48.00$ & 16.37 & 1.929 & 1.69 & 1.5 & 4100 & 0.31 & 0.19 & - \\
\hline 0 & 49 & $959 \mathrm{c} 2$ & 18:10:33.83 & $-31: 44: 56.10$ & 16.16 & 1.686 & 2.00 & 1.4 & 4650 & -0.58 & 0.12 & - \\
\hline 0 & 51 & $10584 \mathrm{c} 2$ & $18: 10: 47.85$ & $-31: 43: 29.80$ & 16.17 & 1.751 & 1.91 & 1.4 & 4500 & -0.38 & 0.13 & - \\
\hline 0 & 52 & $15094 \mathrm{c} 1$ & 18:10:55.31 & $-31: 42: 44.40$ & 16.25 & 1.797 & 2.01 & 1.4 & 4600 & -0.19 & 0.15 & - \\
\hline 0 & 54 & 95371c6 & 18:10:34.70 & $-31: 47: 23.70$ & 16.20 & 1.817 & 1.92 & 1.4 & 4500 & -0.27 & 0.15 & - \\
\hline 0 & 55 & $98734 \mathrm{c} 6$ & 18:10: & $-31: 4$ & 16.06 & 1.654 & 1.99 & 1.1 & 4700 & -0.06 & 0.17 & - \\
\hline 0 & 56 & $103413 \mathrm{c} 6$ & 18:10:52.64 & $-31: 46: 13.00$ & 16.16 & 1.798 & 1.65 & 1.4 & 4150 & 0.24 & 0.25 & - \\
\hline 0 & 57 & $5 \mathrm{c} 5$ & 18:10 & $-31: 2$ & 16.39 & 2.224 & 1.82 & 1.5 & 4250 & 0.15 & 0.23 & - \\
\hline 0 & 58 & $91631 c 6$ & 18:10: & $-31: 47: 56.80$ & 16.07 & 1.608 & 2.05 & 1.5 & 4800 & -0.28 & 0.15 & - \\
\hline 0 & 59 & $95545 \mathrm{c} 6$ & $18: 10: 49.00$ & $-31: 47: 22.00$ & 16.25 & 1.892 & 1.77 & 1.3 & 4250 & -0.45 & 0.12 & - \\
\hline 1 & 61 & $96460 c 6$ & 18:10:50.03 & $-31: 47: 14.00$ & 16.01 & 1.685 & 1.94 & 1.4 & 4650 & 0.25 & 0.21 & - \\
\hline 1 & 62 & $83500 c 6$ & 18:10:33.98 & $-31: 49: 09.10$ & 16.40 & 2.172 & 2.00 & 1.4 & 4400 & 0.09 & 0.30 & - \\
\hline 0 & 64 & $72513 c 6$ & $18: 10: 43.80$ & $-31: 50: 46.30$ & 16.04 & 1.624 & 1.98 & 1.7 & 4700 & -0.48 & 0.16 & - \\
\hline 0 & 65 & 69731c6 & 18:10:35.15 & $-31: 51: 10.60$ & 16.34 & 1.913 & 1.95 & 1.5 & 4450 & -0.19 & 0.19 & - \\
\hline 1 & 66 & $73072 c 6$ & 18:10:49.86 & $-31: 50: 41.50$ & 16.25 & 2.132 & 1.60 & 1.5 & 4050 & 0.12 & 0.28 & - \\
\hline 0 & 68 & $56641 \mathrm{c} 6$ & 18:10:38.77 & $-31: 53: 06.90$ & 16.00 & 1.675 & 1.81 & 1.5 & 4450 & 0.17 & 0.20 & - \\
\hline 0 & 69 & $208907 \mathrm{c} 6$ & 18:10:53.38 & $-31: 50: 12.50$ & 16.03 & 1.581 & 2.09 & 1.5 & 4900 & -0.17 & 0.15 & - \\
\hline 0 & 70 & $99166 \mathrm{c6}$ & 18:10:23.01 & $-31: 46: 49.60$ & 16.26 & 1.894 & 1.81 & 1.4 & 4300 & 0.04 & 0.23 & - \\
\hline 0 & 71 & $71832 \mathrm{c} 6$ & 18:10:29.83 & $-31: 50: 52.30$ & 16.24 & 1.963 & 1.80 & 1.4 & 4300 & -0.09 & 0.19 & - \\
\hline 0 & 72 & 77481c6 & 18:10:43.18 & $-31: 50: 02.10$ & 16.05 & 1.555 & 2.13 & 1.5 & 4950 & -0.28 & 0.09 & - \\
\hline 0 & 73 & $91776 \mathrm{c} 6$ & 18:10:32.31 & $-31: 47: 55.50$ & 16.20 & 1.751 & 2.11 & 1.5 & 4800 & 0.23 & 0.24 & - \\
\hline 0 & 74 & $93621 \mathrm{c} 6$ & 18:10:24.21 & $-31: 47: 39.30$ & 16.11 & 1.727 & 1.92 & 1.5 & 4550 & -0.22 & 0.17 & - \\
\hline 0 & 75 & 99940c6 & 18:10:15.34 & $-31: 46: 43.10$ & 16.13 & 1.771 & 1.69 & 1.6 & 4200 & -0.25 & 0.12 & - \\
\hline 0 & 78 & $53554 \mathrm{c} 6$ & 18:10:20.77 & $-31: 53: 33.40$ & 16.10 & 1.910 & 1.78 & 1.4 & 4350 & 0.08 & 0.21 & - \\
\hline 0 & 79 & $63690 \mathrm{c} 6$ & 18:10:22.57 & $-31: 52: 04.20$ & 16.08 & 1.607 & 2.06 & 1.5 & 4800 & 0.03 & 0.16 & - \\
\hline 0 & 80 & 205097c6 & 18:10:26.99 & $-31: 54: 51.70$ & 16.54 & 2.279 & 2.18 & 1.4 & 4700 & 0.26 & 0.21 & - \\
\hline 0 & 81 & $52922 \mathrm{c} 6$ & 18:10:27.94 & $-31: 53: 39.10$ & 16.24 & 1.753 & 1.94 & 1.5 & 4500 & -0.30 & 0.14 & - \\
\hline 0 & 82 & $51954 c 6$ & 18:10:29.15 & $-31: 53: 47.60$ & 16.14 & 1.756 & 1.76 & 1.5 & 4300 & 0.03 & 0.22 & - \\
\hline 0 & 83 & $56533 c 6$ & 18:10:32.58 & $-31: 53: 07.70$ & 16.05 & 1.670 & 1.72 & 1.5 & 4300 & 0.13 & 0.30 & - \\
\hline 0 & 84 & $94909 \mathrm{c} 7$ & 18:10:12.67 & $-31: 47: 39.80$ & 16.14 & 2.112 & 1.73 & 1.4 & 4250 & -0.11 & 0.26 & - \\
\hline 0 & 85 & $73484 \mathrm{c} 7$ & 18:10:12.00 & $-31: 50: 44.70$ & 16.40 & 1.988 & 2.07 & 1.2 & 4600 & 0.31 & 0.25 & - \\
\hline 0 & 86 & $90995 \mathrm{c} 7$ & 18:10:09.65 & $-31: 48: 13.70$ & 16.11 & 1.619 & 2.07 & 1.4 & 4800 & -0.03 & 0.18 & - \\
\hline
\end{tabular}


Table 3. continued.

\begin{tabular}{|c|c|c|c|c|c|c|c|c|c|c|c|c|}
\hline $\mathrm{QF}^{a}$ & ID & OGLE-ID & RA & Dec & $V$ & $V-I$ & $\log g$ & $V_{\mathrm{t}}$ & $T_{\text {eff }}$ & {$[\mathrm{Fe} / \mathrm{H}]$} & $\sigma^{b}$ & Cluster? \\
\hline 0 & 87 & $41505 \mathrm{c} 7$ & $18: 10: 07.59$ & $-31: 55: 23.30$ & 16.09 & 1.670 & 2.06 & 1.6 & 4800 & -0.48 & 0.15 & - \\
\hline 1 & 88 & $34034 c 7$ & 18:10:03.84 & $-31: 56: 28.90$ & 16.33 & 2.064 & 1.87 & 1.5 & 4350 & 0.04 & 0.24 & - \\
\hline 1 & 89 & $205852 \mathrm{c} 7$ & $18: 10: 13.63$ & $-31: 54: 09.60$ & 16.30 & 1.797 & 1.86 & 1.5 & 4350 & 0.06 & 0.31 & - \\
\hline 1 & 90 & $64944 \mathrm{c} 7$ & 18:10:02.47 & $-31: 51: 59.80$ & 16.20 & 2.088 & 1.79 & 1.5 & 4350 & 0.08 & 0.23 & - \\
\hline 0 & 91 & $75601 \mathrm{c} 7$ & 18:10:09.16 & $-31: 50: 26.30$ & 16.03 & 1.891 & 1.79 & 1.5 & 4400 & -0.08 & 0.28 & - \\
\hline 0 & 92 & $60208 \mathrm{c} 7$ & 18:10:07.77 & $-31: 52: 41.30$ & 16.12 & 1.722 & 1.83 & 1.6 & 4400 & -0.37 & 0.09 & - \\
\hline 0 & 93 & $46088 \mathrm{c} 7$ & 18:10:08.96 & $-31: 54: 43.70$ & 16.14 & 1.869 & 1.90 & 1.4 & 4500 & -0.20 & 0.16 & - \\
\hline 1 & 95 & $77743 \mathrm{c} 7$ & 18:09:49.10 & $-31: 50: 07.60$ & 16.09 & 1.703 & 1.78 & 1.5 & 4350 & 0.25 & 0.22 & - \\
\hline 0 & 96 & $85832 \mathrm{c} 7$ & 18:09:52.99 & $-31: 48: 57.70$ & 16.25 & 1.884 & 1.77 & 1.5 & 4250 & 0.01 & 0.20 & - \\
\hline 2 & 97 & $211927 \mathrm{c} 7$ & 18:10:15.21 & $-31: 46: 00.60$ & 16.04 & 1.620 & 2.06 & 1.0 & 4850 & -0.81 & 0.24 & NGC 6558 \\
\hline 1 & 98 & 93881c7 & 18:10:02.82 & $-31: 47: 48.50$ & 16.13 & 1.882 & 1.83 & 1.5 & 4400 & 0.03 & 0.25 & - \\
\hline 0 & 99 & $82739 c 7$ & 18:09:58.42 & $-31: 49: 24.60$ & 16.20 & 1.781 & 1.99 & 1.5 & 4600 & -0.45 & 0.14 & - \\
\hline 0 & 100 & $88860 \mathrm{c} 7$ & 18:10:03.71 & $-31: 48: 31.80$ & 16.09 & 1.884 & 1.85 & 1.5 & 4450 & -0.06 & 0.20 & - \\
\hline 0 & 101 & $62874 c 7$ & 18:09:51.36 & $-31: 52: 17.70$ & 16.03 & 1.820 & 1.92 & 1.5 & 4600 & -0.48 & 0.17 & - \\
\hline 0 & 102 & $73636 c 7$ & 18:09:56.93 & $-31: 50: 43.20$ & 16.11 & 1.686 & 1.86 & 1.5 & 4450 & -0.22 & 0.12 & - \\
\hline 0 & 103 & $56730 c 7$ & 18:09:53.17 & $-31: 53: 10.80$ & 16.31 & 1.980 & 1.83 & 1.3 & 4300 & 0.00 & 0.21 & - \\
\hline 0 & 104 & $48678 \mathrm{c} 7$ & 18:09:52.26 & $-31: 54: 21.10$ & 16.02 & 1.622 & 2.00 & 1.6 & 4750 & 0.17 & 0.24 & - \\
\hline 0 & 105 & $58592 \mathrm{c} 7$ & 18:09:57.44 & $-31: 52: 55.10$ & 16.20 & 1.908 & 1.95 & 1.6 & 4550 & 0.17 & 0.22 & - \\
\hline 0 & 106 & $77419 c 7$ & 18:10:04.15 & $-31: 50: 11.00$ & 16.05 & 1.615 & 1.93 & 1.5 & 4600 & 0.18 & 0.21 & - \\
\hline 1 & 107 & $96001 \mathrm{c} 8$ & 18:09:28.48 & $-31: 47: 44.30$ & 16.05 & 1.603 & 1.89 & 1.5 & 4550 & 0.21 & 0.26 & - \\
\hline 0 & 108 & $97453 \mathrm{c} 7$ & 18:09:39.52 & $-31: 47: 17.50$ & 16.07 & 1.580 & 2.00 & 1.5 & 4700 & 0.02 & 0.17 & - \\
\hline 0 & 109 & $105594 \mathrm{c} 7$ & 18:10:01.14 & $-31: 46: 06.90$ & 16.06 & 1.591 & 2.10 & 1.6 & 4900 & -0.25 & 0.11 & - \\
\hline 1 & 110 & $80262 \mathrm{c} 8$ & 18:09:20.06 & $-31: 49: 58.20$ & 16.22 & 1.766 & 2.06 & 1.5 & 4700 & 0.23 & 0.23 & - \\
\hline 0 & 111 & $80419 c 8$ & 18:09:26.94 & 9:57.00 & 16.08 & 1.767 & 1.81 & 1.5 & 4400 & 0.18 & 0.24 & - \\
\hline 1 & 112 & $98090 \mathrm{c} 7$ & 18:09:53.69 & $-31: 47: 11.80$ & 16.01 & 1.766 & 1.88 & 1.6 & 4550 & -0.05 & 0.34 & - \\
\hline 0 & 113 & $75382 c 8$ & 18:09:31.58 & $-31: 50: 39.60$ & 16.18 & 1.748 & 1.88 & 1.5 & 4450 & 0.20 & 0.24 & - \\
\hline 0 & 114 & $94445 c 7$ & 18:09:57.05 & $-31: 47: 43.40$ & 16.14 & 1.740 & 1.93 & 1.5 & 4550 & -0.29 & 0.12 & - \\
\hline 0 & 115 & $97069 \mathrm{c} 7$ & 18:10:02.43 & $-31: 47: 21.10$ & 16.16 & 2.120 & 1.61 & 1.5 & 4100 & 0.25 & 0.48 & - \\
\hline 2 & 116 & $132843 \mathrm{c} 3$ & 18:09:46.60 & $-31: 44: 16.00$ & 16.15 & 2.019 & 2.00 & 1.5 & 4650 & -0.05 & 0.55 & - \\
\hline 0 & 117 & $8683 \mathrm{c} 4$ & 18:09:19.59 & $-31: 43: 43.60$ & 16.05 & 1.907 & 1.69 & 1.4 & 4250 & 0.02 & 0.18 & - \\
\hline 0 & 118 & $215027 \mathrm{c} 7$ & 18:10:05.98 & $-31: 44: 49.20$ & 16.35 & 1.872 & 1.68 & 1.5 & 4100 & 0.20 & 0.24 & - \\
\hline 0 & 119 & $213817 \mathrm{c} 7$ & 18:10:03.06 & $-31: 45: 00.90$ & 16.13 & 1.687 & 1.86 & 1.5 & 4450 & 0.27 & 0.26 & - \\
\hline 0 & 120 & $110776 \mathrm{c} 7$ & 18:09:45.13 & $-31: 45: 22.40$ & 15.82 & 1.610 & 1.84 & 1.5 & 4600 & -0.85 & 0.15 & - \\
\hline 0 & 121 & $111007 \mathrm{c} 8$ & 18:09:27.43 & $-31: 45: 37.90$ & 16.26 & 1.835 & 1.81 & 1.4 & 4300 & 0.01 & 0.20 & - \\
\hline 0 & 123 & $108627 \mathrm{c} 7$ & 18:10:04.95 & $-31: 45: 40.80$ & 16.31 & 2.043 & 2.03 & 1.4 & 4600 & 0.02 & 0.29 & - \\
\hline 0 & 124 & $7461 \mathrm{c} 8$ & 18:09:21.33 & $-31: 47: 31.60$ & 16.01 & 1.702 & 1.97 & 1.5 & 4700 & 0.31 & 0.26 & - \\
\hline 2 & 126 & $9 \mathrm{c} 3$ & $18: 0$ & -31 & 16.59 & 2.230 & 2.01 & 1.5 & 4400 & 0.30 & 0.30 & - \\
\hline 0 & 128 & $346 \mathrm{c} 3$ & 18:09:55.66 & $2: 16.50$ & 16.02 & 1.560 & 2.09 & 1.5 & 4900 & 0.27 & 0.15 & - \\
\hline 0 & 129 & 4111 & 18:0 & $-31:$ & 16.10 & 1.719 & 1.89 & 1.5 & 4500 & -0.31 & 0.13 & - \\
\hline 0 & 130 & $9081 c 3$ & 18:10:01.92 & $-31: 43: 44.80$ & 16.22 & 1.782 & 1.93 & 1.5 & 4500 & -0.25 & 0.15 & - \\
\hline 0 & 131 & $35643 \mathrm{c} 4$ & 18:09:29.06 & $-31: 39: 54.20$ & 16.01 & 1.522 & 2.17 & 1.6 & 5050 & 0.22 & 0.17 & - \\
\hline 0 & 134 & $27350 \mathrm{c} 4$ & 18:09:23.23 & $-31: 41: 03.90$ & 16.07 & 1.595 & 2.08 & 1.3 & 4850 & -0.36 & 0.19 & - \\
\hline 0 & 135 & $6693 c 3$ & 18:09:55.37 & $-31: 44: 04.70$ & 16.17 & 1.675 & 2.01 & 1.5 & 4650 & -0.03 & 0.16 & - \\
\hline \multicolumn{13}{|c|}{ Field at $b=-12^{\circ}$} \\
\hline 0 & 3 & $2002 C 3$ & $18: 35: 06.73$ & $-34: 31: 59.59$ & 15.57 & 1.250 & 2.31 & 1.2 & 5000 & -0.36 & 0.17 & - \\
\hline 0 & 4 & $2374 \mathrm{C} 3$ & 18:35:08.04 & $-34: 31: 13.37$ & 15.76 & 1.244 & 2.38 & 1.6 & 5000 & -0.58 & 0.15 & - \\
\hline 0 & 5 & $3142 \mathrm{C} 3$ & 18:35:09.48 & $-34: 29: 28.15$ & 15.95 & 1.287 & 2.41 & 1.4 & 4900 & -0.15 & 0.16 & - \\
\hline 0 & 6 & 2947C3 & 18:35:00.59 & $-34: 29: 55.64$ & 16.02 & 1.204 & 2.51 & 1.2 & 5050 & -0.44 & 0.16 & - \\
\hline 0 & 7 & $2200 \mathrm{C} 3$ & 18:34:57.77 & $-34: 31: 35.91$ & 16.15 & 1.315 & 2.43 & 1.5 & 4800 & -0.17 & 0.14 & - \\
\hline 0 & 9 & 3018C3 & 18:35:03.59 & $-34: 29: 45.23$ & 15.49 & 1.404 & 2.08 & 1.4 & 4650 & -0.61 & 0.12 & - \\
\hline 0 & 10 & $3515 \mathrm{C} 5$ & 18:34:50.72 & $-34: 28: 37.94$ & 15.18 & 1.355 & 2.04 & 1.6 & 4800 & 0.00 & 0.16 & - \\
\hline 0 & 11 & 2769C3 & 18:34:57.52 & $-34: 30: 21.29$ & 15.78 & 1.295 & 2.45 & 1.5 & 5100 & 0.18 & 0.17 & - \\
\hline 2 & 12 & 3109C3 & 18:34:45.67 & $-34: 29: 31.70$ & 15.81 & 1.341 & 2.35 & 1.3 & 4900 & -0.58 & 0.26 & - \\
\hline 2 & 13 & $201583 \mathrm{C} 3$ & 18:34:51.39 & $-34: 31: 40.37$ & 15.79 & 1.258 & 2.36 & 1.4 & 4950 & -1.18 & 0.18 & - \\
\hline 0 & 15 & $2470 \mathrm{C} 3$ & $18: 35: 21.52$ & $-34: 31: 01.85$ & 16.25 & 1.375 & 2.47 & 1.5 & 4800 & -0.06 & 0.16 & - \\
\hline 0 & 16 & $3267 \mathrm{C} 3$ & $18: 35: 22.06$ & $-34: 29: 11.16$ & 15.07 & 1.349 & 2.03 & 1.2 & 4850 & 0.01 & 0.14 & - \\
\hline 0 & 17 & $3161 \mathrm{C} 3$ & $18: 35: 19.42$ & $-34: 29: 26.65$ & 15.46 & 1.306 & 2.27 & 1.6 & 5000 & -0.12 & 0.12 & - \\
\hline 0 & 19 & $4365 \mathrm{C} 3$ & 18:35:21.17 & $-34: 26: 50.97$ & 16.08 & 1.148 & 2.53 & 1.6 & 5050 & -0.66 & 0.15 & - \\
\hline 0 & 20 & 6549C6 & 18:35:03.43 & $-34: 37: 24.28$ & 15.70 & 1.320 & 2.31 & 1.3 & 4900 & 0.11 & 0.15 & - \\
\hline 0 & 21 & $3733 \mathrm{C} 3$ & 18:35:17.99 & $-34: 28: 09.31$ & 15.10 & 1.423 & 1.98 & 1.4 & 4750 & -0.19 & 0.12 & - \\
\hline 0 & 22 & $4085 \mathrm{C} 3$ & 18:35:13.57 & $-34: 27: 23.97$ & 16.13 & 1.513 & 2.31 & 1.3 & 4600 & -0.38 & 0.13 & - \\
\hline 0 & 24 & $2525 \mathrm{C} 2$ & $18: 35: 46.60$ & $-34: 30: 20.41$ & 15.99 & 1.214 & 2.48 & 1.4 & 5000 & -0.33 & 0.12 & - \\
\hline 0 & 28 & $6505 \mathrm{C} 6$ & 18:35:18.68 & $-34: 37: 30.83$ & 16.04 & 1.232 & 2.50 & 1.4 & 5000 & -0.37 & 0.14 & - \\
\hline
\end{tabular}


Table 3. continued.

\begin{tabular}{|c|c|c|c|c|c|c|c|c|c|c|c|c|}
\hline $\mathrm{QF}^{a}$ & ID & OGLE-ID & RA & Dec & $V$ & $V-I$ & $\log g$ & $V_{\mathrm{t}}$ & $T_{\text {eff }}$ & {$[\mathrm{Fe} / \mathrm{H}]$} & $\sigma^{b}$ & Cluster? \\
\hline 0 & 29 & $867 \mathrm{C} 3$ & 18:35:09.10 & $-34: 34: 34.73$ & 15.76 & 1.228 & 2.38 & 1.4 & 5000 & -0.46 & 0.12 & - \\
\hline 0 & 31 & $222 \mathrm{C} 3$ & $18: 35: 14.90$ & $-34: 36: 00.45$ & 15.50 & 1.295 & 2.23 & 1.6 & 4900 & -0.38 & 0.12 & _- \\
\hline 0 & 33 & $650 \mathrm{C} 2$ & $18: 35: 56.80$ & $-34: 34: 48.25$ & 15.29 & 1.530 & 1.81 & 1.4 & 4350 & -0.67 & 0.10 & - \\
\hline 0 & 35 & $2296 C 2$ & $18: 35: 51.98$ & $-34: 30: 52.18$ & 15.90 & 1.223 & 2.54 & 1.5 & 5200 & 0.03 & 0.14 & - \\
\hline 0 & 36 & $1876 \mathrm{C} 2$ & $18: 35: 49.38$ & $-34: 31: 56.10$ & 15.86 & 1.106 & 2.54 & 1.4 & 5250 & -0.86 & 0.13 & - \\
\hline 0 & 37 & $2335 \mathrm{C} 2$ & $18: 35: 58.85$ & $-34: 30: 46.05$ & 15.81 & 1.270 & 2.26 & 1.2 & 4750 & -0.84 & 0.15 & - \\
\hline 0 & 38 & $1814 \mathrm{C} 1$ & $18: 36: 11.58$ & $-34: 32: 05.99$ & 15.85 & 1.427 & 2.22 & 1.3 & 4650 & -0.39 & 0.12 & - \\
\hline 2 & 40 & $2052 \mathrm{C} 2$ & $18: 35: 59.96$ & $-34: 31: 27.23$ & 15.42 & 1.145 & 2.27 & 1.5 & 5050 & -0.94 & 0.30 & - \\
\hline 0 & 41 & $1156 \mathrm{C} 2$ & $18: 35: 56.02$ & $-34: 33: 36.40$ & 15.93 & 1.534 & 1.95 & 1.1 & 4200 & -0.36 & 0.13 & - \\
\hline 0 & 42 & $2407 \mathrm{C} 2$ & 18:36:01.99 & $-34: 30: 36.77$ & 15.49 & 1.173 & 2.25 & 1.5 & 4950 & -0.70 & 0.12 & - \\
\hline 0 & 43 & $1918 \mathrm{C} 1$ & $18: 36: 07.53$ & $-34: 31: 51.05$ & 15.83 & 1.248 & 2.36 & 1.4 & 4900 & -0.36 & 0.15 & - \\
\hline 0 & 44 & $1917 \mathrm{C} 1$ & $18: 36: 12.50$ & $-34: 31: 51.47$ & 16.22 & 1.426 & 2.31 & 1.4 & 4550 & 0.18 & 0.29 & - \\
\hline 0 & 45 & $6080 \mathrm{C} 8$ & $18: 36: 20.59$ & $-34: 38: 24.15$ & 15.89 & 1.358 & 2.33 & 1.4 & 4800 & 0.11 & 0.26 & - \\
\hline 0 & 46 & $6426 \mathrm{C} 8$ & $18: 36: 10.10$ & $-34: 37: 32.99$ & 15.79 & 1.280 & 2.34 & 1.2 & 4900 & -0.39 & 0.15 & - \\
\hline 0 & 47 & $6391 \mathrm{C} 8$ & $18: 36: 23.58$ & $-34: 37: 37.81$ & 15.28 & 1.140 & 2.21 & 1.3 & 5050 & -0.59 & 0.13 & - \\
\hline 0 & 48 & $6637 \mathrm{C} 8$ & 18:36:07.58 & $-34: 37: 02.88$ & 16.07 & 1.296 & 2.40 & 1.5 & 4800 & -0.26 & 0.14 & - \\
\hline 0 & 50 & $431 \mathrm{C} 2$ & $18: 35: 53.60$ & $-34: 35: 21.07$ & 15.63 & 1.175 & 2.41 & 1.3 & 5150 & -0.02 & 0.15 & - \\
\hline 0 & 51 & $455 \mathrm{C} 1$ & $18: 36: 17.85$ & $-34: 35: 21.06$ & 15.67 & 1.174 & 2.32 & 1.4 & 4950 & -0.57 & 0.15 & - \\
\hline 0 & 52 & $6828 \mathrm{C} 7$ & $18: 35: 56.25$ & $-34: 36: 54.38$ & 16.09 & 1.360 & 2.26 & 1.4 & 4550 & 0.17 & 0.17 & - \\
\hline 2 & 53 & $608 \mathrm{C} 1$ & 18:36:13.69 & $-34: 34: 55.95$ & 15.13 & 1.133 & 2.16 & 1.2 & 5100 & -1.66 & 0.19 & - \\
\hline 0 & 56 & $5487 \mathrm{C} 8$ & $18: 36: 14.63$ & $-34: 39: 56.70$ & 16.16 & 1.339 & 2.49 & 1.5 & 4900 & -0.27 & 0.14 & - \\
\hline 0 & 57 & $4478 \mathrm{C} 8$ & $18: 36: 16.70$ & $-34: 42: 25.80$ & 15.79 & 1.260 & 2.34 & 1.4 & 4900 & -0.28 & 0.14 & - \\
\hline 0 & 59 & $4740 \mathrm{C} 8$ & 18:36:19.30 & $-34: 41: 47.15$ & 15.80 & 1.208 & 2.40 & 1.5 & 5000 & -0.33 & 0.16 & - \\
\hline 0 & 60 & $6913 \mathrm{C} 7$ & $18: 35: 52.67$ & $-34: 36: 43.31$ & 15.95 & 1.244 & 2.43 & 1.8 & 4950 & -0.21 & 0.15 & - \\
\hline 0 & 61 & $5351 \mathrm{C} 8$ & $18: 36: 08.00$ & $-34: 40: 16.01$ & 15.83 & 1.382 & 2.25 & 1.3 & 4700 & -0.09 & 0.11 & - \\
\hline 0 & 62 & $5400 \mathrm{C} 8$ & 18:36:12.37 & $-34: 40: 07.75$ & 15.96 & 1.113 & 2.54 & 1.4 & 5150 & 0.14 & 0.14 & - \\
\hline 0 & 64 & $2812 \mathrm{C} 8$ & $18: 36: 09.28$ & $-34: 46: 23.50$ & 15.63 & 1.458 & 1.95 & 1.2 & 4350 & -0.62 & 0.14 & - \\
\hline 0 & 65 & $3805 \mathrm{C} 7$ & $18: 35: 28.05$ & $-34: 43: 52.10$ & 15.70 & 1.342 & 2.43 & 1.4 & 5150 & -0.38 & 0.14 & - \\
\hline 0 & 68 & $2772 \mathrm{C} 7$ & $18: 35: 48.07$ & $-34: 46: 25.83$ & 15.10 & 1.258 & 2.07 & 1.4 & 4900 & -0.22 & 0.14 & - \\
\hline 0 & 70 & $3191 \mathrm{C} 7$ & $18: 35: 55.61$ & $-34: 45: 21.91$ & 15.40 & 1.243 & 2.24 & 1.4 & 5000 & -0.28 & 0.12 & - \\
\hline 1 & 71 & $2476 \mathrm{C} 7$ & $18: 35: 59.67$ & $-34: 47: 07.64$ & 15.96 & 1.120 & 2.51 & 1.4 & 5100 & -0.66 & 0.19 & - \\
\hline 0 & 72 & $3091 \mathrm{C} 8$ & 18:36:08.87 & $-34: 45: 43.81$ & 15.32 & 1.236 & 2.21 & 1.4 & 5000 & -0.36 & 0.15 & - \\
\hline 1 & 74 & $3711 \mathrm{C} 7$ & $18: 35: 41.05$ & $-34: 44: 06.11$ & 15.71 & 1.097 & 2.58 & 1.4 & 5450 & -0.30 & 0.16 & - \\
\hline 0 & 75 & $3035 \mathrm{C} 7$ & $18: 35: 51.36$ & $-34: 45: 45.52$ & 15.68 & 1.191 & 2.27 & 1.4 & 4850 & -0.61 & 0.14 & - \\
\hline 2 & 76 & $1614 \mathrm{C} 7$ & $18: 35: 28.16$ & $-34: 49: 06.49$ & 15.27 & 1.142 & 2.10 & 1.3 & 4850 & -0.73 & 0.33 & - \\
\hline 2 & 77 & $1140 \mathrm{C} 7$ & $18: 35: 31.64$ & $-34: 50: 16.35$ & 16.23 & 1.355 & 2.42 & 1.4 & 5600 & 0.60 & 0.23 & - \\
\hline 0 & 78 & $1491 \mathrm{C} 7$ & $18: 35: 33.53$ & $-34: 49: 23.93$ & 15.80 & 1.419 & 2.23 & 1.4 & 4700 & -0.19 & 0.17 & - \\
\hline 0 & 79 & $2110 \mathrm{C} 7$ & $18: 35: 36.10$ & $-34: 47: 56.62$ & 15.59 & 1.405 & 2.21 & 1.4 & 4800 & -0.09 & 0.17 & - \\
\hline 0 & 80 & $2220 \mathrm{C} 7$ & $18: 35: 30.72$ & $-34: 47: 42.70$ & 15.62 & 1.233 & 2.43 & 1.7 & 5200 & -0.05 & 0.14 & - \\
\hline 1 & 81 & $1554 \mathrm{C} 7$ & $18: 35: 40.37$ & $-34: 49: 16.14$ & 15.11 & 1.166 & 2.22 & 1.4 & 5200 & -0.39 & 0.14 & - \\
\hline 0 & 82 & $2178 \mathrm{C} 7$ & $18: 35: 41.56$ & $-34: 47: 48.85$ & 15.77 & 1.371 & 2.10 & 1.5 & 4500 & -0.02 & 0.17 & - \\
\hline 0 & 83 & $2422 \mathrm{C} 7$ & $18: 35: 48.06$ & $-34: 47: 15.63$ & 15.89 & 1.481 & 2.01 & 1.2 & 4300 & 0.26 & 0.25 & - \\
\hline 0 & 84 & $3101 \mathrm{C} 7$ & $18: 35: 37.13$ & $-34: 45: 34.59$ & 15.86 & 1.233 & 2.37 & 1.1 & 4900 & -0.34 & 0.14 & - \\
\hline 1 & 86 & 2580C6 & $18: 35: 15.24$ & $-34: 46: 41.38$ & 16.06 & 1.566 & 1.66 & 1.4 & 3850 & 0.42 & 0.27 & - \\
\hline 0 & 87 & $3238 \mathrm{C} 6$ & $18: 35: 16.99$ & $-34: 44: 59.64$ & 15.73 & 1.322 & 2.32 & 1.1 & 4900 & -0.17 & 0.15 & - \\
\hline 1 & 89 & $1417 \mathrm{C} 7$ & $18: 35: 26.03$ & $-34: 49: 34.46$ & 15.58 & 1.172 & 2.39 & 1.3 & 5150 & -0.31 & 0.24 & - \\
\hline 0 & 90 & $2532 \mathrm{C} 6$ & 18:35:13.18 & $-34: 46: 48.45$ & 15.71 & 1.345 & 2.17 & 1.4 & 4650 & -0.13 & 0.18 & - \\
\hline 0 & 91 & $2948 C 7$ & $18: 35: 24.53$ & $-34: 46: 01.16$ & 15.53 & 1.679 & 1.83 & 1.1 & 4250 & 0.02 & 0.17 & - \\
\hline 0 & 92 & 1589C7 & $18: 35: 30.62$ & $-34: 49: 10.88$ & 15.87 & 1.164 & 2.56 & 1.4 & 5250 & 0.11 & 0.18 & - \\
\hline 2 & 93 & $3690 \mathrm{C} 7$ & $18: 35: 28.35$ & $-34: 44: 08.73$ & 14.97 & 1.208 & 2.00 & 1.5 & 4900 & -1.40 & 0.18 & - \\
\hline 0 & 95 & 3796C6 & $18: 34: 58.25$ & $-34: 43: 44.19$ & 15.53 & 1.454 & 2.01 & 1.4 & 4500 & -0.83 & 0.13 & - \\
\hline 0 & 96 & 3786C6 & $18: 34: 49.35$ & $-34: 43: 45.09$ & 15.90 & 1.444 & 2.33 & 1.5 & 4800 & -0.28 & 0.21 & - \\
\hline 0 & 97 & $3201 \mathrm{C} 6$ & $18: 35: 03.02$ & $-34: 45: 05.51$ & 15.47 & 1.211 & 2.35 & 1.1 & 5150 & -0.05 & 0.16 & - \\
\hline 1 & 98 & $4217 \mathrm{C} 6$ & $18: 34: 55.12$ & $-34: 42: 46.34$ & 15.78 & 1.461 & 2.10 & 1.3 & 4500 & -0.39 & 0.15 & - \\
\hline 1 & 99 & $4333 \mathrm{C} 6$ & $18: 35: 04.91$ & $-34: 42: 31.72$ & 15.99 & 1.228 & 2.48 & 1.4 & 5000 & -0.16 & 0.20 & - \\
\hline 0 & 100 & 6164C6 & $18: 34: 58.84$ & $-34: 38: 17.52$ & 15.25 & 1.265 & 2.21 & 1.5 & 5050 & -0.17 & 0.14 & - \\
\hline 0 & 101 & $3350 \mathrm{C} 6$ & $18: 35: 14.53$ & $-34: 44: 44.95$ & 15.27 & 1.120 & 2.29 & 1.5 & 5200 & -0.19 & 0.17 & - \\
\hline 0 & 102 & $4263 \mathrm{C} 6$ & 18:35:01.81 & $-34: 42: 40.62$ & 15.87 & 1.329 & 2.43 & 1.3 & 5000 & -0.18 & 0.13 & - \\
\hline 0 & 103 & $3558 \mathrm{C} 6$ & 18:35:12.93 & $-34: 44: 17.26$ & 16.03 & 1.341 & 2.38 & 1.5 & 4800 & -0.02 & 0.15 & - \\
\hline 0 & 104 & 6090C6 & $18: 35: 20.05$ & $-34: 38: 28.85$ & 16.15 & 1.479 & 2.11 & 1.2 & 4300 & 0.11 & 0.22 & - \\
\hline 1 & 105 & 1419C6 & 18:34:59.16 & $-34: 49: 29.22$ & 15.82 & 1.485 & 2.12 & 1.2 & 4500 & 0.02 & 0.20 & - \\
\hline 1 & 106 & $5543 \mathrm{C} 6$ & $18: 35: 15.85$ & $-34: 39: 49.56$ & 16.07 & 1.459 & 2.08 & 1.3 & 4300 & 0.12 & 0.22 & - \\
\hline 0 & 107 & $5908 \mathrm{C} 6$ & $18: 34: 48.00$ & $-34: 38: 54.03$ & 15.66 & 1.151 & 2.41 & 1.3 & 5150 & -0.67 & 0.16 & - \\
\hline
\end{tabular}


M. Zoccali et al.: Galactic bulge iron abundance, Online Material $p 9$

Table 3. continued.

\begin{tabular}{|c|c|c|c|c|c|c|c|c|c|c|c|c|}
\hline $\mathrm{QF}^{a}$ & ID & OGLE-ID & RA & Dec & $V$ & $V-I$ & $\log g$ & $V_{\mathrm{t}}$ & $T_{\text {eff }}$ & {$[\mathrm{Fe} / \mathrm{H}]$} & $\sigma^{b}$ & Cluster? \\
\hline 0 & 108 & $4876 \mathrm{C} 6$ & $18: 35: 12.29$ & $-34: 41: 18.14$ & 16.08 & 1.303 & 2.46 & 1.3 & 4900 & -0.59 & 0.12 & - \\
\hline 0 & 109 & $5319 \mathrm{C} 6$ & $18: 34: 43.24$ & $-34: 40: 18.83$ & 15.58 & 1.447 & 2.09 & 1.3 & 4600 & -0.36 & 0.16 & _- \\
\hline 1 & 110 & $6263 \mathrm{C} 6$ & $18: 35: 14.21$ & $-34: 38: 05.96$ & 16.01 & 1.648 & 1.79 & 1.5 & 4000 & 0.64 & 0.38 & - \\
\hline 0 & 111 & 5977C6 & $18: 35: 12.22$ & $-34: 38: 45.31$ & 15.31 & 1.334 & 2.01 & 1.7 & 4650 & 0.09 & 0.17 & - \\
\hline 2 & 112 & $4612 \mathrm{C} 6$ & $18: 35: 01.15$ & $-34: 41: 54.14$ & 16.02 & 1.264 & 2.46 & 1.5 & 4950 & -0.82 & 0.17 & - \\
\hline 0 & 113 & $5588 \mathrm{C} 6$ & 18:35:06.42 & $-34: 39: 42.10$ & 15.87 & 1.282 & 2.37 & 1.1 & 4900 & -0.28 & 0.11 & - \\
\hline 0 & 114 & $5664 \mathrm{C} 6$ & 18:34:52.60 & $-34: 39: 31.75$ & 15.43 & 1.330 & 2.14 & 1.5 & 4800 & 0.15 & 0.19 & - \\
\hline 0 & 115 & 3965C6 & 18:34:59.18 & $-34: 43: 22.14$ & 15.46 & 1.663 & 1.91 & 1.5 & 4400 & -0.20 & 0.14 & - \\
\hline 0 & 116 & $159 \mathrm{C} 4$ & 18:34:30.32 & $-34: 36: 06.99$ & 15.66 & 1.435 & 2.18 & 1.5 & 4700 & -0.14 & 0.22 & - \\
\hline 0 & 117 & 6717C6 & 18:34:52.39 & $-34: 37: 01.77$ & 15.58 & 1.283 & 2.29 & 1.4 & 4950 & -0.23 & 0.13 & - \\
\hline 0 & 118 & 5980C6 & 18:34:43.98 & $-34: 38: 44.23$ & 15.93 & 1.242 & 2.45 & 1.4 & 5000 & -0.25 & 0.16 & - \\
\hline 0 & 119 & $7119 \mathrm{C} 5$ & $18: 34: 32.34$ & $-34: 37: 03.86$ & 15.12 & 1.381 & 1.96 & 1.3 & 4700 & -0.33 & 0.14 & - \\
\hline 0 & 120 & $6419 \mathrm{C} 5$ & 18:34:30.96 & $-34: 38: 29.97$ & 15.40 & 1.390 & 2.07 & 1.2 & 4700 & -0.25 & 0.17 & - \\
\hline 0 & 121 & $6230 \mathrm{C} 5$ & 18:34:33.98 & $-34: 38: 53.61$ & 15.06 & 1.154 & 2.18 & 1.3 & 5150 & 0.09 & 0.16 & - \\
\hline 2 & 122 & 6099C5 & $18: 34: 28.47$ & $-34: 39: 11.53$ & 15.72 & 1.288 & 2.34 & 1.3 & 4950 & -0.78 & 0.30 & - \\
\hline 2 & 123 & $5750 \mathrm{C} 5$ & $18: 34: 27.51$ & $-34: 39: 55.71$ & 15.56 & 1.130 & 2.35 & 1.4 & 5100 & -0.29 & 0.30 & - \\
\hline 0 & 124 & $5396 \mathrm{C} 5$ & 18:34:34.77 & $-34: 40: 42.70$ & 15.63 & 1.282 & 2.20 & 1.5 & 4750 & -0.16 & 0.22 & - \\
\hline 1 & 126 & $2502 \mathrm{C} 3$ & 18:34:54.42 & $-34: 30: 56.51$ & 15.97 & 1.330 & 2.44 & 1.4 & 4950 & -0.39 & 0.15 & - \\
\hline 0 & 128 & $1754 \mathrm{C} 3$ & 18:34:48.92 & $-34: 32: 32.69$ & 15.43 & 1.301 & 2.23 & 1.3 & 4950 & -0.20 & 0.12 & - \\
\hline 0 & 129 & $1407 \mathrm{C} 3$ & $18: 34: 50.53$ & $-34: 33: 22.68$ & 15.76 & 1.155 & 2.35 & 1.0 & 4950 & -0.82 & 0.20 & - \\
\hline 1 & 132 & $1700 \mathrm{C} 4$ & 18:34:29.77 & $-34: 32: 44.84$ & 15.67 & 1.288 & 2.35 & 1.4 & 5000 & 0.34 & 0.30 & - \\
\hline 1 & 135 & $166 \mathrm{C} 3$ & $18: 34: 45.39$ & $-34: 36: 07.17$ & 15.90 & 1.267 & 2.30 & 1.5 & 4750 & -0.86 & 0.24 & - \\
\hline
\end{tabular}

${ }^{a} \mathrm{QF}$ is a subjective quality factor, classifying stars into $\operatorname{good}(0)$, bad (2) and intermediate (1), according to how unique/degenerate the convergence into the final model atmosphere proceeded.

${ }^{b}$ Line-to-line dispersion around the mean $[\mathrm{Fe} / \mathrm{H}]$. 